\title{
On Recent Progress for the Stochastic Navier Stokes Equations
}

\author{
Jonathan C. Mattingly
}

\begin{abstract}
We give an overview of the ideas central to some recent developments in the ergodic theory of the stochastically forced Navier Stokes equations and other dissipative stochastic partial differential equations. Since our desire is to make the core ideas clear, we will mostly work with a specific example: the stochastically forced Navier Stokes equations. To further clarify ideas, we will also examine in detail a toy problem. A few general theorems are given. Spatial regularity, ergodicity, exponential mixing, coupling for a SPDE, and hypoellipticity are all discussed.
\end{abstract}

This article attempts to collect a number of ideas which have proven useful in the study of stochastically forced dissipative partial differential equations. The discussion will center around those of ergodicity but will also touch on the regularity of both solutions and transition densities. Since our desire is to make the core ideas clear, we will mostly work with a specific example: the stochastically forced Navier Stokes equations. To further clarify ideas, we will also examine in detail a toy problem. Though we have not tried to give any great generality, we also present a number of abstract results to help isolate what assumptions are used in which arguments. Though a few results are presented in new ways and a number of proofs are streamlined, the core ideas remain more or less the same as in the originally cited papers. We do improve sightly the exponential mixing results given in [Mat02c]; however, the techniques used are the same. Lastly, we do not claim to be exhaustive. This is not meant to be an all encompassing review article. The view point given here is a personal one; nonetheless, citations are given to good starting points for related works both by the author and others.

Consider the two-dimensional Navier-Stokes equation with stochastic forcing:

$$
\left\{\begin{array}{l}
\frac{\partial u}{\partial t}+(u \cdot \nabla) u+\nabla P=\nu \Delta u+\frac{\partial W(x, t)}{\partial t} \\
\nabla \cdot u=0
\end{array} .\right.
$$

MSC 2000 : 58G98 35K99.

Keywords : stochastic Navier Stokes, ergodicity, coupling, exponential mixing. hypoellipticity, SPDE, stochastic dissipative PDE 
We restrict to the $2 \pi$-periodic case with mean flow zero, though many of the results apply equally to bounded domains with Dirichlet boundary conditions. The addition of a stochastic forcing can be motivated by a number of considerations. Since the Navier-Stokes equations are dissipative, if there is no external forcing, the system relaxes to the zero state where the fluid is at rest. Hence, if one is interested in probing the nonlinear dynamics, some forcing is necessary. Stochastic forcing is often proposed, particularly in the study of turbulent fluid flows, as a way to add a "generic" forcing. Generic is then interpreted in the sense of the typical events in probability space.

We will take the forcing to be the sum of independent Brownian motions exciting independent Fourier modes. This is convenient because one of our long term goals is to understand the interaction between the different scales and the differences of the dynamics at different scales. Specifically we set

$$
W(x, t)=\sum_{k \in \mathcal{K}} \sigma_{k} \frac{k^{\perp}}{|k|} e^{i k \cdot x} \beta_{k}(t) \quad \text { with } \quad \sum_{k \in \mathcal{K}} \sigma_{k}^{2}<\infty
$$

where $\mathcal{K} \subset \mathbb{Z}^{2}$ does not contain the zero wave number ensuring that the spatial mean stays zero. The $\beta_{k}=\frac{1}{\sqrt{2}}\left(\beta_{k}^{(1)}+i \beta_{k}^{(2)}\right)$ where the $\beta_{k}^{(i)}$ are mean zero, variance one Brownian Motions independent except for the reality condition $\bar{\beta}_{k}=\beta_{-k}$. The $\sigma_{k} \in \mathbb{C}$ are constants used to set the spatial roughness of the flow. They also satisfy the reality condition $\bar{\sigma}_{k}=\sigma_{-k}$. We make the standing assumption that $\mathcal{E}_{0}=\sum\left|\sigma_{k}\right|^{2}<\infty$ and define $\sigma_{*}^{2}=\max \left|\sigma_{k}\right|^{2}$. Similarly if $\mathcal{E}_{\alpha}=\sum\left|\sigma_{k}\right||k|^{2 \alpha}<\infty$ then for every $t, W(\cdot, t)$ is almost surely in the Sobolev space $H^{\alpha}\left(\mathbb{T}^{2}\right) \times H^{\alpha}\left(\mathbb{T}^{2}\right)$. Here $\mathbb{T}^{2}$ is the two dimensional torus. If the $\left|\sigma_{k}\right|$ decay exponentially or faster, the forcing field is analytic is space almost surely.

In the next section, we continue with the setup. In section 2, we briefly discuss invariant measures. In section 3, we discuss how the structure of the solution changes for different choices of forcing. In particular, we discuss the spatial smoothness. In sections 4, 5, and 6, we highlight some of the difficulties with ergodic theory in infinite dimensions. In section 7, we discuss ergodicity of the stochastically forced Navier Stokes (SNS) equations under various assumptions, including the ideas of "effective ellipticity" and the reduction to Gibbsian dynamics (dynamics with memory). In section 8 , we formulate the results in a more general setting and examine a toy model to highlight the main ideas. In sections 9 and 10, we discuss the contractive nature of the SNS equations and the fluctuations of its energy and enstrophy. In section 13, we discuss the Lyapunov structure and localization in the general setting. In section 15, we prove a general exponential mixing result using a non-Markovian coupling argument. In section 16, we discuss some other systems where the discussed methods apply. In section 17, we give a number of partial results in the setting where the previously stated ergodic theorems do not hold. Lastly in section 18, we list a few open questions. 


\section{The Setting}

It is convenient to project (1) onto the space of divergence free vector fields thereby removing the pressure, which is just a Lagrange multiplier enforcing the divergence free constraint. To this end, $\mathbb{L}^{2}$ will denote the closure in the $L^{2}$ topology of divergence free, mean zero, $C^{\infty}$ vector fields on the two dimensional torus $\mathbb{T}^{2}$. Similarly the Sobolev space $\mathbb{H}^{\alpha}$ is defined as $\mathbb{L}^{2}$ except that the closure is taken in $H^{\alpha}\left(\mathbb{T}^{2}\right) \times H^{\alpha}\left(\mathbb{T}^{2}\right)$. Projecting equation (1) onto $\mathbb{L}^{2}$ produces the stochastic evolution equation

$$
\frac{\partial u(x, t)}{\partial t}+\nu \Lambda^{2} u(x, t)+B(u, u)=\frac{\partial W(x, t)}{\partial t}
$$

where $B(u, v)=P_{d i v}(u \cdot \nabla) v, \Lambda^{2} u=-P_{d i v} \Delta u$ and $P_{d i v}$ is the projection operator onto the space of divergence free vector fields.

To better elucidate some of the structure of (3), it is useful at times to consider the equation for the vorticity $\omega(x, t) \stackrel{\text { def }}{=} \frac{\partial u_{2}}{\partial x_{1}}-\frac{\partial u_{1}}{\partial x_{2}}$ written in Fourier Space. Notice that in two dimensions $\omega$ is a scalar quantity. Setting $\omega(x, t)=\sum_{k} \omega_{k}(t) e^{i k \cdot x}$, one obtains the infinite system of coupled diffusions

$$
\frac{d \omega_{k}(t)}{d t}=-\nu|k|^{2} \omega_{k}+i \sum_{\ell+j=k} \frac{k^{\perp} \cdot \ell}{|k|^{2}} \omega_{\ell} \omega_{j}+i|k| \sigma_{k} \mathbf{1}_{k \in \mathcal{K}} \frac{d \beta_{k}(t)}{d t}
$$

Unlike many lattices of interacting diffusions, this system in not invariant under translations in the lattice index $k \in \mathbb{Z}^{2}$. In fact for large $|k|$ the linear term in (4) dominates the other drift term which couples the modes together. This observation is at the heart of all that follows. It gives rise to the dissipative nature of the dynamics.

Since the noise is additive in our model problem, it is completely standard that there exists a stochastic flow which depends continuously on both the initial data and the noise realization $W$ considered as an element of the probability space $\Omega \stackrel{\text { def }}{=}$ $C\left((-\infty, \infty) ; \mathbb{R}^{2|\mathcal{K}|}\right)$. To complete the picture, we work on $\left(\Omega, \mathbb{P}, \mathcal{F}, \mathcal{F}_{t}\right)$. Here $\Omega$, as just defined, is the path space of the Brownian trajectories, $\mathbb{P}$ is the Weiner measure on this space, $\mathcal{F}$ is the associated sigma algebra, and $\mathcal{F}_{t}$ and $\mathcal{F}_{[s, t]}$ are the filtrations containing the information of the noise increments up to time $t$ and between time $s$ and $t$ respectively. We will at times write $\varphi_{s, t}(W) u_{0}$ or $\varphi_{s, t}^{W} u_{0}$ for $u(x, t, W)$ with $u(s)=u_{0}$ and $\varphi_{t}(W)$ for $\varphi_{0, t}(W)$. The notation $u_{[s, t]}$ means the segment of trajectory on $[s, t]$. We will write $\mathbb{E}$ to denote expectation with respect to the probability measure $\mathbb{P}$; that is $\mathbb{E} F(W)=\int_{\Omega} F(W) \mathbb{P}(d W)$. At times we will speak of solutions existing on the time interval $(-\infty, \infty)$. By this we mean a measure $P$ on $\Omega \times C(-\infty, \infty ; \mathbb{X})$ so that the following holds for almost every $(W, u)$ : $W$ is distributed as a Weiner process, $u(t)$ is adapted to the filtration generated by $W(s)$ with $s \leq t$, and the pair $(W, u)$ solves the integral form of (3) over any finite time interval. 


\section{Ergodicity and Invariant Measures}

When investigating a stochastically forced system, such as the stochastically forced Navier Stokes equation (SNS), the main interest is often the behavior and structure of the system once it has forgotten its initial condition. In other words, we are interested in the behavior of the system in its statistical steady state. The statistical steady states of a system are described by its invariant measure. In our setting, a measure $\mu$ on $\mathbb{L}^{2}$ is invariant under the dynamics if for any $t>0$ and Borel set $A \subset \mathbb{L}^{2}$

$$
\mu\left\{u_{0}: u_{0} \in A\right\}=\int_{\Omega} \mu\left\{u_{0}: u\left(t, W ; u_{0}\right) \in A\right\} \mathbb{P}(d W)=\mathbb{E} \mu\left\{u_{0}: \varphi_{t}(W) u_{0} \in A\right\} .
$$

A system is uniquely ergodic, or simply ergodic, if there is only one such invariant measure. The Birkoff ergodic theorem (cf. [Sin94]) guarantees that for any bounded function $f: \mathbb{L}^{2} \rightarrow \mathbb{R}$

$$
\frac{1}{T} \int_{0}^{T} f\left(u\left(t, W ; u_{0}\right)\right) d t \underset{T \rightarrow \infty}{\longrightarrow} \bar{f}\left(u_{0}\right)=\int_{\mathbb{L}^{2}} f(u) d \mu_{u_{0}}(u)
$$

if $u_{0}$ is a typical point for some invariant measure $\mu_{u_{0}}$. We have labeled the invariant measure with the initial point $u_{0}$ to emphasize that different points might converge to different $\bar{f}$, each the average of $f$ against a different invariant measure. However, if the system is ergodic then there is only one such invariant measure and the time average $\bar{f}$ is independent of the initial condition. Hence, the statistics of almost every trajectory will converge to a unique common distribution. Implying that the statistics of the systems asymptotic behavior is insensitive to the initial condition.

\section{The Form of the Forcing}

Consider the two classes of forcing distinguished by whether $|\mathcal{K}|<\infty$ or $|\mathcal{K}|=\infty$. The first class is the most natural from both the point of view of turbulence theory and that of exploring the nonlinear dynamics of the Navier-Stokes equations. In that case, one wants to force the equations at some scale, usually at large or intermediate scales, and then observe the transfer of energy and enstropy up and down scale. Generally, forcing which excites all the Fourier modes $\left(\mathcal{K}=\mathbb{Z}^{2}\right)$ is the first case studied for a given stochastic partial differential equation (SPDEs). This was true of the SNS (cf. [FM95, Fer97, DPZ96]). In these investigations, the forcing was assumed to be spatially rough; essentially $\left|\sigma_{k}\right| \sim|k|^{-\alpha}$. This assumption means that the forcing is not analytic in space. The requirement of rough forcing appears to not simply be a technical assumption, and the methods from [FM95, Fer97, DPZ96] do not seem to work in other elliptic cases. It is important to mention that the qualitative behavior of the system appears to be quite different depending on whether the magnitude of the modes decays at least exponentially or simply algebraically.

Consider the following two theorems proven respectively in [MS03] and [Mat02c]. The first theorem compares the vorticity equation to the associated linear stochastic 
heat equation. This equation is just the Ornstein-Uhlenbeck process

$$
\left\{\begin{array}{l}
\frac{\partial z}{\partial t}(t, x)=\nu \Delta z(t, x)+i \sum_{k}|k| \sigma_{k} \frac{\partial \beta_{k}(t)}{\partial t} \\
z(0, x)=\omega_{0}(x),
\end{array} .\right.
$$

If $z(x, t)=\sum_{k \in \mathbb{Z}} z_{k}(t) \exp (i k \cdot x)$ then $(5)$ becomes

$$
\frac{d z_{k}(t)}{d t}=-\nu|k|^{2} z_{k}+i|k| \sigma_{k} \frac{d \beta_{k}(t)}{d t} .
$$

The following theorem states that at small scales $z$ and $\omega$ are quite similar, even path wise if the forcing decays algebraically in the spatial Fourier modes.

Theorem 1. Assume that $c|k|^{-\alpha}<\left|\sigma_{k}\right|<C|k|^{-\alpha}$ for some positive constants. Let $\omega_{k}^{\prime}=\frac{\sqrt{2}}{\left|\sigma_{k}\right|} \omega_{k}$ and $z_{k}^{\prime}=\frac{\sqrt{2}}{\left|\sigma_{k}\right|} z_{k}$. For any uniformly continuous, bounded function $F$ on $C\left([0,1] ; R^{d}\right), E\left|F\left(\omega_{k_{1}}^{\prime}, \ldots, \omega_{k_{d}}^{\prime}\right)-F\left(z_{k_{1}}^{\prime}, \ldots, z_{k_{d}}^{\prime}\right)\right| \rightarrow 0$ as $k_{1}, \ldots, k_{d} \rightarrow \infty$. [MS03]

Theorem 1 says that when the forcing decays algebraically in the magnitude of the wave number $k$, then so does the solution. In fact, at small scales, it is pathwise a perturbation of (5) in some sense. Hence, the nonlinearity is really secondary in setting the infinite dimensional character of the problem.

The second theorem covers the case when the forcing decays at least exponentially fast and, in particular, covers the case when only a finite number of modes are forced. Earlier versions of this theorem were proven in [Mat98, MS99] and all of the versions build on deterministic versions which date back at least to [FT89] and are informed by later works such as [L097, DT95, DG95, OT00]. In [BKL00, Shi02], yet different formulations of Theorem 2 are given and proven. The second reference seems to give the best scaling with viscosity, while the version below gives explicit, eventually stationary processes which bound the quantities of interest.

Theorem 2. If there exist positive constants $\beta$ and $C$ so $\left|\sigma_{k}\right|<C e^{-\beta|k|}$ then for any initial $u(0) \in \mathbb{L}^{2}$ there exist two stochastic processes $\tau(t, W)$ and $D(t, W)$, positive for $t>0$, so that

$$
\left|u_{k}(t, W)\right| \leq D(t, W) e^{-\tau(t, W)|k|} W \text {-almost surely for all } t>0
$$

and such that $\lim _{t \rightarrow \infty} \mathbb{E} \tau(t) \in\left[c_{1}, C_{1}\right]$ and $\lim _{t \rightarrow \infty} \mathbb{E} D(t) \in\left[c_{2}, C_{2}\right]$ where $c_{i}$ and $C_{i}$ are positive constants which depend on the structure of the forcing but not on the initial data $u(0)$. (For the form of the equations for $\tau$ and $D$ and information about their moments see [Mat02b].)

Though no lower bound on $\left|u_{k}\right|$, as $|k| \rightarrow \infty$, has been proven, there is strong evidence that this is the correct order. Even when the forcing decays faster than exponential, there is no evidence that the solution does. It is interesting to note that in all of the current estimates of the decay rate fluctuate in time. Whether this is correct is not clear. It is a little surprising that even when only a few modes are forced that $\tau(t)$ does not converge to a constant as $t \rightarrow \infty$.

Comparing Theorem 1 and 2, one sees that there is a strong qualitative difference between the two cases. In the first, the forcing sets the small scale structure. In the second, the forcing seems to be dictated by the nonlinear dynamics. 


\section{The Difficulty of Infinite Dimensions}

It is reasonable to ask why the ergodic theory of stochastically forced PDEs is more complicated than that of finite dimensional SDEs. A basic problem is that there is no single distinguished topology associated with most infinite dimensional diffusions. Since all topologies are not equivalent, if one wants to write the transition density one must use exactly the right base measure. This means one must know exactly the natural topology of the problem. This is underlined by the following simple example. Consider two SPDEs of the form (5) with $\left|\sigma_{k}\right|=|k|^{-\alpha}$ in one case and $\left|\sigma_{k}\right|=|k|^{-\alpha+\epsilon}$ in the other. These two process induce measures on the phase space $L^{2}$ which are mutually singular at any positive moment of time, even if they start from the same point.

In general, getting the correct topology is a very delicate matter. There seems to be no good general tool to address this class of problems. In the setting of Theorem 1 , one strongly suspects that the measure induced by the SNS at a moment of time $t$ is equivalent to that induced by (5). However, even in this case, equivalence has only been proven when the Laplacian is replaced by $\Delta^{2+\epsilon}$. For this "hyperviscous" problem, the equivalence is proven in [MS03].

\section{Diffusions, Ellipticity, and Hypoellipticity}

Just as an ordinary SDE is associated with a PDE which evolves its density, one can association with an SPDE a "diffusion" on a larger space which evolves the probability transition density. In some cases this can be made rigorous (cf. [DPZ92, FG98, DPZ02], ). Formally, consider the "diffusion" on $\mathbb{R}^{Z^{2} \times Z^{2}}$ associated with the stochastic process (4). Writing $z_{k}=x_{k}+i y_{k}$, the backward Kolmogorov equation would be

$$
\begin{aligned}
\frac{\partial}{\partial t} U\left(\left\{x_{k}\right\},\left\{y_{k}\right\}, t\right) & =\mathcal{L} U\left(\left\{x_{k}\right\},\left\{y_{k}\right\}, t\right) \\
U\left(\left\{x_{k}\right\},\left\{y_{k}\right\}, 0\right) & =U_{0}\left(\left\{x_{k}\right\},\left\{y_{k}\right\}\right)
\end{aligned}
$$

where $U_{0}: \mathbb{R}^{Z^{2} \times Z^{2}} \rightarrow \mathbb{R}$ is the initial condition. By $\left\{x_{k}\right\}$ we mean the collection $\left\{x_{k}: k \in \mathbb{Z}^{2}\right\}$. The differential operator $\mathcal{L}$ is

$$
\mathcal{L}=\sum_{k} \operatorname{Re}\left(F_{k}\right) \frac{\partial}{\partial x_{k}}+\operatorname{Im}\left(F_{k}\right) \frac{\partial}{\partial y_{k}}+\frac{1}{2} \mathbf{1}_{k \in \mathcal{K}}|k|^{2}\left|\sigma_{k}\right|^{2}\left(\frac{\partial^{2}}{\partial x_{k}^{2}}+\frac{\partial^{2}}{\partial y_{k}^{2}}\right)
$$

where

$$
F_{k}=-\nu|k|^{2} z_{k}+i \sum_{\ell+j=k} \frac{k^{\perp} \cdot \ell}{|k|^{2}} z_{\ell} z_{j}
$$

The case when $\mathcal{K}=\mathbb{Z}^{2}$, corresponds to the elliptic setting. If $|\mathcal{K}|<\infty$, then the operator $\mathcal{L}$ is degenerate to leading order in all but a finite number of coordinates. Even the case $\mathcal{K} \neq \mathbb{Z}^{2}$ but $|\mathcal{K}|=\infty$, it is still degenerate. In either of the last cases, the ergodic theorems stated previously are surprising in the sense that they imply some sort of ellipticity without requiring the detailed geometric information needed to verify hypoellipticity. These ideas will be elaborated upon in section 17 . 


\section{Ergodicity with Elliptic, Rough Forcing}

In [FM95, Fer97] ergodicity is proven under the assumption, translated to our setting, that $c|k|^{-\alpha}<\left|\sigma_{k}\right|<C|k|^{-\alpha}$ for some positive constants. The proof of ergodicity relies on the Bismuth-Elworthy-Li formula and seems to fundamentally require an elliptic diffusion with algebraically decaying spectrum. In light of Theorem 1, it is tempting to characterize the system in this regime as a perturbation of the linear process since the linear process sets the small scale structure. Eckmann and Hairer [EH01] showed that finite dimensional Malliavin calculus could be combined with the type of analysis used in [Cer99, FM95, Fer97] to show that a stochastically forced SPDE was ergodic even if a finite number of the directions with possibly positive Lyapunov exponents were not forced. They required a bracket condition in the spirit of Höromander's "sum of squares theorem" (see section 17). Unfortunately they still required rough (algebraically decaying) forcing.

\section{Ergodicity under an Effective Ellipticity Assumption}

We now turn to a number of results which allow one to prove ergodicity despite the fact that $\mathcal{K} \neq \mathbb{Z}^{2}$. In particular, no lower bound will be placed on the decay rate of the $\left|\sigma_{k}\right|$; even $|\mathcal{K}|<\infty$ will be allowed if other assumptions are satisfied. Recalling that $\mathcal{E}_{0}=\sum_{k}\left|\sigma_{k}\right|^{2}$, we have the following theorem.

Theorem 3. There exists a fixed constant $\mathcal{C}$ depending only on the domain so that the following hold:

- If $\mathcal{C} \frac{\mathcal{E}_{0}}{\nu^{3}}<1$ then (3) has a unique $\mathbb{L}^{2}$-valued invariant probability measure regardless of the structure of the forcing. [Mat98, Mat99]

- If $\left|\sigma_{k}\right|>0$ for all $k$ with $|k|^{2} \in\left(0, \mathcal{C} \frac{\mathcal{E}_{0}}{\nu^{3}}\right)$, then (3) has a unique $\mathbb{L}^{2}$-valued invariant probability measure. [EMSO1, BKL01]

By a $\mathbb{L}^{2}$-valued probability measure, we mean a measure $\mu$ such that $\mu\left(\mathbb{L}^{2}\right)=1$. The existence was given in [VF88, Fla94] in the case of the SNS and in a more general setting in [CK97]. Both results of Theorem 3 stem from the following fact first proven in the stochastic setting in [Mat98] but closely related to ideas in [FP67, Tem95, CFNT89, FST88]. Contemporaneously to [EMS01] similar techniques were used in [KS00], to prove a similar theorem for impulsive or "kicked" forcing. Though these initial results applied only for bounded forced, those authors later extended them to cover unbounded forcing. They also proved a convergence theorem of the kicked case to the white in time case. For the remainder of the discussion of the SNS, we fix a positive $N_{*}$. Let $\Pi_{\ell}$ be the orthogonal projection onto the space spanned by the wave numbers $k$ with $|k|<N$ and let $\Pi_{h}$ be the complimentary orthogonal projection. We consider the "high mode" equation on $\Pi_{h} \mathbb{L}^{2}$ given by

$$
\frac{\partial h(x, t)}{\partial t}+\nu \Lambda^{2} h(x, t)+\Pi_{h} B(h+\ell, h+\ell)=\frac{\partial \eta(x, t)}{\partial t}
$$

where $\ell$ is a given "low mode" trajectory in $\Pi_{\ell} \mathbb{L}^{2}$ and $\eta=\Pi_{h} W(x, t)$. We will denote by $\Phi_{s, t}^{\eta}\left(\ell_{[s, t]} ; h_{0}\right)$ the solution to $(8)$ at time $t$ with initial condition $h_{0}$ at 
time $s$ and the given external forcings $\ell$ and $\eta$ over the time interval $[s, t]$. A more quantitative version of the following result is given in Lemma 13.1.

Theorem 4 (Foias and Prodi'67, Mattingly'98). Let $\mathcal{C}$ be the same constant as in Theorem 3. Assuming that $N_{*}^{2} \geq \mathcal{C} \frac{\mathcal{E}_{0}}{\nu^{3}}$, there exists a positive constant $\gamma$ so the following two statements hold.

- Let $u(x, t, W)$ be a solution to (3) on the time interval $[0, \infty)$. Define $\ell(t)=$ $\Pi_{\ell} u(t)$ and $\eta(t)=\Pi_{h} W(t)$. For almost every $W$, there exists a positive constant $T=T(W, u(0))$ so that for all $t \geq T$ and $h_{0} \in \Pi_{h} \mathbb{L}^{2}$

$$
\left|\Phi_{0, t}^{\eta}\left(\ell_{[0, t]} ; h_{0}\right)-\Pi_{h} u(t, W)\right|_{\mathbb{L}^{2}} \leq\left|h_{0}-\Pi_{h} u(0)\right|_{\mathbb{L}^{2}} e^{-\gamma t} .
$$

In particular, if $\tilde{u}(x, t, W)$ is another solution on $[0, \infty)$ and $\Omega_{0} \subset \Omega \times \Omega$ such that for all $(W, \tilde{W}) \in \Omega_{0}$ and $t \in[0, \infty)$ one has $\Pi_{\ell} \tilde{u}(t, W)=\Pi_{\ell} u(t, \tilde{W})$ and $\Pi_{h} W(t)-\Pi_{h} W(0)=\Pi_{h} \tilde{W}(t)-\Pi_{h} \tilde{W}(0)$ then $\tilde{u}(t, W)=u(t, \tilde{W})$ for all $t \in[0, \infty)$ and almost every $(W, \tilde{W}) \in \Omega_{0}$.

- Let $u(x, t, W)$ be a stationary solution to (3) on the time interval $(-\infty, \infty)$. Define $\ell(t, W)=\Pi_{\ell} u(t, W)$ and $\eta(t)=\Pi_{h} W(t)$. Then with probability one, there exists a positive constant $C$ depending only the solution $u$ so that for $t \leq 0$

$$
\left|\Phi_{t, 0}^{\eta}\left(\ell_{[t, 0]} ; h_{0}\right)-\Pi_{h} u(0)\right|_{\mathbb{L}^{2}} \leq C\left(\left|h_{0}\right|_{\mathbb{L}^{2}}+1\right) e^{-\gamma|t|}
$$

In particular, if $\tilde{u}(x, t, W)$ is another stationary solution on $(-\infty, \infty), \Omega_{0} \subset$ $\Omega \times \Omega$, and $T$ a fixed time, such that for any $(W, \tilde{W}) \in \Omega_{0}$ and $s \in(-\infty, T]$, $\Pi_{\ell} \tilde{u}(s, \tilde{W})=\Pi_{\ell} u(s, W)$ and $\Pi_{h} W(s)-\Pi_{h} W(0)=\Pi_{h} \tilde{W}(s)-\Pi_{h} \tilde{W}(0)$ then $u(s, W)=\tilde{u}(s, \tilde{W})$ for all $s \in(-\infty, T)$ and almost every $(W, \tilde{W}) \in \Omega_{0}$.

In other words, the history of the modes with wave number $|k|$ less than $N_{*}$ combined with the history of the forcing increments on the remaining degrees of freedom is sufficient to determine the solution uniquely with probability one.

The first statement in Theorem 3 is really a consequence of the contractive properties used to prove Theorem 4 . It is the special case when the set of determining low modes is empty; hence, knowledge of the infinite past of the random forces is sufficient to reconstruct the state of the whole system. In general, as shown in Theorem 4, one needs some finite number of determining modes and knowledge of the random forcing applied to the missing modes to reconstruct the missing modes.

We now give a more general result which implies the first part of Theorem 3 by showing that to each realization of noise there corresponds a unique, stationary solution if the viscosity is large enough relative to the forcing. Another way of saying this is that the system's random attractor, whose existence was proven at any viscosity by Flandoli [Fla94], consists of a trivial diffusing point. Schmalfuss proved a similar statement using a random fixed point argument in the case of multiplicative noise and large viscosity [Sch97]. In that case, the attracting random solution is a random fixed point which does not fluctuate in time. 
Theorem 5. If $\mathcal{C} \frac{\mathcal{E}_{0}}{\nu^{3}}<1$, then there exists a unique stationary random solution $u^{*}(t, W)$ defined for $t \in(-\infty, \infty)$ and almost all $W \in \Omega$. In addition, it attracts all other solutions exponentially quickly. [Mat98, Mat99]

One of the interesting interpretations of Theorem 4 in the case of arbitrary viscosity is that on the set of stationary solutions one can define a functional $\Phi$ : $C\left(-\infty, 0 ; \Pi_{\ell} \mathbb{L}^{2}\right) \rightarrow \Pi_{h} \mathbb{L}^{2}$ which reconstructs the high modes from the low modes. In particular if $u$ is a stationary solution and $\eta=\Pi_{h} W$ then define

$$
\Phi^{\eta}\left(\Pi_{\ell} u_{(-\infty, 0]}\right) \stackrel{\text { def }}{=} \lim _{t \rightarrow-\infty} \Phi_{t, 0}^{\eta}\left(\Pi_{\ell} u_{(t, 0]} ; h_{0}\right)
$$

for some arbitrary fixed $h_{0}$. Theorem 4 guarantees that the limit exists, that it is independent of the choice of $h_{0}$, and that $\Pi_{h} u(0, W)=\Phi^{\eta}\left(\Pi_{\ell} u_{(-\infty, 0]}\right)$. With this result, we can close the low mode equations at the price of introducing memory. One obtains

$$
\frac{d \ell(x, t)}{d t}+\nu \Lambda^{2} \ell(x, t)+\Pi_{\ell} B\left(\ell+\Phi^{\theta_{t} \eta}\left(\theta_{t} \ell\right), \ell+\Phi^{\theta_{t} \eta}\left(\theta_{t} \ell\right)\right)=\frac{\partial \xi(x, t)}{\partial t}
$$

where $\theta_{t}$ is the shift defined on $\ell$ by $\left(\theta_{t} \ell\right)(s)=\ell(s+t)$ and $\eta$ by $\left(\theta_{t} \eta\right)(s)=\eta(t+$ $s)-\eta(t)$. This representation is closely related and inspired by the inertial form representation from inertial manifolds theory (cf. [CFNT89, EFNT94]) and the ideas of symbolic dynamics. From the representation in (9), it is clear why it might be reasonable to call systems satisfying the assumptions of the second part of Theorem 3 "effectively elliptic" diffusions. Under that assumption, the system reduces to an equation of the form (9). This Itô process with memory is elliptic in the sense that the noise directly agitates all of the coordinates. In contrast to the hypoelliptic systems considered in section 17, no detailed knowledge of the tangent space structure is needed. Once the assumption about all of the possibly unstable directions being forced is satisfied, only some soft general estimates are needed.

When viewed in the context of Section 5, Theorem 3 might seem surprising. The theorem allows the associated diffusion to be degenerate in an infinite number of directions; yet the system has nice ergodic properties. Yet in other ways, Theorem 3 is expected. It simply says that if all of the unstable directions are forced directly, the system is ergodic. Since the long time dynamics are governed by the behavior on the "unstable manifold" (if one was known to exist), forcing those directions destroys all possible obstruction to mixing in the phase space. Since these systems are non-autonomous, when we say that a collection of directions are stable, we really mean that all of the associated Lyapunov exponents associated with these degrees of freedom are negative.

\section{Ergodicity: General Constructions}

We now lay out a more general framework to make some ideas clear without being encumbered by specifics. In the next section, we also give a simple toy model and some illustrative examples which hopefully will make the ideas concrete. 
Let $\left(\mathbb{X},|\cdot|_{\mathbb{X}},\langle\cdot, \cdot\rangle_{\mathbb{X}}\right)$ be a complete separable Hilbert space with a basis $\left\{e_{k}\right\}$, $k=1, \ldots$. Consider the stochastic evolution equation

$$
\frac{d u}{d t}=G(u)+\frac{d W}{d t} .
$$

taking values in $\mathbb{X}$. For concreteness, we take $W(x, t)=\sum \sigma_{k} e_{k} b_{k}(t)$ where $\sigma_{k}$ are constants which fix the structure of the forcing and the $b_{k}(t)$ are standard variance one Brownian motions. More general forcings built over a cylindrical Wiener space are possible with further assumptions, but this will be sufficient for our needs.

We assume that the $\sigma_{k}$ are chosen so that (10) has a globally defined stochastic flow $\varphi_{s, t}^{W} u_{0}=u(t, W)$ where $u(s)=u_{0}$. It is standard to associate with this flow a random dynamical system defined by the skew flow $\Theta_{t}(u, W)=\left(\varphi_{0, t}^{W} u_{0}, \theta_{t} W\right)$ (cf. [Arn98, Kif86]). Here $\theta_{t}$ is the shift operator. On noise paths the shift is defined by $\left(\theta_{t} W\right)(s)=W(t+s)-W(t)$. We also define the shift of a trajectory by $\left(\theta_{t} u\right)(s)=u(t+s)$. The difference in definition is due to the fact that in the first case we are really shifting the noise increments and not the path itself.

Fix a positive integer $N_{*}$, and define the splitting of the space $\mathbb{X}=\mathbb{X}_{\ell} \times \mathbb{X}_{h}$, by $\mathbb{X}_{\ell}=\operatorname{span}\left\{e_{k}: k<N_{*}\right\}$ and $\mathbb{X}_{h}=\operatorname{span}\left\{e_{k}: k \geq N_{*}\right\}$. Let $\Pi_{\ell}$ and $\Pi_{h}$ be the orthogonal projectors onto $\mathbb{X}_{\ell}$ and $\mathbb{X}_{h}$ respectively. We will write $u=(\ell, h)=$ $\left(\Pi_{\ell} u, \Pi_{h} u\right) \in \mathbb{X}_{\ell} \times \mathbb{X}_{h}$ and $\eta=\Pi_{h} W$ and $\xi=\Pi_{\ell} W$. Notice that the probability measure $\mathbb{P}$ decomposes into $\mathbb{P}_{\eta} \times \mathbb{P}_{\xi}$. As before, we will denote segments of trajectories by an interval of time as a subscript. Hence, $\ell_{[s, t]}$ is a trajectory in $\mathbb{X}_{\ell}$ between time $s$ and $t$. We use $\Pi_{[s, t]}$ to denote the projection of a path or set of paths onto the time interval $[s, t]$.

One can always split the system into two coupled equations on $\mathbb{X}_{\ell} \times \mathbb{X}_{h}$,

$$
\begin{aligned}
& \frac{d h}{d t}=\Pi_{h} G(\ell+h)+\frac{d \eta}{d t} \\
& \frac{d \ell}{d t}=\Pi_{\ell} G(\ell+h)+\frac{d \xi}{d t} .
\end{aligned}
$$

As in section 8.1, given this splitting, one can always define a map $h(t)=\Phi_{s, t}^{\eta}\left(\ell_{[s, t]} ; h_{0}\right)$ which solves (11) given an initial condition $h_{0}$, noise path $\eta$, and $\ell_{[s, t]}$ viewed as an external input. Then for each $t_{0}, h_{0}$, and $\eta$, we can define

$$
\begin{aligned}
\frac{d \ell}{d t} & =\Pi_{\ell} G\left(\ell+\Phi_{t_{0}, t}^{\eta}\left(\ell_{\left[t_{0}, t\right]} ; h_{0}\right)\right)+\frac{d \xi}{d t} \\
\ell\left(t_{0}\right) & =\ell_{0} .
\end{aligned}
$$

Equation (13) is no longer a standard diffusion as we have introduced memory through the function $\Phi_{t_{0}, t}^{\eta}$. It is critical to notice that $\ell(t)$ remains an adapted Itô process and hence the power of stochastic calculus can be brought to bear.

For the representation in (13) to be useful in the study of the ergodic theory of (10), the reduced dynamics (13) must "forget" the choice of $h_{0}$. One way to investigate this is to study the system as $t_{0} \rightarrow-\infty$. If the functional $\Phi$ becomes independent of $h_{0}$, then we have a closed dynamics on $C\left(-\infty, 0 ; \mathbb{X}_{\ell}\right)$ over the probability space $\Omega$. The resulting stochastic process could have infinite memory. Since it is 
defined by a compatible family of Gibbs measures, in [EMS01] it was dubbed "Gibbsian dynamics" to be contrasted with Markovian dynamics. The ergodic theory of systems with this type of memory was explored in its own right in [Bak02, BM03].

Alternatively, one could study the measures induced on the infinite future for different choices of $h_{0}$ and show that they induce the same asymptotic dynamics in some sense. This was the point of view taken in [Mat02c].

The two approaches are more or less equivalent and each has its own difficulties. One difficulty of the memory/Gibbsian Dynamics approach is that sometimes the limit, $\lim _{t_{0} \rightarrow-\infty} \Phi_{t_{0}, t}^{\eta}\left(\ell_{\left[t_{0}, t\right]} ; h_{0}\right)$, only exists on a restricted set of paths. In any situation where the approach works, one can always take $\ell_{(-\infty, t]}$, which are typical realizations of a stationary solution obtained by suspending any invariant measure over path space. But such a characterization is not constructive and at times is difficult to work with.

At the most basic level, the success of the approach developed in [EMS01] (or [KS00, BKL01] for that mater) hinges on treating the $\ell$ and $h$ variables in fundamentally differently way. Since the $\ell$ variable is finite dimensional in all the situations we consider, all the difficulties of probabilistic calculations in an infinite dimensional setting, mentioned in section 4 , are not an issue. In particular, the time $t$ transition densities projected onto $\mathbb{X}_{\ell}$ will have densities relative to Lebesgue measure on $\mathbb{X}_{\ell}$ if all of the directions in $\mathbb{X}_{\ell}$ are forced. The analysis of the $h$ variable is dynamic in nature. The analysis is done noise realization by noise realization. In contrast the analysis of the $\ell$ variable is probabilistic in nature. Arguments are made at the level of transition densities. If the system is strongly contractive, then the structure of the forcing is irrelevant. This was the fundamental fact used in [Mat99] to prove ergodicity by showing the existence of a distinguished globally attracting solution. Another way to say this is that the random attractor is trivial, consisting of a single point at each moment of time. Given our splitting, a similar structure remains in the $h$ variable. As we will see, such contraction, $\eta$-fiber by $\eta$-fiber, is much less sensitive to the topology than are questions like the absolute continuity of measures. The basic idea is to change the measure on the $\ell$ variables in such a way that the remaining degrees of freedom are contractive. The analyses in [Mat02c] and [EMS01] accomplish this by making the $\ell$ 's agree after some finite time. In [Hai02], the measure is changed to bring the $\ell$ (and $h$ ) together asymptotically at infinity but never at a finite time. In all cases, care must be taken so that the changes in the measure to not accumulate to the extent that the limiting measures become singular.

To execute this program, we need to analyze the dynamics on the path space of $\mathbb{X}_{h}$ and understand the structure of the measures induced on the path space of $\mathbb{X}_{\ell}$. To this end, we make a few definitions. For all $t>s \geq 0$ define

$$
\begin{aligned}
Q_{t}\left(\ell_{0}, h_{0}, A\right) & =\mathbb{P}\left(\ell(t) \in A \mid \ell(0)=\ell_{0}, h(0)=h_{0}\right) \\
Q_{[s, t)}\left(\ell_{0}, h_{0}, B\right) & =\mathbb{P}\left(\ell_{[s, t)} \in B \mid \ell(0)=\ell_{0}, h(0)=h_{0}\right)
\end{aligned}
$$

for Borel sets $A \subset \mathbb{X}_{\ell}$ and $B \subset C\left([s, t) ; \mathbb{X}_{\ell}\right) \cong C\left([0, t-s) ; \mathbb{X}_{\ell}\right)$. Notice we have associated $C\left([s, t), \mathbb{X}_{\ell}\right)$ with $C\left([0, t-s), \mathbb{X}_{\ell}\right)$ and will view $u_{[s, t)}$ as an element of $C\left([0, t-s), \mathbb{X}_{\ell}\right)$. 
Similarly for any realization of $\eta$, let $\mathcal{F}_{[s, t]}^{\eta}$ be the $\sigma$-algebra generated by the increments of $\eta$ between $[s, t]$. We define $Q_{t}^{\eta}\left(\ell_{0}, h_{0}, A\right)=\mathbb{P}\left(\ell(t) \in A \mid \ell(0)=\ell_{0}, h(0)=\right.$ $\left.h_{0}, \mathcal{F}_{[0, t]}^{\eta}\right)$ and $Q_{[s, t)}^{\eta}\left(\ell_{0}, h_{0}, B\right)=\mathbb{P}\left(\ell_{[s, t)} \in B \mid \ell(0)=\ell_{0}, h(0)=h_{0}, \mathcal{F}_{[s, t]}^{\eta}\right)$. These are analogous to the previous measures except that we have conditioned on the realization of $\eta$ over the time interval in question. Hence, for $A \subset \mathbb{X}_{\ell}$.

$$
Q_{t}\left(\ell_{0}, h_{0}, A\right)=\int Q_{t}^{\eta}\left(\ell_{0}, h_{0}, A\right) \mathbb{P}(d \eta)=\mathbb{E} Q_{t}^{\eta}\left(\ell_{0}, h_{0}, A\right) .
$$

\subsection{A Toy Problem}

We now describe a simple toy problem which contains the main ideas needed to prove the results of the previous section. We will use the same notation to make the connections explicit.

Consider the following two dimensional stochastic differential equation

$$
\begin{aligned}
\frac{d h(t)}{d t} & =-\nu_{1} h+F_{1}(\ell, h)+\sigma_{1} \frac{d \eta}{d t} \\
\frac{d \ell(t)}{d t} & =-\nu_{2} \ell+F_{2}(\ell, h)+\sigma_{2} \frac{d \xi}{d t} .
\end{aligned}
$$

Here $\nu_{i}>0, \sigma_{i} \geq 0, \eta$ and $\xi$ are standard one dimensional Brownian Motions on the probability space $\Omega=C\left((-\infty, \infty) ; \mathbb{R}^{2}\right)$. Hence, in the notation of the previous section $\mathbb{X}=\mathbb{R}^{2}, \mathbb{X}_{\ell}=\mathbb{R}$, and $\mathbb{X}_{h}=\mathbb{R}$. We assume the following estimates hold $\left|F_{1}\right|+\left|F_{2}\right|<K$ and $\left|F_{i}(\ell, h)-F_{i}(\ell, \tilde{h})\right| \leq L_{i}|h-\tilde{h}|$. For the moment, we allow either or both of the $\sigma_{i}$ to be zero. Eventually, we will require only that $\sigma_{2}>0$ allowing $\sigma_{1}$ to be zero if desired. Since the $F_{i}$ are uniformly bounded, it is easy to see that $\lim \sup _{t \rightarrow \infty} \mathbb{E}\left[h^{2}(t)+\ell^{2}(t)\right]$ is uniformly bounded over all initial conditions. From this, one can deduce the existence of an invariant measure using standard tightness arguments. The stochastic flow $\varphi_{t}^{(\xi, \eta)}\left(\ell_{0}, h_{0}\right)$ and the functional $\Phi_{s, t}^{\eta}\left(\ell_{[s, t]} ; h_{0}\right)$ are defined as in the previous section.

Subtracting two copies of (15) with the same $\eta$ and $\ell_{[s, t]}$ but different initial conditions produces the estimate

$$
\left|\Phi_{s, t}^{\eta}\left(\ell_{[s, t]} ; h_{0}\right)-\Phi_{s, t}^{\eta}\left(\ell_{[s, t]} ; \tilde{h}_{0}\right)\right| \leq\left|h_{0}-\tilde{h}_{0}\right| e^{-\left(\nu_{1}-L_{1}\right)(t-s)} .
$$

Using this estimate immediately produces the following result, which is the analog of Theorem 4.

Lemma 8.1. Assume $\nu_{1}>L_{1}$. Given $\ell \in C((-\infty, 0] ; \mathbb{R})$ and $h_{0} \in \mathbb{R}$, the limit

$$
\Phi^{\eta}\left(\ell_{(-\infty, 0]}\right) \stackrel{\text { def }}{=} \lim _{s \rightarrow-\infty} \Phi_{s, 0}^{\eta}\left(\ell_{[s, 0]} ; h_{0}\right)
$$

is well defined almost surely and independent of $h_{0}$. Similarly, fixing a time interval $[s, t]$ and initial conditions $\left(\ell_{i}(s), h_{i}(s)\right)$. Let $\Omega_{0} \subset \Omega \times \Omega$ such that for all $\left(\xi_{1}, \eta_{1}, \xi_{2}, \eta_{2}\right) \in \Omega_{0} \eta_{1}(r)-\eta_{1}(s)=\eta_{2}(r)-\eta_{2}(s)$ and $\ell_{1}(r)=\ell_{2}(r)$ when $r \in[s, t]$ where $\left(\ell_{i}(r), h_{i}(r)\right)=\varphi_{t}^{\left(\xi_{i}, \eta_{i}\right)}\left(\ell_{i}(s), h_{i}(s)\right)$. Then for all $\left(\xi_{1}, \eta_{1}, \xi_{2}, \eta_{2}\right) \in \Omega_{0}$

$$
\left|h_{1}(t)-h_{2}(t)\right| \leq\left|h_{1}(s)-h_{2}(s)\right| e^{-\left(\nu_{1}-L_{1}\right)(t-s)} .
$$


Recalling that the shift $\theta_{t}$ on trajectories acts by $\left(\theta_{t} \ell\right)(s)=\ell(t+s)$ and on noise paths by $\left(\theta_{t} \eta\right)(s)=\eta(t+s)-\eta(t)$, then by Lemma 8.1 we can reduce the system to the following system with memory

$$
\begin{aligned}
\frac{d \ell}{d t} & =-\nu_{2} \ell+F_{2}\left(\ell, \Phi^{\theta_{t} \eta}\left(\theta_{t} \ell\right)\right)+\sigma_{2} \frac{d \xi}{d t} \\
\ell(0) & =\ell_{0}
\end{aligned}
$$

where now $\ell(t)$ is seen as an element of $C((-\infty, t] ; \mathbb{R})$. Similarly our initial condition $\ell_{0}$ is an element of $C((-\infty, 0] ; \mathbb{R})$.

We now turn to another auxiliary result which, along with the contraction embodied Lemma 8.1, is the linchpin on which ergodicity hangs. Recalling the definitions from (14), we have

Lemma 8.2. Assume $\sigma_{2}>0$ and $\nu_{1}>L_{1}$. For all $\ell_{0}, h_{0} \in \mathbb{R}$, the measure $Q_{t}\left(\ell_{0}, h_{0}, \cdot\right)$ is equivalent to Lebesgue measure. For all $\ell_{0}, h_{0}, \tilde{h}_{0}, \in \mathbb{R}$, the measure $Q_{[0, \infty)}\left(\ell_{0}, h_{0}, \cdot\right)$ is equivalent to $Q_{[0, \infty)}\left(\ell_{0}, \tilde{h}_{0}, \cdot\right)$. For any realization of $\eta$, the exact same conclusions hold with $Q_{t}$ replace by $Q_{t}^{\eta}$ and $Q_{[0, \infty)}$ replaced by $Q_{[0, \infty)}^{\eta}$.

In the next section, we will use the Lemma 8.2 to prove the ergodicity of the toy problem (equations (15) and (16)). Of course, if $\sigma_{1}, \sigma_{2}>0$ then the system is uniformly elliptic and the fact that there is a unique invariant measure follows from standard elliptic theory. Even when $\sigma_{1}=0$, one might well use hypoelliptic diffusion theory to prove ergodicity. What we present here is a different possible route, where the detailed knowledge of the tangent space structure used in hypoelliptic arguments is replaced with assumptions about the system's Lyapunov exponents. The advantage of this route being that the contractive properties are less sensitive to the choice of topology than the measure theoretic properties of the system needed for the more standard approaches to ergodicity.

The fact that $Q_{[0, \infty)}\left(\ell_{0}, h_{0}, \cdot\right)$ and $Q_{[0, \infty)}\left(\ell_{0}, \tilde{h}_{0}, \cdot\right)$ are equivalent measures on the infinite time $[0, \infty)$ interval is critical. Absolute continuity on finite time intervals would not be sufficient. As an illustrative example consider the measures induced on path space by a standard Brownian motion $B(t)$ and the SDEs

$$
\begin{array}{rlrl}
\frac{d X(t)}{d t} & =-X(t)+\frac{d B(t)}{d t} & \frac{d Y(t)}{d t} & =-Y(t)+\frac{1}{t}+\frac{d B(t)}{d t} \\
X(0) & =x_{0} & Y(0) & =x_{0} .
\end{array}
$$

All three processes induce measures which are pairwise equivalent on any finite segment of path space. However, only the processes $X$ and $Y$ are equivalent on the infinite futures because their difference, $\frac{1}{t}$, is square integrable on an infinite time interval. See the proof of the second part Lemma 8.2 for the needed argument. In particular, we see that $X(t)$ and $Y(t)$ have the same asymptotic behavior at the level of the path space marginals, while $W(t)$ has a different one. Notice that we do not mean that $|X(t)-Y(t)| \rightarrow 0$ as $t \rightarrow \infty$.

Intuitively it is clear why Lemma 8.2 when combined with the contractive estimate from (17), implies that there is only one invariant measure. From Lemma 8.2 , we see that any two invariant measures will induce equivalent measure in 
$C\left(0, \infty ; \mathbb{X}_{\ell}\right)$. Hence they will charge trajectories with the same projection onto $\mathbb{X}_{\ell}$. This is already enough to ensure that the distribution on $\mathbb{X}_{\ell}$ is unique. However because of (17) if the two paths share the same projection on to $\mathbb{X}_{\ell}$ for all time the remaining degrees of freedom will also converge. Hence the time averages along some typical paths of the two measure will be the same. This implies the measure are the same. In Theorem 6, we make this argument precise.

PROOF OF THE FIRST PART OF LEMma 8.2: We now prove the statements concerning $Q_{t}$ and $Q_{t}^{\eta}$. We need only to show that the measures, conditional on $\eta$, are equivalent since the full measures are simply the integration of the conditioned measures against the Wiener measure governing $\eta$. We will use Girsanov's Theorem (cf. [Oks92, RY94] Ch 8, Thm 1.1) to compare (16) with the OrnsteinUhlenbeck process $\frac{d z}{d t}=-\nu_{2} z+\sigma_{2} \frac{d \xi}{d t}$. Girsanov's Theorem states that the two measures on path space are equivalent if a certain exponential martingale, which gives the Radon-Nikodym derivative, is uniformly integrable. This is guaranteed by Novikov's criterion (cf. [Oks92, RY94] Ch 8, Prop 1.15) which, translated into our setting, becomes $\mathbb{E} \exp \left(\frac{1}{2} \int_{0}^{t} \frac{1}{\sigma_{2}^{2}}\left|F_{2}(\ell, h)\right|^{2} d s\right)<\infty$. Since by assumption

$$
\mathbb{E} \exp \left(\frac{1}{2} \int_{0}^{t} \frac{1}{\sigma_{2}^{2}}\left|F_{2}(\ell(s), h(s))\right|^{2} d s\right) \leq \exp \left(\frac{K^{2}}{2 \sigma_{2}^{2}} t\right)<\infty,
$$

we know that the measures induced on path space by $\ell_{[0, t]}$ conditioned on $\eta$ and $z_{[0, t]}$ are equivalent. This in turn implies that the time $t$ marginals are equivalent. Since the law of $z(t)$ for fixed $t$ is Gaussian and thus equivalent to Lebesgue measure the proof is complete.

Proof of The SECOND PART OF LEMMA 8.2: We now prove the statements concerning $Q_{[0, \infty)}$ and $Q_{[0, \infty)}^{\eta}$. Again we use Girsanov's Theorem and only consider the conditioned measures. This time we compare the measures induced on $[0, t]$ by $\ell$ starting from the same $\ell_{0}$ with the same $\eta$ but different $h$ 's. In this case, Novikov's criterion becomes

$$
\begin{gathered}
\mathbb{E} \exp \left(\frac{1}{2} \int_{0}^{t} \frac{1}{\sigma_{2}^{2}}\left|F_{2}\left(\ell, \Phi_{0, s}^{\eta}\left(\ell_{[0, s]} ; h_{0}\right)\right)-F_{2}\left(\ell, \Phi_{0, s}^{\eta}\left(\ell_{[0, s]} ; \tilde{h}_{0}\right)\right)\right|^{2} d s\right) \\
\leq \exp \left(\frac{1}{2} \int_{0}^{t} \frac{L_{2}}{\sigma_{2}^{2}}\left|\Phi_{0, s}^{\eta}\left(\ell_{[0, s]} ; h_{0}\right)-\Phi_{0, s}^{\eta}\left(\ell_{[0, s]} ; \tilde{h}_{0}\right)\right|^{2} d s\right) \\
\leq \exp \left(\frac{1}{2} \int_{0}^{t} \frac{L_{2}}{\sigma_{2}^{2}}\left|h_{0}-\tilde{h}_{0}\right|^{2} e^{-2\left(\nu_{1}-L_{1}\right) s} d s\right) \\
\leq \exp \left(\frac{L_{2}}{4 \sigma_{2}^{2}\left(\nu_{1}-L_{1}\right)}\left|h_{0}-\tilde{h}_{0}\right|^{2}\right) .
\end{gathered}
$$

Since the bound is finite and uniformly bounded in $t$, we conclude that the measures on path space are equivalent on the time interval $[0, \infty)$.

\subsection{Basic Ergodicity}

We now present some general theorems which we will use to prove the ergodicity of the SNS equations and the toy model. Hopefully, the assumptions will seem natural 
in light of the structure of the toy model.

Assumption 1. There exists a set $\mathcal{B} \subset C(0, \infty ; \mathbb{X})$, with $\mathbb{P}\left\{\varphi_{[0, \infty)}^{W} u_{0} \in \mathcal{B}\right\}=1$ for all $u_{0} \in \mathbb{X}$ so that the following holds:

If $u(t, W)$ and $\tilde{u}(t, W)$ are solutions to (10) and $\Omega_{0}$ a subset of $\Omega \times \Omega$ so that

$$
(W, \tilde{W}) \in \Omega_{0} \Longrightarrow \begin{cases}u(\cdot, W), \tilde{u}(\cdot, \tilde{W}) \in \mathcal{B} & \\ \Pi_{h} W(t)-\Pi_{h} W(0)=\Pi_{h} \tilde{W}(t)-\Pi_{h} \tilde{W}(0) & \text { for all } t \geq 0 \\ \Pi_{\ell} u(t, W)=\Pi_{\ell} \tilde{u}(t, \tilde{W}) & \text { for all } t \geq 0\end{cases}
$$

then $\left|\Pi_{h} u(t, W)-\Pi_{h} \tilde{u}(t, \tilde{W})\right|_{\mathbb{X}} \rightarrow 0$ as $t \rightarrow \infty$ for all $(W, \tilde{W}) \in \Omega_{0}$.

In the toy model the set $\mathcal{B}$ was not needed; the conclusion held for all paths. This is not true in more general settings; we need to restrict ourselves to a set of "nice" paths. For the SNS equation, $\mathcal{B}$ will be the set of paths which grow and average in a typical fashion. Recall from (14), that $Q_{t}^{\eta}\left(u_{0}, \cdot\right)$ and $Q_{[t, \infty)}^{\eta}\left(u_{0}, \cdot\right)$ are respectively the measure induced on the "low modes" $\mathbb{X}_{\ell}$ at time $t$ by $u(t)$ and on the path space $C\left([0, \infty), \mathbb{X}_{\ell}\right)$ by $u_{[t, \infty)}$ if one conditions to use the noise realization $\eta$ and to start from the initial condition $u_{0}$ at time $t=0$.

Assumption 2. For all $u_{0}=\left(\ell_{0}, h_{0}\right) \in \mathbb{X}_{\ell} \times \mathbb{X}_{h}, Q_{t}^{\eta}\left(\ell_{0}, h_{0}, \cdot\right)$ is equivalent to Lebesgue measure for almost every $\eta$. For all $u_{0}=\left(\ell_{0}, h_{0}\right)$ and $\tilde{u}_{0}=\left(\tilde{\ell}_{0}, \tilde{h}_{0}\right) \in$ $\mathbb{X}_{\ell} \times \mathbb{X}_{h}$, the measure $Q_{[0, \infty)}^{\eta}\left(\ell_{0}, h_{0}, \cdot\right)$ is equivalent to $Q_{[0, \infty)}^{\eta}\left(\ell_{0}, \tilde{h}_{0}, \cdot\right)$ for almost every $\eta$.

As noted in the analysis of the toy problem, the equivalence of the measure conditioned on $\eta$ implies the equivalence of the unconditioned versions.

Theorem 6. If Assumptions 1 and 2 hold, then (10) has at most one $\mathbb{X}$-valued invariant probability measure.

By an $\mathbb{X}$-valued probability measure $\mu$, we mean a measure such that $\mu(\mathbb{X})=1$. Once this theorem is proven, we will have proven the ergodicity of the toy problem from the previous section. In the SNS setting, notice that Assumption 2 is close to Theorem 4. Lemma 13.1 makes the set $\mathcal{B}$ explicit. We now state a number of lemma which will be used to prove Theorem 6 .

Lemma 8.3. Assume Assumption 2 holds. For any pair of initial conditions $u_{0}=$ $\left(\ell_{0}, h_{0}\right)$ and $\tilde{u}_{0}=\left(\tilde{\ell}_{0}, \tilde{h}_{0}\right) \in \mathbb{X}_{\ell} \times \mathbb{X}_{h}$ and any $t>0$, the measure $Q_{[t, \infty)}\left(\ell_{0}, h_{0}, \cdot\right)$ and $Q_{[t, \infty)}\left(\tilde{\ell}_{0}, \tilde{h}_{0}, \cdot\right)$ are equivalent. Similarly for almost every $\eta, Q_{[t, \infty)}^{\eta}\left(\ell_{0}, h_{0}, \cdot\right)$ is equivalent to $Q_{[t, \infty)}^{\eta}\left(\tilde{\ell}_{0}, \tilde{h}_{0}, \cdot\right)$.

Given any invariant measure $\mu$, we define two classes of associated measures; one on the future trajectories and one on the past trajectories. Let $\mathcal{M}_{-}$denote the natural measure on $C((-\infty, 0] ; \mathbb{X})$ defined by cylinder sets of the type: for some $t_{0}, t_{1}, \cdots t_{n}, t_{0}<t_{1}<t_{2} \cdots t_{n} \leq 0$

$$
A=\left\{(\ell(s), h(s)) \in C((-\infty, 0], \mathbb{X}),\left(\ell\left(t_{i}\right), h\left(t_{i}\right)\right) \in A_{i}, i=0, \cdots n\right\}
$$


where the $A_{i}$ 's are Borel sets of $\mathbb{X}$. The definition

$$
\mathcal{M}_{-}(A)=\mu\left(A_{0}\right) \cdot \mathbb{P}\left\{u\left(t_{i}\right) \in A_{i}, i=1 \ldots n \mid u\left(t_{0}\right) \in A_{0}\right\} .
$$

characterizes the measure. Similarly we define $\mathcal{M}_{+}$on $C([0, \infty) ; \mathbb{X})$. We also define $\mathcal{M}_{+}^{\eta}$ on $C([0, \infty) ; \mathbb{X})$ by pushing $\mu$ forward under dynamics conditioned to use the noise realization $\eta$. We will define a measure $\mathcal{M}_{-}^{\eta}$ at the end of the section and explore it properties. Recalling that $\Pi_{\ell}$ was the projection onto $\mathbb{X}_{\ell}$; and hence, $\mathcal{M}_{+} \Pi_{\ell}^{-1}$ is a measure on $C\left([0, \infty) ; \mathbb{X}_{\ell}\right)$. Analogously for $t>s$, we define $\Pi_{[s, t)}$ as the projection onto the space $C([s, t) ; \mathbb{X})$.

Assumption 3. Let $\mu_{1}$ and $\mu_{2}$ be any two invariant measures and let $\mathcal{M}_{+, 1}^{\eta}$ and $\mathcal{M}_{+, 2}^{\eta}$ be the measures induced on $C([0, \infty) ; \mathbb{X})$ described above. Then for almost every $\eta, \mathcal{M}_{+, 1}^{\eta} \Pi_{\ell}^{-1}$ is equivalent to $\mathcal{M}_{+, 2}^{\eta} \Pi_{\ell}^{-1}$.

Lemma 8.4. Assumption 2 implies Assumption 3.

Proof of Lemma 8.4: Since $\mu$ is invariant, for any $B \subset C\left([t, \infty) ; \mathbb{X}_{\ell}\right)$ and $t>0$

$$
\mathcal{M}_{+}^{\eta} \Pi_{\ell}^{-1}(B)=\int_{\mathbb{X}} Q_{[t, \infty)}^{\theta-t \eta}\left(\ell_{0}, h_{0}, B\right) \mu\left(d \ell_{0} \times d h_{0}\right)
$$

the result follows from Lemma 8.3 and since $\theta_{t}$ is ergodic; hence, mapping one set of full measure in $\Pi_{\ell} \Omega$ to another set of full measure.

Assumption 3, is weaker than Assumption 2. As the next lemma shows, it is sufficient to prove ergodicity. In some settings where solutions to the initial value problem do not have nice moment properties it is more convenient to work directly with stationary solutions. This type of analysis is presented in [BM03]. However, in systems like the SNS equations such difficulties do not arise and, as we shall see, Assumption 2 holds.

In light of above lemma, the following result implies Theorem 6 .

Lemma 8.5. If Assumptions 1 and 3 hold, then (10) has at most one $\mathbb{X}$-valued invariant probability measure.

Note: If one was only interested in events which depended on the part of the path in $\mathbb{X}_{\ell}$ then Assumption 1 is not needed.

PROOF OF LEMMA 8.5: Since all invariant measures are a linear combination of ergodic measures it is enough to show there is a unique ergodic measure. Let $\mu_{1}$ and $\mu_{2}$ be two different ergodic measures. Let $\mathcal{M}_{+, 1}, \mathcal{M}_{+, 1}^{\eta}, \mathcal{M}_{+, 2}$ and $\mathcal{M}_{+, 2}^{\eta}$ be the associated measures defined above. Let $\phi: \mathbb{X}_{\ell} \times \mathbb{X}_{h} \rightarrow \mathbb{R}$ be a measurable test function bounded with $\sup |\phi|_{\mathbb{X}} \leq 1$ and $\phi(\ell, \cdot) \in \operatorname{Lip}_{1}\left(\mathbb{X}_{h}\right)$ for all $\ell$. The norm induced on measures by this class of test functions dominates the Wasserstein ( or Kantorovich) distance for measures. Hence, this class of test functions is rich enough so that if $\int \phi d \mu_{1}=\int \phi d \mu_{2}$ for all such $\phi$ then $\mu_{1}=\mu_{2}$ (cf. [Dud76] ). Since $\mathcal{M}_{+, i}$ is invariant under the flow induced on measures, the Birkoff ergodic theorem 
implies that there exists sets $\bar{A}_{i} \subset C([0, \infty), \mathbb{X})$ such that $\mathcal{M}_{+, i}\left(\bar{A}_{i}\right)=1$ and for all $(\ell, h) \in \bar{A}_{i}$

$$
\lim _{t \rightarrow \infty} \frac{1}{t} \int_{0}^{t} \phi(\ell(s), h(s)) d s=\bar{\phi}_{i}=\int \phi(x, y) \mu_{i}(d x \times d y) .
$$

Define $A_{i}=\bar{A}_{i} \cap \mathcal{B}$ where $\mathcal{B}$ is the set from Assumption 1. Again remark that $\mathcal{M}_{+, i}\left(A_{i}\right)=1$ since $\mathcal{B}$ has full measure. Since $\mathcal{M}_{+, i}\left(\bar{A}_{i}\right)=\mathbb{E} \mathcal{M}_{+, i}^{\eta}\left(\bar{A}_{i}\right)=1$, $\mathcal{M}_{+, i}^{\eta}\left(A_{i}\right)=1$ for almost every $\eta$. Let $A_{i}^{\eta}$ be a subset of $A_{i}$ of full $\mathcal{M}_{+, i}^{\eta}$-measure so that the paths in $A_{i}^{\eta}$ are solutions with a noise realization $W$ so $\Pi_{\ell} W=\eta$. By Assumption 3, $\mathcal{M}_{+, 1}^{\eta} \Pi_{\ell}^{-1}$ is equivalent to $\mathcal{M}_{+, 2}^{\eta} \Pi_{\ell}^{-1}$. Hence, $\mathcal{M}_{+, 2}^{\eta} \Pi_{\ell}^{-1}\left(\Pi_{\ell} A_{1}^{\eta}\right)>0$ which implies that $\Pi_{\ell} A_{1}^{\eta} \cap \Pi_{\ell} A_{2}^{\eta}$ is not empty since $\mathcal{M}_{+, 2}^{\eta} \Pi_{\ell}^{-1}\left(\Pi_{\ell} A_{2}^{\eta}\right)=1$. Hence, the set $A=\left\{\left(\ell, h_{1}, h_{2}\right):\left(\ell, h_{1}\right) \in A_{1}^{\eta}\right.$ and $\left.\left(\ell, h_{2}\right) \in A_{2}^{\eta}\right\}$ is not empty. Fixing some $\left(\ell, h_{1}, h_{2}\right) \in A$, from (19) and Assumption 1 we have that for any $\epsilon>0$ there exists a $T$ so that for all $t>T$

$$
\left|\frac{1}{t} \int_{0}^{t} \phi\left(\ell(s), h_{i}(s)\right) d s-\bar{\phi}_{i}\right|<\frac{\epsilon}{4}
$$

and $\left|h_{1}(t)-h_{2}(t)\right|_{\mathbb{X}}<\frac{\epsilon}{4}$. This last inequality holds because the hypotheses of Assumptions 1 are satisfied. Hence,

$$
\begin{aligned}
\left|\bar{\phi}_{1}-\bar{\phi}_{2}\right| & \leq \sum_{i}\left|\frac{1}{t} \int_{0}^{t} \phi\left(\ell(s), h_{i}(s)\right) d s-\bar{\phi}_{i}\right|+\left|\frac{1}{t} \int_{0}^{t} \phi\left(\ell(s), h_{1}(s)\right)-\phi\left(\ell(s), h_{2}(s)\right) d s\right| \\
& \leq \frac{\epsilon}{2}+\frac{2 T}{t}+\frac{\epsilon}{4} \frac{t-T}{t}<\epsilon \text { for } t \text { sufficiently large. }
\end{aligned}
$$

Since $\epsilon$ was arbitrary, the proof is complete.

Proof of Lemma 8.3: Let $A \subset C(0, \infty ; \mathbb{X})$. We will show that

$$
Q_{[t, \infty)}^{\eta}\left(\ell_{0}, h_{0}, A\right)=0 \text { implies that } Q_{[t, \infty)}^{\eta}\left(\tilde{\ell}_{0}, \tilde{h}_{0}, A\right)=0 .
$$

First notice that for $B \subset \Pi_{h} \mathbb{X}$ if $H_{t}^{\eta}\left(\ell_{0}, h_{0}, x, B\right)=\mathbb{P}\left\{h(t) \in B \mid \ell(0)=\ell_{0}, h(0)=\right.$ $\left.h_{0}, \ell(t)=x, \mathcal{F}_{[0, t]}^{\eta}\right\}$ for $t>0$ then

$$
Q_{[t, \infty)}^{\eta}\left(\ell_{0}, h_{0}, A\right)=\iint Q_{t}^{\eta}\left(\ell_{0}, h_{0}, d x\right) H_{t}^{\eta}\left(\ell_{0}, h_{0}, x, d y\right) Q_{[0, \infty)}^{\theta_{t} \eta}(x, y, A) .
$$

Hence for almost every $\eta, Q_{[t, \infty)}^{\eta}\left(\ell_{0}, h_{0}, A\right)=0$ implies that $Q_{[0, \infty)}^{\theta_{t} \eta}(x, y, A)=0$ for $\operatorname{Leb}(d x) \times H_{t}^{\eta}\left(\ell_{0}, h_{0}, x, d y\right)$ almost every $(x, y)$ because by assumption $Q_{t}^{\eta}\left(\ell_{0}, h_{0}, \cdot\right)$ is equivalent to Lebesgue measure. By the second part of Assumption 2, we know that $Q_{[0, \infty)}^{\tilde{\eta}}(x, y, \cdot)$ is equivalent to $Q_{[0, \infty)}^{\tilde{\eta}}(x, \tilde{y}, \cdot)$ for all $x, y, \tilde{y}$ and for $\mathbb{P}$-almost every $\tilde{\eta}$. Hence, $Q_{[0, \infty)}^{\theta_{t} \eta}(x, y, A)=0$ for $\operatorname{Leb}(d x) \times H_{t}^{\eta}\left(\tilde{\ell}_{0}, \tilde{h}_{0}, x, d y\right)$ almost every $(x, y)$ and $\mathbb{P}$-almost every $\eta$. (Here we have used that the shift is ergodic with respect to $\mathbb{P}$. So $\theta_{t}$ a maps set of full measure to another set of full measure.) And hence, by the representation for $Q_{[t, \infty)}^{\eta}\left(\tilde{\ell}_{0}, \tilde{h}_{0}, A\right)$ analogous to $(20)$, we conclude $Q_{[t, \infty)}^{\eta}\left(\tilde{\ell}_{0}, \tilde{h}_{0}, A\right)=0$. 


\subsection{One Force, One Solution: Statistical Equilibrium Measures and Trivial Random Attractors}

In analogy to $\mathcal{M}_{+}^{\eta}$, we define $\mathcal{M}_{-}^{\eta}$ on $C((-\infty, 0] ; \mathbb{X})$ as the limit as $t \rightarrow-\infty$ of $M_{t, 0}^{\eta}=\mathbb{E}\left\{\varphi_{[t, 0]}^{(\xi, \eta)} \mu \mid \mathcal{F}_{(-\infty, 0]}^{\eta}\right\}$. As discussed in [LJ87, DLJ88, Bax91], the sequence is a backwards martingale, hence the limit exists almost surely by the martingale convergence theorem. By $\varphi_{[t, 0]}^{(\xi, \eta)} u_{0}$ we mean the entire piece of trajectory on $[t, 0]$. Similarly, one can define $\mathcal{M}_{-}^{(\xi, \eta)}$ on $C((-\infty, 0] ; \mathbb{X})$ by the limit $t \rightarrow-\infty$ of $\varphi_{[t, 0]}^{(\xi, \eta)} \mu$. This is the called the equilibrium measure [LJ87] and $\mathcal{M}_{-}^{\eta}=\mathbb{E}\left\{\mathcal{M}_{-}^{(\xi, \eta)} \mid \mathcal{F}_{(-\infty, 0]}^{\eta}\right\}$. In a similar manner, one can define $\mathcal{M}^{\eta}$ on all of $C\left(-\infty, \infty ; \mathbb{X}_{\ell}\right)$.

In the case of the SNS equations Flandolli, Craul, and Debussche [Fla94, CDF97] proven the existence of a compact random attractor $\mathcal{A}(\xi, \eta) \subset \mathbb{L}^{2}$ which attracts all bounded subsets $B \subset \mathbb{L}^{2}$ in the sense

$$
\lim _{t \rightarrow-\infty} d\left(\varphi_{t, 0}^{(\xi, \eta)} B, \mathcal{A}(\xi, \eta)\right)=0
$$

where $d$ is the symmetric Hausdorff distance on sets.

If we define the action of the shift $\theta_{t}$ of $\eta$ as $\left(\theta_{t} \eta\right)(s)=\eta(t+s)-\eta(t)$, the measure $\mathcal{M}^{\eta}$ is invariant under the skew flow on measures fibered over $\eta$. Then we have, for example, $\mathbb{E}\left\{\varphi_{0, t}^{(\xi, \eta)} \mathcal{M}^{\eta} \mid \mathcal{F}^{\eta}\right\}=\mathcal{M}^{\theta_{t} \eta}$. (Recall that $\mathcal{F}^{\eta}$ was the sigma algebra generated by $\eta$.) Similarly, when a random attractor exists $\varphi_{0, t}^{(\xi, \eta)} \mathcal{A}(\xi, \eta)=\mathcal{A}\left(\theta_{t} \xi, \theta_{t} \eta\right)$.

We can consider the equation (11) in isolation over a probability space $\Pi_{h} \Omega \times$ $C\left(-\infty, \infty ; \mathbb{X}_{\ell}\right)$ with the measure $\mathbb{P}_{\eta}(d \eta) \times \mathcal{M}^{\eta} \Pi_{\ell}^{-1}(d \ell)$. In other words, we have elevated the part of the phase space $C\left(-\infty, \infty ; \mathbb{X}_{\ell}\right)$ to part of the base probability space. On this space the $h(t)$ dynamics has the same property as the whole SNS equation under the extremely contractive assumption $\left(N_{*}^{2} \geq \mathcal{C} \frac{\mathcal{E}_{0}}{\nu^{3}}\right)$. In particular, an analogous theorem to Theorem 5 holds: there is a unique solution $h_{*}(t ; \eta, \ell)$ which attracts all other solutions. In these coordinates, the random attractor for the equation (11) is the single solution $h_{*}(t ; \eta, \ell)$. Therefore, the invariant measure $\mu$ from above projected onto $\mathbb{X}_{h}$ disintegrates into a delta measure concentrated at $\delta_{h_{*}(0 ; \eta, \ell)}$ against the measure $\mathbb{P}_{\eta}(d \eta) \times \mathcal{M}^{\eta} \Pi_{\ell}^{-1}(d \ell)$. That is to say, if $F: \mathbb{X}_{\ell} \times \mathbb{X}_{h} \rightarrow \mathbb{R}$ then

$$
\begin{aligned}
\int F(\ell, h) \mu(d \ell \times d h) & =\int F\left(\ell, h_{*}(0 ; \eta, \ell)\right)\left[\mathbb{P}_{\eta}(d \eta) \times \mathcal{M}^{\eta} \Pi_{\ell}^{-1}(d \ell)\right] \\
& =\int F(\ell, h) \delta_{h_{*}(0 ; \eta, \ell)}(d h)\left[\mathbb{P}_{\eta}(d \eta) \times \mathcal{M}^{\eta} \Pi_{\ell}^{-1}(d \ell)\right] .
\end{aligned}
$$

This is the analog for the partially dissipative system of the "one force, one solution" (i.e. trivial random attractor) discussed in [Mat99, EKMS00, Sch97, LJ87, Mat02a, MY02, EVE00] or exemplified by Theorem 5. A similar statement holds for the toy problem and all of the systems satisfying the assumptions in section 8.2.

\section{Contractive Nature of the SNS Dynamics}

The proof of ergodicity of the SNS under the assumption that only the "determining modes" are forced will parallel the proof of the toy model. Our first step is to estab- 
lish Assumption 1 in the context of the SNS. We do this by proving a quantitative version of Theorem 4 which was given earlier.

To see what is involved we consider two solutions to equation (8), $h_{1}$ and $h_{2}$, driven by a common low mode process $\ell$ and noise $\eta$. That is for $t>s, h_{i}(t)=$ $\Phi_{s, t}^{\eta}\left(\ell_{[s, t)} ; h_{i}(s)\right)$. Denoting $u_{i}=\ell+h_{i}$, we have

$$
\frac{\partial \rho(t)}{\partial t}=-\Lambda^{2} \rho(t)+\Pi_{h} B\left(u_{1}, \rho\right)+\Pi_{h} B\left(\rho, u_{2}\right)
$$

which using standard estimates on the nonlinearity (cf. [CF88]) produces, for some $\mathcal{C}>0$

$$
\begin{aligned}
\frac{d|\rho|_{\mathbb{L}^{2}}^{2}}{d t} & \leq-2 \nu|\Lambda \rho|_{\mathbb{L}^{2}}^{2}+(2 \mathcal{C})^{\frac{1}{2}}|\Lambda \rho|_{\mathbb{L}^{2}}\left|\Lambda u_{2}\right|_{\mathbb{L}^{2}}|\rho|_{\mathbb{L}^{2}} \leq-\nu|\Lambda \rho|_{\mathbb{L}^{2}}^{2}+\frac{\mathcal{C}}{\nu}\left|\Lambda u_{2}\right|_{\mathbb{L}^{2}}^{2}|\rho|_{\mathbb{L}^{2}}^{2} \\
& \leq-\left(\nu N_{*}^{2}-\frac{\mathcal{C}}{\nu}\left|\Lambda u_{2}\right|_{\mathbb{L}^{2}}^{2}\right)|\rho|_{\mathbb{L}^{2}}^{2} .
\end{aligned}
$$

The above estimate then gives for $t_{0}<t$

$$
|\rho(t)|_{\mathbb{L}^{2}}^{2} \leq\left|\rho\left(t_{0}\right)\right|_{\mathbb{L}^{2}}^{2} \exp \left(-\nu N_{*}^{2}\left(t-t_{0}\right)+\frac{\mathcal{C}}{\nu} \int_{t_{0}}^{t}\left|\Lambda u_{2}(s)\right|_{\mathbb{L}^{2}}^{2} d s\right)
$$

We now see the new difficulty which the Stochastic Navier Stokes equations present over the toy model. The contraction rate depends on the time average of the enstropy $\left|\Lambda u_{2}(s)\right|_{\mathbb{L}^{2}}^{2}$ of one of the solutions. However, after we develop some estimates controlling this quantity the proof will proceed using standard ideas of localization from stochastic analysis.

\section{The Energy and Enstrophy}

The toy model is an extremely uniform setting. The added difficulty in the SNS relative to the toy model, is the lack of uniformity. However, the standard idea of localization from stochastic analysis allows us to overcome this hurdle. As we saw in the last section, the growth of the energy $|u|_{\mathbb{L}^{2}}^{2}$ and the time average of the enstrophy $|\Lambda u|_{\mathbb{L}^{2}}^{2}$ seem to be of importance in controlling the uniformity of the contraction. This will be come clearer after the next two sections. We begin with some estimates on the energy and enstrophy.

Lemma 10.1. $\mathbb{E}|u(t)|_{\mathbb{L}^{2}}^{2} \leq e^{-2 \nu\left(t-t_{0}\right)} \mathbb{E}\left|u\left(t_{0}\right)\right|_{\mathbb{L}^{2}}^{2}+\frac{\mathcal{E}_{0}}{2 \nu}\left(1-e^{-2 \nu\left(t-t_{0}\right)}\right)$ and for any $p \geq 1, \mathbb{E}|u(t)|_{\mathbb{L}^{2}}^{2 p} \leq e^{-2 \nu\left(t-t_{0}\right)} \mathbb{E}\left|u\left(t_{0}\right)\right|_{\mathbb{L}^{2}}^{2 p}+C_{0} \int_{t_{0}}^{t} e^{-2 \nu(t-s)} \mathbb{E}|u(s)|_{\mathbb{L}^{2}}^{2(p-1)} d s$.

This implies that if one has a solution $u(t, W)$ defined for $t \in(-\infty, \infty)$ such that $e^{-2 \nu|t|} \mathbb{E}|u(t, W)|_{\mathbb{L}^{2}}^{2} \rightarrow 0$ as $t \rightarrow-\infty$ then in fact $\mathbb{E}|u(t, W)|_{\mathbb{L}^{2}}^{2}$ is uniformly bounded in time. Using similar reasoning, one can show the following result.

Lemma 10.2. Assume that $\mu$ is an invariant measure such that there exist a $U \subset \mathbb{L}^{2}$ with $\mu(U)=1$. For any such measure stationary measure all energy moments are finite. In fact for any $p \geq 1$ there exist constant $C_{p}<\infty$ such 
that $\int_{\mathbb{L}^{2}}|u|_{\mathbb{L}^{2}}^{2 p} d \mu(u)<C_{p}$ for all invariant measures $\mu$. In particular, $C_{1}=\frac{\mathcal{E}_{0}}{2 \nu}$. Furthermore, $\int_{\mathbb{L}^{2}}|\Lambda u|_{\mathbb{L}^{2}}^{2} d \mu(u)=\frac{\mathcal{E}_{0}}{2 \nu}$ assuming only that $\mathcal{E}_{0}=\sum\left|\sigma_{k}\right|^{2}<\infty$. If $\mathcal{E}_{1}=\sum|k|^{2}\left|\sigma_{k}\right|^{2}<\infty$ is finite then the analogous statements hold for $|\Lambda u|_{\mathbb{L}^{2}}^{2 p}$ replacing $|u|_{\mathbb{L}^{2}}^{2 p}$. In particular, $\int_{\mathbb{L}^{2}}\left|\Lambda^{2} u\right|_{\mathbb{L}^{2}}^{2} d \mu(u)=\frac{\mathcal{E}_{1}}{2 \nu}$.

Since one can construct a stationary solution from any invariant measure and vice versa (see section 8.2 ), this conclusion applies equally to any stationary solution. The proofs of Lemma 10.1 and 10.2 can be found in the appendix of [EMS01]. Related statements can be found in Chapter 3 and 4 of [Mat98], section 2 of [Mat99], or the appendix of [Mat02b]. The moment estimates are just the stochastic analogs of deterministic estimates. Similar estimates from slightly different points of view can be found in [VF88, MR, BKL00]. If one assumes, $\mathcal{E}_{1}=\sum\left|\sigma_{k}\right|^{2}|k|^{2}<\infty$ then completely analogous statements can be made about the enstrophy.

It is critical to our analysis to understand the typical size of the fluctuations of the enstrophy about its mean of $\frac{\mathcal{E}_{0}}{2 \nu}$. Applying Itô's formula to the energy, one obtains

$$
d|u(s)|_{\mathbb{L}^{2}}^{2}=-2 \nu|\Lambda u(s)|_{\mathbb{L}^{2}}^{2} d t+\mathcal{E}_{0} d t+2\langle u, d W(t)\rangle_{\mathbb{L}^{2}}
$$

If one writes the last term as

$$
\left(\sum_{k}\left|\sigma_{k} u_{k}\right|^{2}\right)^{\frac{1}{2}}\left[\frac{\langle u, d W(t)\rangle_{\mathbb{L}^{2}}}{\left(\sum_{k}\left|\sigma_{k} u_{k}\right|^{2}\right)^{\frac{1}{2}}}\right],
$$

then the term in the square brackets is distributed as a one dimensional Brownian motion adapted to the filtration generated by the $W$ increments. This motivates our definition of a Lyapunov function in the abstract setting (10), which is contained in the next section.

\section{Growth and Fluctuations in A General Setting}

In this section, we put an abstract framework on the ideas of the previous section. In the section 12 , we return to the concrete setting of the SNS.

Assumption 4. There exists a function $V: \mathbb{X} \rightarrow[0, \infty)$, with $c_{0} V(x)^{p_{0}} \geq|x|_{\mathbb{X}}^{2}$ for positive $p_{0}, c_{0}$, so that for a solution $u$ of equation (10) $V$ satisfies the Itô equation

$$
d V(u(t))=g(u(t)) d t+f(u(t)) d \widetilde{B}(t) .
$$

Here $\widetilde{B}$ is a standard one dimensional Wiener process adapted to the flow generated by $(d W) . g: \mathbb{X} \rightarrow \mathbb{R}$ is a function satisfying

$$
g(u)<C_{1}-U(u) \text { where } U(u) \geq C_{2} V(u)
$$

for some constants $C_{1}, C_{2}>0$ and $U: \mathbb{X} \rightarrow[0, \infty) \cup \infty$. Though $U$ might be infinite on $\mathbb{X}$, we assume if $u(t)$ is a solution to $(10)$ on $[0, t]$, then $\int_{0}^{t} U(u(s)) d s<\infty$ almost surely. And $f: \mathbb{X} \rightarrow \mathbb{R}$ is a function satisfying

$$
C_{3}|f(u)|^{2} \leq U(u)
$$

for some $C_{3}>0$. 
From the calculation at the end of the last section, in the SNS setting we should take $V(u)=|u|_{\mathbb{L}^{2}}^{2}, U(u)=2 \nu|\Lambda u|_{\mathbb{L}^{2}}^{2}, C_{1}=\mathcal{E}_{0}, C_{2}=2 \nu$, and $C_{3}=\frac{\nu}{2 \sigma_{*}^{2}}$. (We could have also used $V(u)=|\Lambda u|_{\mathbb{L}^{2}}^{2}, U(u)=2 \nu\left|\Lambda^{2} u\right|_{\mathbb{L}^{2}}^{2}$ if $\mathcal{E}_{1}=\sum\left|\sigma_{k}\right||k|^{2}<\infty$.)

Lemma 11.1. For any $\epsilon \in(0,1)$ and $K>\frac{1}{\epsilon C_{3}}$ one has

$$
\mathbb{P}\left\{\sup _{t>0} \frac{V(u(t))+(1-\epsilon) \int_{0}^{t} U(u(s)) d s-V\left(u_{0}\right)-C_{1} t}{1+\log (1+t)}>K\right\} \leq \exp \left(-2 C_{3} \epsilon K\right)
$$

Proof: Let $M(t)$ denote the martingale $\int_{0}^{t} f(u(s)) d \widetilde{W}(s)$. Its quadratic variation $[M, M](t)$ is $\int_{0}^{t} f(u(s))^{2} d s$. Since $C_{3}[M, M](t) \leq \int_{0}^{t} U(u(s)) d s$, by Itô's formula we have

$$
V(u(t))+(1-\epsilon) \int_{0}^{t} U(u(s)) d s-V\left(u_{0}\right)-C_{1} t \leq M(t)-\epsilon C_{3}[M, M](t)
$$

The exponential martingale estimate implies that

$$
\mathbb{P}\left\{\sup _{t \leq T} M(t)-\epsilon C_{3}[M, M](t)>a\right\} \leq e^{-2 \epsilon C_{3} a} .
$$

Setting $a=K[1+\log (T)]$ one sees that probability of the event in the statement of the lemma is bounded from above by

$$
\sum_{n=1}^{\infty} \exp \left(-2 C_{3} \epsilon K[1+\log (n)]\right) \leq \int_{1}^{\infty} \exp \left(-2 \epsilon C_{3} K-2 \log (x)\right) d x=\exp \left(-2 C_{3} \epsilon K\right)
$$

More details can be found in the proofs of the following related results: Theorem 4-6 in [BM03], Lemma A.5 and Lemma B.3 of [EMS01], or for the use of the exponential martingale Lemma A.2 [Mat02c].

Using similar reasoning one can prove (see for example [BKL00, Mat02b]):

Lemma 11.2. There exist positive constants $\gamma$ and $K$ so that for all invariant measures $\mu$ with $V(u)<\infty \mu$-almost surely, $\int \exp (\gamma V(u)) d \mu(u) \leq K<\infty$ and for every initial condition $u_{0}$ and $t \geq 0, \mathbb{E} \exp (\gamma V(u(t))) \leq K \exp \left(\gamma V\left(u_{0}\right)\right)$

We now give estimates backward in time for stationary solutions.

Lemma 11.3. Let $u(t, W)$ be a stationary solution to $(10)$ with $V(u(t))<\infty$ almost surely. There exists a $K_{0}$ and $a \gamma>0$ so that for $K>K_{0}$

$$
\mathbb{P}\left\{\sup _{t \in \mathbb{R}} \frac{V(u(t))+(1-\epsilon)\left|\int_{0}^{t} U(u(t)) d s\right|-C_{1}|t|}{1+\log (1+|t|)}>K\right\} \leq \exp (-\gamma K)
$$


Proof: The proof is essentially the same as that of Lemma (11.1). We write

$$
\begin{aligned}
\sup _{t \in[T-1, T]} V(u(t))+(1-\epsilon)\left|\int_{0}^{t} U(u(t)) d s\right|-C_{1}|t| \leq V(u(T-1))+ \\
\sup _{t \in[T-1, T]}\left[V(u(t))+(1-\epsilon)\left|\int_{0}^{t} U(u(s)) d s\right|-C_{1}|t|-V(u(T-1))\right]
\end{aligned}
$$

By the previous two lemmas both of these terms have exponential moments uniform in $T$. Using the same reasoning as in the end of the proof of Lemma 11.1 completes to proof.

In light of Lemmas 11.1 and 11.3 we define the following sets of "nice" trajectories which average well and grow in a typical fashion. Fixing some fixed $\epsilon_{*} \in(0,1)$, which will be set differently in different contexts, we define

$$
\begin{aligned}
& A_{n}=\left\{u \in C(-\infty, 0 ; \mathbb{X}): \sup _{t \leq 0} \frac{V(u(t))+\left(1-\epsilon_{*}\right)\left|\int_{0}^{t} U(u(s)) d s\right|-C_{1} t}{1+\log (t+1)} \leq n\right\}, \\
& B_{n}=\{u \in C(0, \infty ; \mathbb{X}): \\
& \left.\sup _{t \geq 0} \frac{V(u(t))+\left(1-\epsilon_{*}\right)\left|\int_{0}^{t} U(u(s)) d s\right|-V\left(u_{0}\right)-C_{1} t}{1+\log (1+t)} \leq n\right\} .
\end{aligned}
$$

The previous lemmas imply that, with probability one, any stationary solution is contained in $\cup A_{n}$ and the solution to any initial value problem is contained in $\cup B_{n}$.

From these lemmas it is clear that

$$
\lim _{t \rightarrow \infty} \frac{1}{t} \int_{0}^{t} U(u(s)) d s \leq C_{1} \quad \text { and } \quad \lim _{t \rightarrow-\infty} \frac{1}{|t|} \int_{t}^{0} U(u(s)) d s \leq C_{1}
$$

almost surely. This is the result analogous to the final conclusion of Lemma 10.2. But notice that Lemma 11.1 and 11.3 also give information about the size of the fluctuations.

\section{Strongly Contractive Case SNS: Proof of Theorem 5}

In the next section, we will take a more abstract point of view on the contractive nature of the SNS equation and other SPDE. However, first for illustrative reasons, we continue with the explicit calculations began in section 9 and use them to prove Theorem 5 .

Proof of TheOREM 5: We begin by proving uniqueness. Let us assume that there are two solutions $u(t, W)$ and $u_{*}(t, W)$ defined for all $t \in(-\infty, \infty)$. Let $\rho(t, W)=u(t, W)-u_{*}(t, W)$. Both are governed by (3), so subtracting produces

$$
\frac{\partial \rho(t)}{\partial t}=-\Lambda^{2} \rho(t)+B(u, \rho)+B\left(\rho, u_{*}\right)
$$


Using the same estimates on the nonlinearity as in (21), we obtain

$$
\frac{d|\rho|_{\mathbb{L}^{2}}^{2}}{d t} \leq-\left(\nu N_{*}^{2}-\frac{\mathcal{C}}{\nu}\left|\Lambda u_{*}\right|_{\mathbb{L}^{2}}^{2}\right)|\rho|_{\mathbb{L}^{2}}^{2}
$$

Notice that this is the same estimate as was obtained in (21). Since $u_{*}$ is a solution, we know that it is contained in some $A_{n}$, where $A_{n}$ was the set of "nicely" fluctuating and growing paths defined in the last section. Recall that for the SNS: $V(u)=|u|_{\mathbb{L}^{2}}^{2}$, $U(u)=2 \nu|\Lambda u|_{\mathbb{L}^{2}}^{2}, C_{1}=\mathcal{E}_{0}$. Hence, $u \in A_{n}$ implies that for $t \leq 0$

$$
|u(t)|_{\mathbb{L}^{2}}^{2}+\left.\left(1-\epsilon_{*}\right) 2 \nu\left|\int_{0}^{t}\right| \Lambda u(s)\right|_{\mathbb{L}^{2}} ^{2} d s\left|-\mathcal{E}_{0}\right| t \mid<n[1+\log (|t|+1)]
$$

where $\epsilon_{*} \in(1,0)$ is a free parameter which we will set momentarily. Continuing the estimation of $\rho$, using this bound, produces for $t_{0}<0$

$$
\begin{aligned}
|\rho(0)|_{\mathbb{L}^{2}}^{2} & \leq\left|\rho\left(t_{0}\right)\right|_{\mathbb{L}^{2}}^{2} \exp \left(-\nu N_{*}^{2}\left|t_{0}\right|+\frac{\mathcal{C}}{\nu} \int_{t_{0}}^{0}\left|\Lambda u_{2}(s)\right|_{\mathbb{L}^{2}}^{2} d s\right) \\
& \leq\left|\rho\left(t_{0}\right)\right|_{\mathbb{L}^{2}}^{2} \exp \left(-\left[\nu N_{*}^{2}-\mathcal{C} \frac{\mathcal{E}_{0}}{\nu^{2}\left(1-\epsilon_{*}\right)}\right]\left|t_{0}\right|+\frac{n\left[1+\log \left(\left|t_{0}\right|+1\right)\right]}{2 \nu\left(1-\epsilon_{*}\right)}\right) .
\end{aligned}
$$

Picking $\epsilon_{*}$ so $\nu N_{*}^{2}-\mathcal{C} \frac{\mathcal{E}_{0}}{\nu^{2}\left(1-\epsilon_{*}\right)}=\frac{1}{2} \gamma_{*}$ where $\gamma_{*}=\nu N_{*}^{2}-\mathcal{C} \frac{\mathcal{E}_{0}}{\nu^{2}}$ and using the assumption that the solutions are in $A_{n}$ to control $\left|\rho\left(t_{0}\right)\right|_{\mathbb{L}^{2}}^{2}$ yields the estimate

$$
|\rho(0)|_{\mathbb{L}^{2}}^{2} \leq 4\left[\mathcal{E}_{0}+n\left(1+\log \left(1+\left|t_{0}\right|\right)\right] \exp \left(-\frac{1}{2} \gamma_{*}\left|t_{0}\right|+\frac{n\left[1+\log \left(\left|t_{0}\right|+1\right)\right]}{2 \nu\left(1-\epsilon_{*}\right)}\right) .\right.
$$

Taking $t_{0} \rightarrow-\infty$, proves uniqueness. A similar estimate shows that the solution to any initial value problem converges exponentially forward in time to $u_{0}$. The existence can be deduced from the existence of a stationary measure; however, it is instructive to construct it directly, which we now do.

Let $u_{n}(t)$ be the solution starting from initial value zero at time $-n$. From Lemma 11.1, we know that $\theta_{-n} u_{n} \in B_{k_{n}}$ for some $k_{n}$. $\quad\left(\theta_{-n}\right.$ just shifts the path on $[-n, \infty)$ to a path on $[0, \infty)$.) In addition, we know that $\mathbb{P}\left\{k_{n}>n^{\frac{1}{8}}\right\} \leq$ $\exp \left(-\frac{\nu \epsilon_{*}}{\sigma_{*}^{2}} n^{\frac{1}{8}}\right)$. Hence, by the Borel-Cantelli Lemma, there exists an $n_{*}$ so that $k_{n} \leq n^{\frac{1}{8}}$ for all $n>n_{*}$. Let $n>m>M>n_{*}$, then for $M$ sufficiently large we have

$$
\begin{aligned}
& \sup _{s \in[-1,0]}\left|u_{n}(s)-u_{m}(s)\right|_{\mathbb{L}^{2}} \leq \sum_{j=m+1}^{n} \sup _{s \in[-1,0]}\left|u_{j+1}(s)-u_{j}(s)\right|_{\mathbb{L}^{2}} \\
& \leq \sum_{j=M}^{\infty} \sup _{s \in[-1,0]}\left|u_{j+1}(s)-u_{j}(s)\right|_{\mathbb{L}^{2}} \\
& \leq \sum_{j=M}^{\infty}\left|u_{j+1}(-j)\right|_{\mathbb{L}^{2}} \exp \left(-\frac{1}{2} \gamma_{*}|j-1|+|j|^{\frac{1}{8}}[1+\log (1+|j|)]\right) \\
& \leq \sum_{j=M}^{\infty}\left[\mathcal{E}_{0}+2|j|^{\frac{1}{8}}\right] \exp \left(-\frac{1}{2} \gamma_{*}|j-1|+|j|^{\frac{1}{8}}[1+\log (1+|j|)]\right)
\end{aligned}
$$


Since the last sum is less than $C \exp (-\gamma M)$ for some positive constants $C$ and $\gamma$, the sequence is Cauchy and the proof is complete.

\section{Contractive Nature in the General Setting}

We now extract the essential assumptions of the previous section and present them in an abstract form. The choice of assumptions follows [BM03] which uses ever so slightly different assumptions, but proves more detailed estimates. In particular, statements about the continuity of the map $\Phi$ are made. (See Theorem 9 of [BM03].) The treatment is also informed and influenced by [Hai02, Mat02c, EL02].

Assumption 5. Consider the $G: \mathbb{X} \rightarrow \mathbb{X}$ from (10). Assume that Assumption 4 holds and that for all $\ell \in \mathbb{X}_{\ell}$ and $h, \tilde{h} \in \mathbb{X}_{h}$ and some $c_{i} \geq 0$, and $p_{i} \geq 0$, with $c_{1}>C_{1}\left(c_{2}+c_{3}\right)$,

$$
\begin{aligned}
&\langle G(\ell+h)-G(\ell+\tilde{h}), h-\tilde{h}\rangle_{\mathbb{X}} \leq\left[-c_{1}+c_{2} U(\ell+h)+c_{3} U(\ell+\tilde{h})\right]|h-\tilde{h}|_{\mathbb{X}}^{2} \\
&\left|\Pi_{\ell} G(\ell+h)-\Pi_{\ell} G(\ell+\tilde{h})\right|_{\mathbb{X}}^{2} \leq c_{4}\left[1+V(\ell+h)^{p_{1}}+V(\ell+\tilde{h})^{p_{1}}\right]|h-\tilde{h}|_{\mathbb{X}}^{p_{2}}
\end{aligned}
$$

We give the analog of Theorem 4 and Lemma 8.1 in the general setting of equation (10). This is a quantitative version of the determining mode result given in Theorem 4 and will be used to verify Assumption 1 .

Lemma 13.1. Let Assumption 5 hold. In particular, $\gamma_{*} \stackrel{\text { def }}{=} c_{1}-C_{1}\left(c_{2}+c_{3}\right)>0$. Set the $\epsilon_{*}$ from the definition of $A_{n}$ and $B_{n}$ in (23), so that $c_{1}-\frac{C_{1}\left(c_{2}+c_{3}\right)}{1-\epsilon_{*}}=\frac{1}{2} \gamma_{*}$ and define $c_{*}=\frac{c_{2}+c_{3}}{1-\epsilon_{*}}$.

1. Fixing a $T \in(0, \infty]$, let $\ell \in C\left(0, T ; \mathbb{X}_{\ell}\right)$ and $h_{0}, \tilde{h}_{0} \in \mathbb{X}_{h}$ and define $u(t)=$ $\ell(t)+\Phi_{0, t}^{\eta}\left(\ell_{[0, t]} ; h_{0}\right)$ and $\tilde{u}(t)=\ell(t)+\Phi_{0, t}^{\eta}\left(\ell_{[0, t]} ; \tilde{h}_{0}\right)$.

Assume that $u \in \Pi_{[0, T)} B_{n}$ if $c_{2}>0$ and $\tilde{u} \in \Pi_{[0, T)} B_{n}$ if $c_{3}>0$. Then for all $t \in[0, T)$

$$
\left|\Phi_{0, t}^{\eta}\left(\ell_{[0, t]} ; h_{0}\right)-\Phi_{0, t}^{\eta}\left(\ell_{[0, t]} ; \tilde{h}_{0}\right)\right|_{\mathbb{X}}^{2} \leq\left|h_{0}-\tilde{h}_{0}\right|_{\mathbb{X}}^{2} e^{n c_{*}}(1+t)^{n c_{*}} e^{-\frac{1}{2} \gamma_{*} t} .
$$

2. Fixing a $T \in(-\infty, 0)$, let $\ell \in C\left(T, 0 ; \mathbb{X}_{\ell}\right)$ and $h_{0}, \tilde{h}_{0} \in \mathbb{X}_{h}$ and define $u(t)=$ $\ell(t)+\Phi_{T, t}^{\eta}\left(\ell_{[T, t]} ; h_{0}\right)$ and $\tilde{u}(t)=\ell(t)+\Phi_{T, t}^{\eta}\left(\ell_{[T, t]} ; \tilde{h}_{0}\right)$.

Assume that $u \in \Pi_{[T, 0]} A_{n}$ if $c_{2}>0$ and $\tilde{u} \in \Pi_{[T, 0]} A_{n}$ if $c_{3}>0$. Then for all $t \in[T, 0]$

$$
\left|\Phi_{T, t}^{\eta}\left(\ell_{[T, t]} ; h_{0}\right)-\Phi_{T, t}^{\eta}\left(\ell_{[T, t]} ; \tilde{h}_{0}\right)\right|_{\mathbb{X}}^{2} \leq\left|h_{0}-\tilde{h}_{0}\right|_{\mathbb{X}}^{2} e^{n c_{*}}(1+|T|)^{n c_{*}} e^{-\frac{1}{2} \gamma_{*}(|T|-|t|)} .
$$

This theorem can be restated in terms of solutions to (10).

Corollary 13.2. Let $\Omega_{0} \subset \Omega \times \Omega$ and $c_{*}$ and $\gamma_{*}$ are as defined in Lemma 13.1. 
1. Let $u(t, W)$ and $\tilde{u}(t, W)$ be solutions to (10) on $C(0, T ; \mathbb{X})$ with $T>0$. If for $(W, \tilde{W}) \in \Omega_{0}$, one has $u(W), \tilde{u}(\tilde{W}) \in \Pi_{[0, T]} B_{n}$ and $\Pi_{h} W(s)-\Pi_{h} W(T)=$ $\Pi_{h} \tilde{W}(s)-\Pi_{h} W(T), \Pi_{\ell} u(s, W)=\Pi_{\ell} \tilde{u}(s, \tilde{W})$ for $s \in[0, T]$ then

$$
|u(t, W)-\tilde{u}(t, \tilde{W})|_{\mathbb{X}}^{2} \leq|u(0, W)-\tilde{u}(0, \tilde{W})|_{\mathbb{X}}^{2} e^{n c_{*}}(1+t)^{n c_{*}} e^{-\frac{1}{2} \gamma_{*} t} .
$$

2. Let $u(t, W)$ and $\tilde{u}(t, W)$ be solutions to (10) on $C(T, 0 ; \mathbb{X})$ with $T<0$. If for $(W, \tilde{W}) \in \Omega_{0}$, one has $u(W), \tilde{u}(\tilde{W}) \in \Pi_{[T, 0]} A_{n}$ and $\Pi_{h} W(s)-W(T)=$ $\Pi_{h} \tilde{W}(s)-W(T), \Pi_{\ell} u(s, W)=\Pi_{\ell} \tilde{u}(s, \tilde{W})$ for $s \in[T, 0]$ then

$$
\begin{aligned}
|u(t, W)-\tilde{u}(t, \tilde{W})|_{\mathbb{X}}^{2} \leq 2\left[C_{1}|T|+n+n \log (1+|T|)\right] e^{n c_{*}} \times & \\
& (1+|T|)^{n c_{*}} e^{-\frac{1}{2} \gamma_{*}(|T|-|t|)} .
\end{aligned}
$$

Proof of Lemma 13.1 And Corollary 13.2: The proof of the two statements is almost identical and is simply an abstraction of the ideas in the proof of Theorem 5 given in the last section. We give the details of the first statement.

Let $\rho(s)=\Phi_{0, s}^{\eta}\left(\ell_{[0, s]} ; h_{0}\right)-\Phi_{0, s}^{\eta}\left(\ell_{[0, s]} ; \tilde{h}_{0}\right)$, then equation (11) and the assumption in the lemma and Assumption 5 imply that

$$
\begin{aligned}
\frac{1}{2} \frac{d|\rho(s)|_{\mathbb{X}}^{2}}{d t} & =\langle G(u)-G(\tilde{u}), \rho(s)\rangle_{\mathbb{X}} \\
& \leq\left[-c_{1}+c_{2} U(u(s))+c_{3} U(\tilde{u}(s))\right]|\rho(s)|_{\mathbb{X}}^{2} .
\end{aligned}
$$

Since $u, \tilde{u} \in \Pi_{[0, T]} B_{n}$, we have

$$
|\rho(t)|_{\mathbb{X}}^{2} \leq|\rho(0)|_{\mathbb{X}}^{2} \exp \left(-\left[c_{1}-\frac{C_{1}\left(c_{2}+c_{3}\right)}{1-\epsilon_{*}}\right] t+n \frac{c_{2}+c_{3}}{1-\epsilon_{*}}(1+\log (1+t))\right)
$$

which proves the first result. The second result is just the same except that the estimates from $A_{n}$ are used. See the proof of Theorem 5. Corollary 13.2 is just a restatement of the theorem with the added observation that backwards in time the initial conditions $h_{0}=\Pi_{h} u(T, W)$ and $\tilde{h}_{0}=\Pi_{h} \tilde{u}(T, \tilde{W})$ can not grow too fast since the solutions are in $A_{n}$.

Using the contractive properties backward in time one can define the limit

$$
\lim _{t \rightarrow-\infty} \Phi_{t, 0}^{\eta}\left(\ell ; h_{0}\right)
$$

for any $\ell$ which is a projection of a solution $u(t, W)$ on $(-\infty, 0]$. This limiting function, denoted $\Phi(\ell)$, is independent of $h_{0}$ and can be used to reduce the dynamics to one on $\mathbb{X}_{\ell}$ with memory (i.e. Gibbsian dynamics). See [BM03] discussion of this in a general setting and [EMS01, EL02] for specific examples. If one endows $C(-\infty, 0 ; \mathbb{X})$ with the metric $|u|_{r}=\sup _{t<0} \frac{|u(t)|_{\mathbb{X}}}{1+|t|^{r}}$, then in many settings $\Phi(\ell)$ is continuous on the set of solutions $u$ with $\ell=\Pi_{\ell} u$. In fact under some simple assumptions, it is globally Lipschitz on each $B_{n}$ defined in section 11 . In particular, both of these facts hold for the SNS equation. See [BM03] for more discussion of this. 


\section{Ergodicity: the SNS and the General Setting}

We now turn to completing the proof of Theorem 3. All that remains to prove is the last part of Theorem 3 about the "essentially elliptic" dynamics (the case when $\left.N_{*}^{2}>\mathcal{C} \frac{\mathcal{E}_{0}}{\nu^{3}}\right)$. We will do so by proving an ergodic theorem in the general setting of (10) and using the assumptions already introduced.

To prove basic ergodicity in this case, we will use Theorem 6, which along with Corollary 13.2, contains the essential ideas from [EMS01]. Lemma 11.1 implies that almost every solution $\varphi_{[0, \infty)}^{W} u_{0}$ is contained in a $B_{n}$ for some $n$. This, coupled with Lemma 13.2, is more than enough to imply Assumption 1 of Section 8.2 with $\mathcal{B}=\cup B_{n}$. We need only verify Assumption 2, to prove complete the proof. Since the author feels that techniques often used to verify the first part of Assumption 2 are suboptimal, we leave it as an assumption for the moment. We will revisit the question at the end of this section. Hence, we introduce the following assumption.

Assumption 6. For all $t>0$ and $\left(\ell_{0}, h_{0}\right) \in \mathbb{X}$ and almost every $\eta, Q_{t}^{\eta}\left(\ell_{0}, h_{0}, \cdot\right)$ is equivalent to Lebesgue measure.

The idea to prove the second part of Assumption 2 is again the idea of localization. By restricting ourselves to well behaved paths, we will be able to of obtain the needed result for a subset of the probability space. By relaxing the restriction, we can include arbitrarily large subsets of the probability space, implying that the conclusion holds with probability one. We prove the following result.

Theorem 7. Consider equation (10). Let Assumption 4, 5 and 6 hold. In addition if $\sigma_{k}>0$ for all $k$ with $|k| \in\left(0, N_{*}\right)$, where $N_{*}$ was used to define the splitting of equation (10), then the system has at most one invariant measure.

Note: It is worth mentioning that existence of an invariant measure in our setting is usually straight forward. For instance, if the set $\{u: V(u) \leq M\}$ is precompact for all $M$ then the result follows easily by the standard Krylov-Bogoljubov construction of extracting a convergent subsequence from the empirical measures obtained by time-averaging. See for instance [CK97] for the SPDE setting or [CFS82] for general discussions.

We begin the proof of Theorem 7 by proving the analog of Lemma 8.2 from the discussion of the toy model. In fact, we will only deduce part of it from our existing assumptions leaving the remainder still as an assumption.

Lemma 14.1. Consider the solution to equation (10) under the assumptions of Theorem 14.1. For $\ell_{0} \in \mathbb{X}_{\ell}$ and $h_{0}, \tilde{h}_{0} \in \mathbb{X}_{h}$ and almost every $\eta, Q_{[0, \infty)}^{\eta}\left(\ell_{0}, h_{0}, \cdot\right)$ is equivalent to $Q_{[0, \infty)}^{\eta}\left(\ell_{0}, \tilde{h}_{0}, \cdot\right)$.

Proof of Lemma 14.1: Again we begin by essentially localizing to a fixed $B_{n}$. However, we need to pick a set of paths in $C\left(0, \infty ; \mathbb{X}_{\ell}\right)$. Fixing $\ell_{0}, h_{0}, \tilde{h}_{0}$ and $\eta$, we define

$$
\begin{aligned}
B_{n}^{\prime}=\left\{\ell_{[0, \infty)}: u, \tilde{u} \in B_{n} \text { where } u(s)=\ell(s)+\Phi_{s}^{\eta}\left(\ell_{[0, s]}, h_{0}\right),\right. & \\
& \left.\tilde{u}(s)=\ell(s)+\Phi_{s}^{\eta}\left(\ell_{[0, s]}, \tilde{h}_{0}\right)\right\}
\end{aligned}
$$


Then for $A \subset C\left(0, \infty ; \mathbb{X}_{\ell}\right)$, define

$$
Q_{[0, t]}^{\eta}\left(\ell_{0}, h_{0}, A ; B_{n}^{\prime}\right)=\mathbb{P}\left\{u_{[0, t]} \in A ; \Pi_{\ell} u_{[0, t]} \in B_{n}^{\prime} \mid u(0)=\left(\ell_{0}, h_{0}\right)\right\}
$$

We now compare $Q_{[0, t]}^{\eta}\left(\ell_{0}, h_{0}, \cdot ; B_{n}^{\prime}\right)$ to $Q_{[0, t]}^{\eta}\left(\ell_{0}, \tilde{h}_{0}, \cdot ; B_{n}^{\prime}\right)$. Again we compare the measure using Lemma A.1 from the appendix. By restricting to $B_{n}^{\prime}$, we ensure that both $u$ and $\tilde{u}$ stay in $B_{n}$. Hence, the first part of Lemma 13.1 combined with the second estimate in Assumption 5 produces

$$
\begin{aligned}
& \left|\Pi_{\ell} G(u(t))-\Pi_{\ell} G(\tilde{u}(t))\right|_{\mathbb{X}}^{2} \leq c_{4}\left[1+2\left(C_{1} t+n[1+\log (1+t)]\right)^{p_{1}}\right] \times \\
& \left|\Phi_{0, t}^{\eta}\left(\Pi_{\ell} u_{[0, t]} ; h_{0}\right)-\Phi_{0, t}^{\eta}\left(\Pi_{\ell} u_{[0, t]} ; \tilde{h}_{0}\right)\right|_{\mathbb{X}}^{p_{2}}
\end{aligned}
$$

where

$$
\left|\Phi_{0, t}^{\eta}\left(\Pi_{\ell} u_{[0, t]} ; h_{0}\right)-\Phi_{0, t}^{\eta}\left(\Pi_{\ell} u_{[0, t]} ; \tilde{h}_{0}\right)\right|_{\mathbb{X}}^{2} \leq\left|h_{0}-\tilde{h}_{0}\right|_{\mathbb{X}}^{2} e^{n c_{*}}(1+t)^{n c_{*}} e^{-\frac{1}{2} \gamma_{*} t} .
$$

Defining $\sigma_{\text {min }}^{2}=\min _{|k|<N_{*}}\left|\sigma_{k}\right|^{2}$, the previous two estimates imply that

$$
\int_{0}^{\infty} \frac{1}{\sigma_{\min }^{2}}\left|\Pi_{\ell} G(u(t))-\Pi_{\ell} G(\tilde{u}(t))\right|_{\mathbb{X}}^{2} d t<D_{*}<\infty
$$

for some $D_{*}$ uniformly on $B_{n}^{\prime}$. Using Lemma A.1, we conclude that $Q_{t}^{\eta}\left(\ell_{0}, h_{0}, \cdot ; B_{n}^{\prime}\right)$ is equivalent to $Q_{t}^{\eta}\left(\ell_{0}, \tilde{h}_{0}, \cdot ; B_{n}^{\prime}\right)$. As in the previous part, since both $u$ and $\tilde{u}$ are in $\cup B_{n}$ with probability one, we conclude that $Q_{t}^{\eta}\left(\ell_{0}, h_{0}, \cdot\right)$ is equivalent to $Q_{t}^{\eta}\left(\ell_{0}, \tilde{h}_{0}, \cdot\right)$.

Looking back on the above proof, we seen that there was a great deal of uniformity in the estimates. When comparing $Q_{[0, t]}^{\eta}\left(\ell_{0}, h_{0}, \cdot ; B_{n}^{\prime}\right)$ to $Q_{[0, t]}^{\eta}\left(\ell_{0}, \tilde{h}_{0}, \cdot ; B_{n}^{\prime}\right)$, we see that for all $\left(\ell_{0}, h_{0}\right),\left(\ell, \tilde{h}_{0}\right)$ in a bounded ball, we can choose the same $D_{*}$. From Lemma A.1 in the appendix, we get the following result

Lemma 14.2. For any $M$, there exists a $D_{*}$ so that if $\left|\ell_{0}+h_{0}\right|_{\mathbb{X}},\left|\ell_{0}+\tilde{h}_{0}\right|_{\mathbb{X}} \leq M$ then

$$
\mathbb{E}\left[\frac{d Q_{[0, t]}^{\eta}\left(\ell_{0}, h_{0}, \cdot ; B_{n}^{\prime}\right)}{d Q_{[0, t]}^{\eta}\left(\ell_{0}, \tilde{h}_{0}, \cdot ; B_{n}^{\prime}\right)}\right]^{p} \leq D_{*}^{p(p-1)}
$$

for all $p>0$.

Conclusion of the Proof of Theorem 7: In light of Lemma 14.1 and 13.1 the result follows from Theorem 6 .

We now address Assumption 6. In the case of the stochastic Navier Stokes equations it is implied without further assumptions by the techniques used to prove Theorem 10 from section 17 since all of the directions in $\mathbb{X}_{\ell}$ are directly forced. (Theorem 10 does not address the question of the marginal with respect to $\eta$. However Theorem 10 follows from the fact that the Malliavin covariance matrix restricted 
to $\mathbb{X}_{\ell}$ is almost surely invertible. This does imply the result for the marginals. See [MP03].)

The same techniques should apply to most SPDEs of interest with additive noise. However, since an abstract version of the techniques in [MP03] is not written, we refrain from making any claims. There is however another approach. Though it is rather adhoc and in the author's opinion and "not the correct way," it is sufficient in many contexts. The basic idea is to compare the measures induced on $C\left(0, t ; \mathbb{X}_{\ell}\right)$ by the process of interest and some well understood process both starting from the same point. This is done using Girsonov's theorem. Then the time $t$ marginals of the well understood process starting from two different points are compared. By stringing the estimates together and making some additional assumptions needed to control the "high" modes in equation (8), one can prove Assumption 6. A particularly simple version of this was done in the toy model of the previous section. For more complicated versions see [EMS01, EL02, Mat02c, BM03]. [BM03] has a relatively crisp version of the argument.

\section{Exponential Mixing and Coupling}

In this section, we expand the simple uniqueness results, given earlier in the paper, by giving a rate of convergence. The proof will be based on a coupling argument and is closer in packaging to the author's first proof of basic ergodicity which were presented in seminar talks ${ }^{1}$. We will measure the rate of convergence of (10) using the following metric. For any two measures $\mu_{1}$ and $\mu_{2}$ on $\mathbb{X}$ define

$$
\left\|\mu_{1}-\mu_{2}\right\|_{*}=\sup _{\phi \in \mathcal{G}_{*}} \int \phi(x) \mu_{1}(d x)-\int \phi(x) \mu_{2}(d x)
$$

where $\mathcal{G}_{*}$ is the set of all measureable functions $\phi: \mathbb{X} \rightarrow \mathbb{R}$ with $|\phi(u)| \leq 1$ for all $u \in \mathbb{X}$ and $|\phi(\ell+h)-\phi(\ell+\tilde{h})| \leq|h-\tilde{h}|_{\mathbb{X}}$ for all $\ell \in \mathbb{X}_{\ell}$ and $h, \tilde{h} \in \mathbb{X}_{h}$. Notice that the $\|\cdot\|_{*}$ norm dominates the Wasserstein or Kantorovich distance for measures but is weaker than the total variation norm. In the definition of $\mathcal{G}_{*}$, we could have also used test function which were $\operatorname{Lip}_{\alpha}$ on $\mathbb{X}_{h}$, with $\alpha>0$, and all of the theorems below would still hold.

Now we make the following assumption which is a more qualitative version of Assumption 6. It amounts to continuity in the initial condition of the density induced on $\mathbb{X}_{\ell}$ at time $t$.

Assumption 7. Fix any $t>0$. For any $M_{0}$, there exist positive $a \delta$ and $\Omega^{\prime} \subset \Pi_{h} \Omega$ so $\mathbb{P}\left(\eta \in \Omega^{\prime}\right)>\delta$ and for any $\eta \in \Omega^{\prime}$ and $u_{0}^{(i)} \in \mathbb{X}, i=1,2$, with $V\left(u_{0}^{(i)}\right) \leq M_{0}$ we have $\frac{1}{2}\left\|Q_{t}^{\eta}\left(u_{0}^{(1)}, \cdot\right)-Q_{t}^{\eta}\left(u_{0}^{(2)}, \cdot\right)\right\|_{T V}<1-\delta$.

See Appendix B for the definition of $\|\cdot\|_{T V}$ which may differ by a factor of 2 from some definitions. Again this estimate can be obtained in a number of ways. For the SNS it was obtained by comparing, in a quantitative fashion, the total variation distance between the time $t$ marginals and well controlled reference process (either

\footnotetext{
${ }^{1}$ Stanford and Berkeley probability seminars November and December 1999.
} 
Brownian motion or the SDE on $\mathbb{X}_{\ell}$ obtained from the Galerkin truncation of the SNS). However the author feels that this is not the optimal fashion to proceed. It would be better to use the flow property and the calculations from [MP03] to verify this estimate. Since the assumption has only been verified in specific cases, we leave it as an assumption.

Letting $P_{t}\left(u_{0}, A\right)=\mathbb{P}\left\{u(t) \in A \mid u(0)=u_{0}\right\}$ where $A \subset \mathbb{X}$, we have the following result whose proof give in the sections which follow. Stronger results using norms allowing test functions which grow are also possible by the methods presented here. Corollary 15.1 at the end of the section gives a simple, suboptimal example. See [MT93] or [MSH02] for examples to the type of stronger statements which should be possible. However [MT93, MSH02] does not apply to our setting.

Theorem 8. If Assumption 4, 5 and 7 hold, then there exists fixed positive constants $K$ and $\gamma$ so for all $u_{0}^{(i)} \in \mathbb{X}$ (possibly random, but adapted to the filtration at time zero) (see notes below.)

$$
\left\|P_{t}\left(u_{0}^{(1)}, \cdot\right)-P_{t}\left(u_{0}^{(1)}, \cdot\right)\right\|_{*} \leq K\left[1+\mathbb{E} V\left(u_{0}^{(1)}\right)+\mathbb{E} V\left(u_{0}^{(2)}\right)\right] e^{-\gamma t}
$$

We give the proof of this theorem in the next sections. In [Mat02c] a general theorem, ensuring exponential mixing in a wide class of problems, was given and the conditions were verified for the SNS. However, given the estimates of the previous section the exact same analysis applies to equation (10) when $\eta=\Pi_{h} W=0$. In that paper, the case $\eta=\Pi_{h} W \neq 0$ was discussed in another setting. A straight forward modification of the techniques from that paper yields the extension to $\eta=\Pi_{h} W \neq 0$. Kuksin and Shirikyan were the first to consider exponential mixing for the SNS in the case when $\eta \neq 0$ [KS02]; however, their norm is slightly weaker. The norm we give here gives total variation convergence on a subset of the space which dictates the asymptotic behavior, namely $\mathbb{X}_{\ell}$. This allows on to use standard mixing results to get law of large numbers, central limit theorems, and other results. With additional work this also possible directly in the framework of [KS02] or [Hai02]. See [Shi02]

In [Mat02c], the case $\eta \neq 0$ was considered in a simple map example and we see here that those ideas extend to the SPDE context. In [BKL02], exponential convergence was proven but without the explicit dependence on the initial condition. That paper along with [Mat02c] were the first proofs of exponential convergence of the SNS with white in time forcing. In the kicked case exponential convergence was given in [MY02, KPS02]. The first of these also considers the the case where the system is strongly dissipative as in Theorem 5. In [Hai02], exponential convergence for a reaction diffusion equation was proved by bringing the paths together asymptotically using a coupling construction inspired by [Mat02c]. (Both [Mat02c] and [BKL02] were delayed considerable in the review process, and hence, [Hai02] appeared first.)

To state a slightly stronger result, for any weighing function $R: \mathbb{X} \rightarrow[0, \infty)$ define $\left\|\mu_{1}-\mu_{2}\right\|_{R *}=\sup _{\phi \in \mathcal{G}_{R^{*}}} \int \phi(x) \mu_{1}(d x)-\int \phi(x) \mu_{2}(d x)$ where $\mathcal{G}_{R *}$ is the set of all measureable functions $\phi: \mathbb{X} \rightarrow \mathbb{R}$ with $|\phi(u)| \leq R(u)$ for all $u \in \mathbb{X}$ and $|\phi(\ell+h)-\phi(\ell+\tilde{h})| \leq[1+R(\ell+h)+R(\ell+\tilde{h})]|h-\tilde{h}|_{\mathbb{X}}$ for all $\ell \in \mathbb{X}_{\ell}$ and $h, \tilde{h} \in \mathbb{X}_{h}$. 
Corollary 15.1. In the same setting as Theorem 8 , for any $\frac{1}{q}+\frac{1}{p}=1$ with $q, p>1$

$$
\begin{aligned}
\left\|P_{t}\left(u_{0}^{(1)}, \cdot\right)-P_{t}\left(u_{0}^{(1)}, \cdot\right)\right\|_{R *} \leq\left[1+\left(\mathbb{E} R(u(t))^{q}\right)^{\frac{1}{q}}+\left(\mathbb{E} R(\tilde{u}(t))^{q}\right)^{\frac{1}{q}}\right] \times \\
K^{\prime}\left[1+\mathbb{E} V\left(u_{0}^{(1)}\right)+\mathbb{E} V\left(u_{0}^{(2)}\right)\right]^{\frac{1}{p}} e^{-\gamma^{\prime} t}
\end{aligned}
$$

where $\gamma^{\prime}$ and $K^{\prime}$ are positive constants depending on $p$ and $q$.

Notice that if $R(x)=V(x)$ then the assumptions of the corollary are satisfied and $\mathbb{E} V(u(t))^{q} \leq K^{\prime \prime}\left[1+\mathbb{E} V\left(u_{0}\right)^{q}\right]$ for some $K^{\prime \prime}$ as $V^{q}$ is also a Lyapunov function. This Lemma is suboptimal as the right hand side does not scale linearly in $V\left(u_{0}\right)$ so a convenient operator norm is not induced. See [MSH02] for ideas, from the Markov setting, which likely could overcome this difficnency.

\subsection{Deconstruction and Reconstruction}

We begin with an overview of the coupling construction. The idea is to factor the measure induced on $C\left(0, \infty ; \mathbb{X}_{\ell}\right)$ starting from $u_{0}$ and $\tilde{u}_{0}$ and build a process on $C(0, \infty ; \mathbb{X}) \times C(0, \infty ; \mathbb{X})$ so that the marginals are distributed as a process started from $u_{0}$ and $\tilde{u}_{0}$ respectively and so that $\Pi_{\ell} u(t)=\Pi_{\ell} \tilde{u}(t)$ with positive probability. There is the added complication that we need to also have the processes use the same realization of $\eta=\Pi_{h} W$ and that we need to localize the trajectories to the nicely growing and averaging paths so that $\Pi_{h} u(t)-\Pi_{h} \tilde{u}(t)$ will converge to zero at a controlled rate. We begin with the localization.

The $B_{n}$ defined in section 11 were sufficient for localizing to prove uniqueness. They also showed how typical paths stayed in a logarithmic envelope about the average behavior. However the probability from deviating from a given $B_{n}$ after time $t$ decays slowly. To prove exponential convergence, we now localize with sets from which it becomes exponentially unlikely to deviate over time. For positive $M$ define

$$
\begin{aligned}
B(M)=\left\{u \in C(0, \infty ; \mathbb{X}): V(u(t))+\left(1-\epsilon_{*}\right) \int_{0}^{t} U(u(s)) d s-V\left(u_{0}\right)\right. & \\
& \left.\leq M+C_{1}\left(1+\epsilon_{*}\right) t \text { for all } t \geq 0\right\}
\end{aligned}
$$

The constant $\epsilon_{*}$ is chosen so that $c_{1}-\frac{1+\epsilon_{*}}{1-\epsilon_{*}} C_{1}\left(c_{2}+c_{3}\right)=\frac{1}{2} \gamma_{*}$. Recall that $\gamma_{*}=$ $c_{1}-C_{1}\left(c_{2}+c_{3}\right)$ was assumed positive. Clearly $\mathbb{P}\left\{u \in \cup_{M=1}^{\infty} B(M)\right\}=1$ and (22), $\mathbb{P}\left\{u_{[0, t]} \in \Pi_{[0, t]} B(M) ; u \notin B(M)\right\}$ decays exponential in $t$. Furthermore given the choice $\epsilon_{*}$, Lemma 13.1 (part one) holds with $B_{n}$ replaced with $B(M)$ and different constants on the right hand side of the decay estimate. Precisely, if $u_{[0, T)}^{(i)} \in \Pi_{[0, T)} B(M)(i=1,2)$ where $u^{(i)}(t)=\ell(t)+\Phi_{0, t}^{\eta}\left(\ell_{[0, t]} ; h_{0}^{(i)}\right)$ then for $t \in(0, T]$

$$
\left|\Phi_{0, t}^{\eta}\left(\ell_{[0, t]} ; h_{0}^{(1)}\right)-\Phi_{0, t}^{\eta}\left(\ell_{[0, t]} ; h_{0}^{(2)}\right)\right|_{\mathbb{X}}^{2} \leq\left|h_{0}^{(1)}-h_{0}^{(2)}\right|_{\mathbb{X}}^{2} e^{M} e^{-\frac{1}{2} \gamma_{*} t}
$$


Fix some $M$. For every $\left(u_{0}^{(1)}, u_{0}^{(2)}, \eta\right) \in \mathbb{X} \times \mathbb{X} \times \Pi_{h} \Omega$, we define

$$
\begin{array}{r}
\bar{B}_{[0, n]}\left(u_{0}^{(1)}, u_{0}^{(2)}, \eta\right)=\left\{\ell_{[0, n]} \in \Pi_{\ell} \mathbb{X}_{[0, n]}: u_{[1, n]}^{(i)} \in \Pi_{[0, n-1)} B(M),\left|u^{(i)}(1)\right|_{\mathbb{X}}^{2} \leq M\right. \\
\text { where } \left.u^{(i)}(s)=\ell(s)+\Phi_{s}^{\eta}\left(\ell_{[0, s]}, \Pi_{h} u_{0}^{(i)}\right)\right\}
\end{array}
$$

and define for $A \subset \prod_{\ell} \mathbb{X}_{[0, n-1)}$ the measure

$$
\begin{aligned}
Q_{[1, n]}^{\eta}\left(u_{0}, A ; \bar{B}_{[0, n]}\right) & =\mathbb{P}\left\{\Pi_{\ell} u_{[1, n]} \in A \text { and } \Pi_{\ell} u_{[0, n]} \in \bar{B}_{[0, n]} \mid u(0)=u_{0}, \mathcal{F}_{[0, n]}^{\eta}\right\} \\
& =\mathbb{E}\left\{\mathbf{1}_{A}\left(\Pi_{\ell} u_{[1, n]}\right) \mathbf{1}_{\bar{B}_{[0, n]}}\left(\Pi_{\ell} u_{[0, n]}\right) \mid u(0)=u_{0}, \mathcal{F}_{[0, n]}^{\eta}\right\}
\end{aligned}
$$

where $\bar{B}_{[0, n]}=\bar{B}_{[0, n]}\left(u_{0}^{(1)}, u_{0}^{(2)}, \eta\right)$. Hence $Q_{[1, n]}^{\eta}\left(u_{0}, A ; \bar{B}_{[0, n]}\right)$ is the measure of paths so that $\Pi_{\ell} u_{[0, n]} \in \bar{B}_{[0, n]}$ and $\Pi_{\ell} u_{[1, n]} \in A$ if one conditions to start from $u_{0}$ and use noise realizations $W$ so $\Pi_{\ell} W=\eta$. Of course, it is not a probability measure as it does not have total mass one.

Given any two measures $\mu_{1}$ and $\mu_{2}$, one can always write them as a density against a common third measure. That is $\mu_{i}(d x)=f_{i}(x) \mu_{3}(d x)$ for $i=1,2$. We define the measures $\mu_{1} \wedge \mu_{2}$ and $\mu_{1}-\mu_{2}$ respectively by the densities $\left(f_{1}(x) \wedge\right.$ $\left.f_{2}(x)\right) \mu_{3}(d x)$ and $\left(f_{1}(x)-f_{2}(x)\right) \mu_{3}(d x)$. It is easy to see that this definition is independent of the choice of $\mu_{3}$. If $\mu_{1}$ does not dominate $\mu_{2}$ for all measurable sets then the second measure is a signed measure. See Appendix B more explination and the realtion to the total variation norm, which we denote by $\|\cdot\|_{T V}$.

With this notation define

$$
\Psi_{n}^{\eta}\left(u_{0}^{(1)}, u_{0}^{(2)}, \cdot\right)=Q_{[1, n)}^{\eta}\left(u_{0}^{(1)}, \cdot ; \bar{B}_{[0, n)}\right) \wedge Q_{[1, n)}^{\eta}\left(u_{0}^{(2)}, \cdot ; \bar{B}_{[0, n)}\right)
$$

where again $\bar{B}_{[0, n)}=\bar{B}_{[0, n)}\left(u_{0}^{(1)}, u_{0}^{(2)}, \eta\right)$. Next for $A \subseteq \mathbb{X}_{[0, n)}$ and $\ell \in \Pi_{\ell} \mathbb{X}_{[0, n-1)}$ define

$$
H_{n}^{\eta}\left(u_{0}, A \mid \ell\right)=\mathbb{P}\left\{u_{[0, n)} \in A \mid u(0)=u_{0}, \Pi_{\ell} u_{[1, n)}=\ell_{[0, n-1)}, \mathcal{F}_{[0, n)}^{\eta}\right\} .
$$

In words $H_{n}^{\eta}\left(u_{0}, \cdot \mid \ell\right)$ is the measure induced on $\mathbb{X}_{[0, n)}$ by paths $u_{[0, n)}$ conditioned to start at $u_{0}$, use noise realization $\eta$, and such that $u(s+1)=\ell(s)$ for $s \in[0, n-1)$.

Next we define the two families of measures $\mathbf{r}_{n}$ and $\mathbf{s}_{n}, n \in\{1,2, \ldots, \infty\}$, which will be critical in our construction. They will both be measures on $\Pi_{\ell} \mathbb{X}_{[0, n)} \times$ $\Pi_{\ell} \mathbb{X}_{[0, n)} \times \Pi_{h} \Omega_{[0, n)}$ with $n \in\{1,2, \ldots, \infty\}$. In general, we will use bold letters to denote measures on such spaces and capital bold letters for probability measures on such spaces. Define

$$
\begin{aligned}
\mathbf{s}_{n}\left(u_{0}, \tilde{u}_{0}, d u \times d \tilde{u}\right. & \times d \eta) \\
= & \int_{\Pi_{\ell} \mathbb{X}_{[0, n-1)}}\left[H_{n}^{\eta}\left(u_{0}, d u \mid \ell\right) \times H_{n}^{\eta}\left(\tilde{u}_{0}, d \tilde{u} \mid \ell\right)\right] \Psi_{n}^{\eta}\left(u_{0}, \tilde{u}_{0}, d \ell\right) \times \mathbb{P}(d \eta)
\end{aligned}
$$

and

$$
\mathbf{r}_{n+1}\left(u_{0}, \tilde{u}_{0}, d u \times d \tilde{u} \times d \eta\right)=\left[\mathbf{P}_{1} \mathbf{s}_{n}-\mathbf{s}_{n+1}\right]\left(u_{0}, \tilde{u}_{0}, d u \times d \tilde{u} \times d \eta\right)
$$


Here $\mathbf{P}_{1} \mathbf{s}_{n}$ is the measure on $\Pi_{\ell} \mathbb{X}_{[0, n+1)} \times \Pi_{\ell} \mathbb{X}_{[0, n+1)} \times \Pi_{h} \Omega_{[0, n+1)}$ obtained by first stepping with $\mathbf{s}_{n}$ and then with

$\mathbf{P}_{n}\left(u_{0}, \tilde{u}_{0}, d u_{[0, n)} \times d \tilde{u}_{[0, n)} \times d \eta_{[0, n)}\right)=P_{[0, n)}^{\eta}\left(u_{0}, d u_{[0, n)}\right) \times P_{[0, n)}^{\eta}\left(\tilde{u}_{0} d \tilde{u}_{[0, n)}\right) \times \mathbb{P}\left(d \eta_{[0, n)}\right)$

where for $A \subset \mathbb{X}_{[0, n)}$ and $P_{[0, n)}^{\eta}\left(u_{0}, A\right)=\mathbb{P}\left\{u_{[0, n)} \in A \mid u(0)=u_{0}, \mathcal{F}_{[0, n)}^{\eta}\right\}$. That is to say,

$$
\begin{array}{r}
\mathbf{P}_{1} \mathbf{s}_{n}\left(u_{0}, \tilde{u}_{0}, d u_{[0, n+1)} \times d \tilde{u}_{[0, n+1)} \times d \eta_{[0, n+1)}\right)=\mathbf{s}_{n}\left(u_{0}, \tilde{u}_{0}, d u_{[0, n)} \times d \tilde{u}_{[0, n)} \times d \eta_{[0, n)}\right) \times \\
\mathbf{P}_{1}\left(u(n), \tilde{u}(n), d u_{[n, n+1)} \times d u_{[n, n+1)} \times d \eta_{[n, n+1)}\right) .
\end{array}
$$

Define $\mathbf{r}_{1}\left(u_{0}, \tilde{u}_{0}, \cdot\right)=\mathbf{P}_{1}\left(u_{0}, \tilde{u}_{0}, \cdot\right)-\mathbf{s}_{1}\left(u_{0}, \tilde{u}_{0}, \cdot\right)$. Since $\mathbf{P}_{1} \mathbf{s}_{n}\left(u_{0}, \tilde{u}_{0}, A\right) \geq$ $\mathbf{s}_{n+1}\left(u_{0}, \tilde{u}_{0}, A\right)$ by construction for all measurable sets $A, \mathbf{r}_{n}$ is a standard measure and not a signed measure. Lastly, we define

$$
\rho_{n}\left(u_{0}, \tilde{u}_{0}\right)=\mathbf{s}_{n}\left(u_{0}, \tilde{u}_{0}, \mathbb{X}_{[0, n)} \times \mathbb{X}_{[0, n)} \times \Pi_{h} \Omega_{[0, n)}\right)
$$

for $n>0$ (including $n=\infty$ ) and $\rho_{0}=1$ and the probability transition kernels

$$
\mathbf{S}_{n}\left(u_{0}, \tilde{u}_{0}, \cdot\right)=\frac{\mathbf{s}_{n}\left(u_{0}, \tilde{u}_{0}, \cdot\right)}{\rho_{n}\left(u_{0}, \tilde{u}_{0}\right)} \quad \mathbf{R}_{n}\left(u_{0}, \tilde{u}_{0}, \cdot\right)=\frac{\mathbf{r}_{n}\left(u_{0}, \tilde{u}_{0}, \cdot\right)}{\rho_{n-1}\left(u_{0}, \tilde{u}_{0}\right)-\rho_{n}\left(u_{0}, \tilde{u}_{0}\right)} .
$$

If the denominator is zero in either of the above definitions, we set the corresponding measure to the zero measure. Observe that

$$
\begin{aligned}
\rho_{n}\left(u_{0}, \tilde{u}_{0}\right) & =\mathbb{E} \Psi_{n}^{\eta}\left(u_{0}, \tilde{u}_{0}, \Pi_{\ell} \mathbb{X}_{[0, n-1)}\right) \\
& =1-\frac{1}{2} \mathbb{E}\left\|Q_{[1, n)}^{\eta}\left(u_{0}, \cdot ; \bar{B}_{[0, n)}\right)-Q_{[1, n)}^{\eta}\left(\tilde{u}_{0}, \cdot ; \bar{B}_{[0, n)}\right)\right\|_{T V}>0,
\end{aligned}
$$

where $\bar{B}_{[0, n)}=\bar{B}_{[0, n)}\left(u_{0}, \tilde{u}_{0}, \eta\right)$. This holds even for $n=\infty$, since for all $n$ the measures are absolutely continuous for almost every $\eta$. This can be seen by the same calculations as in the proof of Lemma 14.1 coupled will Lemma 8.3. Also observe that $\rho_{n}\left(u_{0}, \tilde{u}_{0}\right) \geq \rho_{n+1}\left(u_{0}, \tilde{u}_{0}\right)$. Thus, we have

$$
1=\rho_{0} \geq \rho_{1} \geq \cdots \geq \rho_{\infty}
$$

For all $M$ sufficiently large, we will see in Lemma 15.2 that $\rho_{\infty}\left(u_{0}, \tilde{u}_{0}\right)>0$ for all $u_{0}, \tilde{u}_{0}$ with $V\left(u_{0}\right), V\left(\tilde{u}_{0}\right) \leq M_{0}$.

From the properties of $\mathbf{s}_{n}$ and $\mathbf{r}_{n}$, one had $\mathbf{P}_{1}\left(u_{0}, \tilde{u}_{0}, \cdot\right)=\mathbf{s}_{1}\left(u_{0}, \tilde{u}_{0}, \cdot\right)+$ $\mathbf{r}_{1}\left(u_{0}, \tilde{u}_{0}, \cdot\right)$ and

$$
\begin{aligned}
\mathbf{P}_{2} & \stackrel{\mathrm{M}}{=} \mathbf{P}_{1} \mathbf{s}_{1}+\mathbf{P}_{1} \mathbf{r}_{1} \stackrel{\stackrel{M}{=}}{=} \mathbf{s}_{2}+\left[\mathbf{P}_{1} \mathbf{s}_{1}-\mathbf{s}_{2}\right]+\mathbf{r}_{1} \\
& \stackrel{\mathrm{M}}{=} \mathbf{s}_{2}+\mathbf{r}_{2}+\mathbf{r}_{1}
\end{aligned}
$$

where we have suppressed the dependence of the kernels on the initial conditions $u_{0}$ and $\tilde{u}_{0}$. By $\stackrel{\mathrm{M}}{=}$ we mean that the two measures have the same relevant marginals. More precisely if we consider the kernel at the point $\left(u_{0}, \tilde{u}_{0}\right)$, the joint distribution of the first and last coordinate of both sides is $P_{[0,2)}^{\eta}\left(u_{0}, \cdot\right) \times \mathbb{P}(d \eta)$ and the joint 
distribution of the second and last coordinate of both sides is $P_{[0,2)}^{\eta}\left(\tilde{u}_{0}, \cdot\right) \times \mathbb{P}(d \eta)$. Continuing along this line and normalizing the measures to probability measures, produces the following version of the factoring lemma from [Mat02c].

$$
\mathbf{P}_{\infty}\left(u_{0}, \tilde{u}_{0}, \cdot\right) \stackrel{\mathrm{M}}{=} \rho_{\infty} \mathbf{S}_{\infty}+\sum_{n=1}^{\infty}\left[\rho_{n-1}-\rho_{n}\right] \mathbf{P}_{\infty} \mathbf{R}_{n}
$$

where $\mathbf{P}_{\infty} \mathbf{R}_{n}$ is analogous to $\mathbf{P}_{1} \mathbf{s}_{n}$ from above. On the right hands side we have suppressed the dependence on $u_{0}$ and $\tilde{u}_{0}$ in the interest of space. That is $\mathbf{S}_{\infty}=$ $\mathbf{S}_{\infty}\left(u_{0}, \tilde{u}_{0}, \cdot\right), \rho_{n-1}=\rho_{n-1}\left(u_{0}, \tilde{u}_{0}\right)$ and so forth.

Such a factorization of the futures was also fundamental to the results in [Hai02]. Since the project of this measure onto the first and last coordinate of both sides equals $P_{[0, \infty)}^{\eta}\left(u_{0}, \cdot\right) \times \mathbb{P}\left(d \eta_{[0, \infty)}\right)$ and the projection on the second and last coordinate of both sides is $P_{[0, \infty)}^{\eta}\left(\tilde{u}_{0}, \cdot\right) \times \mathbb{P}\left(d \eta_{[0, \infty)}\right)$, we have built a representation of two copies of the process which both use the same $\eta$. The first is distributed as a solution starting from $u_{0}$ and the second as a solution starting from $\tilde{u}_{0}$. This representation has the the following importantly feature. There exists a set $A \subset \mathbb{X}_{[0, \infty)} \times \mathbb{X}_{[0, \infty)} \times$ $\Pi_{h} \Omega_{[0, \infty)}$ so that $\mathbf{S}_{\infty}(A)=1$ and if $(u, \tilde{u}, \eta) \in A$ then $\Pi_{\ell} u(s)=\Pi_{\ell} \tilde{u}(s)$ for all $s \geq 1$, $u_{[1, \infty)}, \tilde{u}_{[1, \infty)} \in B(M)$, and $u, \tilde{u}$ are solutions for some noise realizations $W$ and $\tilde{W}$ so $\Pi_{h} W=\Pi_{h} \tilde{W}$. These are precisely the conditions needed to apply the contractive estimates from section 13 .

This factorization states that drawing from $\mathbf{P}_{\infty}$ is equivalent, as far as either $u(t)$ or $\tilde{u}(t)$, is concerned, to drawing from $\mathbf{S}_{\infty}$ with probability $\rho_{\infty}$ and $\mathbf{P}_{\infty} \mathbf{R}_{n}$ with probability $\rho_{n-1}-\rho_{n}$. Of course, we have built in useful correlations between the two processes. Also notice that $\mathbf{P}_{\infty}$ appears on the left hand side, so the factorization can be iterated.

\subsection{Estimates on the $\rho$ 's}

The following estimates on the $\rho$ are the principle information needed to prove the exponential mixing, other that the Lyapunov structure which will be described in the next section. The first estimate is enough to imply mixing. The fact that the spacing between the $\rho$ 's decays exponentially, combined with the exponential tails of the return time to the set $\mathbf{C}$ defined in the following section, give the exponential mixing rate.

Lemma 15.2. In the setting of Theorem 8 , let $B(M)$ be the set used to define $\bar{B}_{[0, n)}$ in the previous section. For any $M_{0}>0$ the following estimates hold for all $M$ large enough:

1. There exists a positive constant $\rho_{\infty}^{*}$, depending on $M$ and $M_{0}$, so that

$$
\inf _{u_{0}^{(i)}: V\left(u_{0}^{(i)}\right) \leq M_{0}} \rho_{\infty}\left(u_{0}^{(1)}, u_{0}^{(2)}\right) \geq \rho_{\infty}^{*}>0
$$

2. There exist positive constants $K_{1}$ and $\gamma_{1}$, also depending on $M$ and $M_{0}$, so that for all $u_{0}^{(i)}, i=1,2$ with $V\left(u_{0}^{(i)}\right) \leq M_{0}$,

$$
\rho_{n}\left(u_{0}^{(1)}, u_{0}^{(2)}\right)-\rho_{n+1}\left(u_{0}^{(1)}, u_{0}^{(2)}\right) \leq K_{1} \exp \left(-\gamma_{1} n\right) .
$$


The proof of this lemma will be given in section 15.7 .

\subsection{Consequences of the Lyapunov Structure}

We now make a modification in the presentation relative to [Mat02c] which is greater than notational (but still mainly cosmetics). We want to iterate the expansion (29). However we will only have nice control over the $\rho_{n}$ 's for $u_{0}, \tilde{u}_{0}$ in a particular subset of the phase space. Hence, we modify the expansion to include the steps needed to return to this subset.

As already mentioned under Assumption 4, a lemma analogous to Lemma 10.1 holds for the Lyapunov function $V$. From this it is straight forward that there exists an $\alpha \in(0,1)$ so that $\mathbb{E}\left\{V(u(t+1)) \mid \mathcal{F}_{t}\right\} \leq \alpha V(u(t))+C_{1}$. Hence, if we define $\mathbf{V}(u, \tilde{u})=V(u)+V(\tilde{u})$ then

$$
\mathbb{E}\left\{\mathbf{V}(u(t+1), \tilde{u}(t+1)) \mid \mathcal{F}_{t}\right\} \leq \alpha \mathbf{V}(u(t), \tilde{u}(t))+2 C_{1} .
$$

We define the set $\mathbf{C}=\left\{(u, \tilde{u}): \mathbf{V}(u, \tilde{u}) \leq \frac{4 C_{1}}{\alpha}\right\}$ and the stopping time

$$
\tau_{C}=\inf \{s \geq 0: s \in \mathbb{N} ;(u(s), \tilde{u}(s)) \in \mathbf{C}\} .
$$

Lastly set $M_{0}=\frac{4 C_{1}}{\alpha}$ and fix $M$, from the previous two sections so the conclusions of Lemma 15.2 hold. The importance of this choice of $M_{0}$ and hence the definition of $\mathbf{C}$ are given by the following result.

Lemma 15.3. Under Assumption 4, $\mathbb{P}\left\{\tau_{\mathbf{C}}\left(u_{0}, \tilde{u}_{0}\right)>n\right\} \geq K_{0} \gamma_{0}^{n} \mathbf{V}\left(u_{0}, \tilde{u}_{0}\right)$ for any $\gamma_{0} \in(\alpha, 1)$ and some positive $K_{0}=K_{0}(\gamma)$.

Proof of Lemma 15.3: This result can be found many places. See for instance Lemma 11.3.9 of [MT93], Lemma 9.3 of [MSH02] or in the continuous time setting and in the context of the SNS Lemma 3.2 [EM01].

\subsection{Coupling: A New Representation of the Process}

We will define a new presentation of the chain using the factorization (29). First however, we modify the factorization slightly. In light of the previous section, the process $(u(t), \tilde{u}(t))$ returns to the set $\mathbf{C}$ infinitely often at integer times almost surely. Let $\mathbf{P}_{*}\left(u_{0}, \tilde{u}_{0}, \cdot\right)$ be the distribution of $\left(u_{\left[0, \tau_{C}\right]}, \tilde{u}_{\left[0, \tau_{C}\right]}, \eta_{\left[0, \tau_{C}\right]}\right)$ where $(u(0), \tilde{u}(0))=$ $\left(u_{0}, \tilde{u}_{0}\right)$. Then $\mathbf{P}_{*}(u(0), \tilde{u}(0), \cdot)$ is a probability measure on $\chi$ where

$$
\chi=\bigcup_{k=0}^{\infty} \mathbb{X}_{[0, k]} \times \mathbb{X}_{[0, k]} \times \Pi_{h} \Omega_{[0, k]} .
$$

The case $k=0$ is added to cover the situation when $\left(u_{0}, \tilde{u}_{0}\right) \in \mathbf{C}$ already. Since we only want to use the previous factorization for $\left(u_{0}, \tilde{u}_{0}\right) \in \mathbf{C}$, we redefine $\rho_{n}\left(u_{0}, \tilde{u}_{0}\right)=$ 0 for $\left(u_{0}, \tilde{u}_{0}\right) \notin \mathbf{C}$ and set $\mathbf{S}_{n}$ equal to the null measure for $\left(u_{0}, \tilde{u}_{0}\right) \notin \mathbf{C}$. Hence for $\left(u_{0}, \tilde{u}_{0}\right) \notin \mathbf{C}, \mathbf{r}_{1}=\mathbf{P}_{1}$ and all other $\mathbf{r}_{n}$ are then the null measure. The result is that for $\left(u_{0}, \tilde{u}_{0}\right) \neq \mathbf{C}$, the chain takes a step of length one with $u$ and $\tilde{u}$ stepping independently. 
Returning to the general case $\left(u_{0}, \tilde{u}_{0}\right) \in \mathbb{X} \times \mathbb{X}$. Defining $\mathbf{R}_{n *}=\mathbf{P}_{*} \mathbf{R}_{n}$, the factorization (29) can be rewritten

$$
\mathbf{P}_{\infty}\left(u_{0}, \tilde{u}_{0}, \cdot\right) \stackrel{\mathrm{M}}{=} \rho_{\infty} \mathbf{S}_{\infty}+\left[1-\rho_{\infty}\right] \sum_{n=1}^{\infty} \frac{\left[\rho_{n-1}-\rho_{n}\right]}{1-\rho_{\infty}} \mathbf{P}_{\infty} \mathbf{R}_{n *} .
$$

Again we have suppressed the dependence of the right hand side on the initial conditions. Defining

$$
\mathbf{R}_{\infty *}\left(u_{0}, \tilde{u}_{0}, \cdot\right)=\sum_{n=1}^{\infty} \frac{\left[\rho_{n-1}\left(u_{0}, \tilde{u}_{0}\right)-\rho_{n}\left(u_{0}, \tilde{u}_{0}\right)\right]}{1-\rho_{\infty}\left(u_{0}, \tilde{u}_{0}\right)} \mathbf{R}_{n *}\left(u_{0}, \tilde{u}_{0}, \cdot\right),
$$

we consider the chain $X_{n}=\left(x_{n}, \tilde{x}_{n}, \eta_{n}\right)$ on the state space $\bar{\chi}=\chi \cup\left(\mathbb{X}_{[0, k \infty)} \times \mathbb{X}_{[0, \infty)} \times\right.$ $\left.\Pi_{h} \Omega_{[0, \infty)}\right)$ given by taking steps from probability transition kernel

$$
\rho_{\infty}\left(u_{0}, \tilde{u}_{0}\right) \mathbf{S}_{\infty}\left(u_{0}, \tilde{u}_{0}, \cdot\right)+\left[1-\rho_{\infty}\left(u_{0}, \tilde{u}_{0}\right)\right] \mathbf{R}_{\infty *}\left(u_{0}, \tilde{u}_{0}, \cdot\right)
$$

We define

$$
t_{n}=\sum_{k=1}^{n-1}\left|x_{k}\right|=\sum_{k=1}^{n-1}\left|\tilde{x}_{k}\right|
$$

where $\left|x_{k}\right|$ is the length of the trajectory segment $x_{k} . t_{n}$ is the time passed in the physical PDE setting after $n$ steps of the chain have passed. Since the chain adds segments of random length on each step, $t_{n}$ is a random quantity. Similarly associated to $X_{n}$ is a trajectory $(u(t), \tilde{u}(t))$ of the SPDE. It is defined by $(u(t), \tilde{u}(t))=$ $\left(x_{n}\left(t-t_{n}\right), \tilde{x}_{n}\left(t-t_{n}\right)\right)$ where $t_{n}$ is the unique $t_{k}$ such that $t_{k} \leq t \leq t_{k}+\left|x_{k}\right|$. We will use both notations depending on which is the most convenient. We are, of course, only interested in $X_{n}$ through the step when $\left|x_{n}\right|=\infty$. This happens the first time a segment is drawn from $\mathbf{S}_{\infty}$. For reasons that will be clear, if they are not already, we refer to this as the "coupling time." We define the stopping time

$$
\tau=\inf \left\{n:\left|x_{n}\right|=\infty\right\}
$$

We pause for a second to notice some of the properties of the chain we have built. On the first step if $(u(0), \tilde{u}(0)) \notin \mathbf{C}$, it takes one step, adding a piece of trajectory of variable, integer length according to $\mathbf{P}_{*}(u(0), \tilde{u}(0), \cdot)$. Hence, at the end of this step, the system is in $\mathbf{C}$. Henceforward each step starts and ends in C. With probability $1-\rho_{\infty}$ the chain draws from $\mathbf{R}_{\infty * *}$. Each of these paths is of finite length. Their statistics are discussed below. With probability $\rho_{\infty}$ a path of infinite length is drawn from $\mathbf{S}_{\infty}$. After one unit of time, paths draw from $\mathbf{S}_{\infty}$ are, by construction, contained in $B(M)$. In addition by construction, they have norm at time one less than $M$, use the same $\eta$ increments, and agree on $\mathbb{X}_{\ell}$ for $t \geq 1$. Since at time one the norm is less than $M$, we have an a priori bound to the separation in the high modes. Thus, if $(v, \tilde{v}, \eta)$ is drawn according to $\mathbf{S}_{\infty}$, then from (28), $|v(t)-\tilde{v}(t)|_{\mathbb{X}}=\left|\Pi_{h} v(t)-\Pi_{h} \tilde{v}(t)\right|_{\mathbb{X}} \leq M e^{M} \exp \left(-\frac{1}{2} \gamma_{*} t\right)$. 


\subsection{The Heart of the Convergence Result}

We now show how the previous two sections quickly give the needed estimates to prove Theorem 8. For $\phi \in \mathcal{G}_{*}$ one has

$$
\begin{aligned}
\mathbb{E} \phi(u(t))-\phi(\tilde{u}(t)) & =\mathbb{E}[\phi(u(t))-\phi(\tilde{u}(t))]\left[\mathbf{1}_{t_{\tau}>\frac{t}{2}}+\mathbf{1}_{t_{\tau} \leq \frac{t}{2}}\right] \\
& \leq 2 \mathbb{P}\left\{t_{\tau}>\frac{t}{2}\right\}+M e^{M} \exp \left(-\frac{1}{4} \gamma_{*} t\right)
\end{aligned}
$$

The first term in the estimate follows from $\phi(u(t))-\phi(\tilde{u}(t))<2$ and $\mathbb{E} \mathbf{1}_{t_{\tau}>t / 2}=$ $\mathbb{P}\left\{t_{\tau}>t / 2\right\}$. The second term follows because for $t>2 t_{\tau}$ the system has been following a trajectory drawn from $\mathbf{S}_{\infty}$ for at least $t / 2$ units of time. Hence,

$$
\left|\Pi_{h} u(t)-\Pi_{h} \tilde{u}(t)\right|_{\mathbb{X}} \leq M e^{M} \exp \left(-\frac{1}{2} \gamma_{*} \frac{t}{2}\right)
$$

as noted in the previous paragraph. Next observe that $t_{\tau}\left(u_{0}, \tilde{u}_{0}\right)=\tau_{C}\left(u_{0}, \tilde{u}_{0}\right)+$ $t_{\tau}\left(u_{\tau_{C}}, \tilde{u}_{\tau_{C}}\right)$ where $t_{\tau}\left(u_{0}, \tilde{u}_{0}\right)$ and $t_{\tau}\left(u_{\tau_{C}}, \tilde{u}_{\tau_{C}}\right)$ means the stopping time starting from initial conditions $\left(u_{0}, \tilde{u}_{0}\right)$ and $\left(u_{\tau_{C}}, \tilde{u}_{\tau_{C}}\right)$ respectively. Hence,

$$
\mathbb{P}\left(t_{\tau}\left(u_{0}, \tilde{u}_{0}\right)>n\right) \leq \mathbb{P}\left(\tau_{C}\left(u_{0}, \tilde{u}_{0}\right)>\frac{n}{2}\right)+\sup _{\left(u_{0}^{\prime}, \tilde{u}_{0}^{\prime}\right) \in \mathbf{C}} \mathbb{P}\left(t_{\tau}\left(u_{0}^{\prime}, \tilde{u}_{0}^{\prime}\right)>\frac{n}{2}\right) .
$$

We know from Lemma 15.3 that $\mathbb{P}\left(\tau_{C}\left(u_{0}, \tilde{u}_{0}\right)>\frac{n}{2}\right)$ is exponentially decaying in $n$ with a constant which scales linearly with $\mathbf{V}\left(u_{0}, \tilde{u}_{0}\right)$. Hence, Theorem 8 would be proven. If we show that $\sup \mathbb{P}\left(t_{\tau}\left(u_{0}^{\prime}, \tilde{u}_{0}^{\prime}\right)>\frac{n}{2}\right)$ decays exponentially in $n$. This is done in the next section.

The proof of Corollary 15.1 follows from similar reasoning.

$$
\begin{aligned}
\mathbb{E} \phi(u(t))-\phi(\tilde{u}(t))=\mathbb{E}[ & \phi(u(t))-\phi(\tilde{u}(t))]\left[\mathbf{1}_{t_{\tau}>\frac{t}{2}}+\mathbf{1}_{t_{\tau} \leq \frac{t}{2}}\right] \\
\leq & \mathbb{E} R(u(t)) \mathbf{1}_{t_{\tau}>\frac{t}{2}}+\mathbb{E} R(\tilde{u}(t)) \mathbf{1}_{t_{\tau}>\frac{t}{2}} \\
& \quad+M e^{M} \exp \left(-\frac{1}{4} \gamma_{*} t\right)[1+\mathbb{E} R(u(t))+\mathbb{E} R(\tilde{u}(t))] \\
\leq[ & \left.\left(\mathbb{E}\left(R(u(t))^{q}\right)\right)^{\frac{1}{q}}+\left(\mathbb{E}\left(R(\tilde{u}(t))^{q}\right)\right)^{\frac{1}{q}}\right]\left[\mathbb{P}\left\{t_{\tau}>\frac{t}{2}\right\}\right]^{\frac{1}{p}} \\
& \quad+M e^{M} \exp \left(-\frac{1}{4} \gamma_{*} t\right)\left[1+\left(\mathbb{E} R(u(t))^{q}\right)^{\frac{1}{q}}+\left(\mathbb{E} R(\tilde{u}(t))^{q}\right)^{\frac{1}{q}}\right] .
\end{aligned}
$$

Hence and exponential bound on $\mathbb{P}\left\{t_{\tau}>\frac{t}{2}\right\}$ will also complete the proof of the Corollary.

\subsection{Moments of the Coupling Time}

We now complete the proof of Theorem 8 by providing exponential control of the moments of $t_{\tau}$. The missing pieces are the following lemma, which we will proven at the end of this section and some estimates on the $\rho$ 's given in the next section.

Lemma 15.4. There exist positive constants $\gamma_{2}$ and $K_{2}$ so that for all $\left(u_{0}, \tilde{u}_{0}\right) \in \mathbf{C}$, $\mathbb{E} \exp \left(\gamma_{2}\left|\mathbf{R}_{\infty *}\left(u_{0}, \tilde{u}_{0}\right)\right|\right) \leq \exp \left(K_{2}\right)$. Where $\left|\mathbf{R}_{\infty *}\left(u_{0}, \tilde{u}_{0}\right)\right|$ is the random variable distributed as the length of a segment drawn from $\mathbf{R}_{\infty *}\left(u_{0}, \tilde{u}_{0}, \cdot\right)$. 
Using this lemma we quickly finish the proof of Theorem 8. For any $a \in(0,1)$ and $\left(u_{0}, \tilde{u}_{0}\right) \in \mathbf{C}$

$$
\begin{aligned}
\mathbb{P}\left\{t_{\tau}\left(u_{0}, \tilde{u}_{0}\right)>n\right\} & =\mathbb{P}\left\{t_{\tau}>n ; \tau>a n\right\}+\mathbb{P}\left\{t_{\tau}>n ; \tau \leq a n\right\} \\
& \leq \mathbb{P}\{\tau>a n\}+\mathbb{P}\left\{t_{\tau}>n ; \tau \leq a n\right\} \\
& \leq\left(1-\rho_{\infty}^{*}\right)^{\lfloor a n\rfloor}+e^{-\left(\gamma_{2}-K_{2} a\right) n}
\end{aligned}
$$

where $\gamma_{2}$ and $K_{2}$ are the constants from Lemma 15.4 and $\rho_{\infty}^{*}$ from Lemma 15.2. The first estimate follows because on each step of the chain there is at least a $\rho_{\infty}^{*}$ chance of drawing from $\mathbf{S}_{\infty}$. Accepting the second estimate for a moment, choosing any $a \in\left(0,1 \wedge \frac{\gamma_{2}}{K_{2}}\right)$ gives exponential decay and completes the proof.

To see the second estimate, observe that from Lemma 15.4 and the fact that $\left(u_{0}, \tilde{u}_{0}\right) \in \mathbf{C}, \mathbb{E} \exp \left(\gamma_{2} \sum_{k=1}^{a n}\left|x_{k}\right|\right) \leq \exp \left(a n K_{2}\right)$. Hence one has

$$
\mathbb{P}\left\{t_{\tau}>n ; \tau \leq a n\right\} \leq \mathbb{P}\left\{\sum_{k=1}^{a n}\left|x_{k}\right|>n ;\right\} \leq e^{-\left(\gamma_{2}-K_{2} a\right) n}
$$

Proof: Proof of Lemma 15.4 Let $\left|\mathbf{R}_{n *}\right|$ be the random variable distributed as the length of a trajectory drawn from $\mathbf{R}_{n *}\left(u_{0}, \tilde{u}_{0}, \cdot\right)$. In what follows, we suppress the dependence on the initial conditions $\left(u_{0}, \tilde{u}_{0}\right)$ of the $\rho$ 's and the transition kernels as we always consider the same initial conditions.

Define the random variable $\zeta$ as follows by

$$
\zeta=k \text { with probability for } k \in\{1,2, \ldots\} \frac{\rho_{k-1}-\rho_{k}}{1-\rho_{\infty}} .
$$

Then $\left|\mathbf{R}_{\infty *}\right|$ is distributed as $\left|\mathbf{R}_{\zeta *}\right|$. Hence, we have

$$
\begin{aligned}
\mathbb{P}\left\{\left|\mathbf{R}_{\infty *}\right|>n\right\} & =\mathbb{P}\left\{\left|\mathbf{R}_{\zeta *}\right|>n ; \zeta>\frac{n}{2}\right\}+\mathbb{P}\left\{\left|\mathbf{R}_{\zeta *}\right|>n ; \zeta \leq \frac{n}{2}\right\} \\
& \leq \mathbb{P}\left\{\zeta>\frac{n}{2}\right\}+\mathbb{P}\left\{\left|\mathbf{R}_{\zeta *}\right|>n ; \zeta \leq \frac{n}{2}\right\} .
\end{aligned}
$$

The first term decays exponentially by the second part of Lemma 15.2. This leaves only the last term.

$$
\mathbb{P}\left\{\left|\mathbf{R}_{\zeta *}\right|>n ; \zeta \leq \frac{n}{2}\right\} \leq \sum_{k=1}^{\frac{n}{2}} \mathbb{P}\left\{\left|\mathbf{R}_{k *}\right|>n\right\} \frac{\rho_{k-1}-\rho_{k}}{1-\rho_{\infty}}
$$

Notice that $\left|\mathbf{R}_{k *}\right|$ is $k$ plus the time to return to $\mathbf{C}$ starting from $(u(k), \tilde{u}(k))$. Using Lemma 15.3 and that by definition $\left[\rho_{k-1}-\rho_{k}\right] \mathbf{R}_{k}=\mathbf{r}_{k}$ produces

$$
\begin{aligned}
& \mathbb{P}\left\{\left|\mathbf{R}_{\zeta *}\right|>n ; \zeta \leq \frac{n}{2}\right\} \\
& \leq \frac{1}{1-\rho_{\infty}} \sum_{k=1}^{\frac{n}{2}} \int \mathbb{P}\left\{\tau_{C}(u(k), \tilde{u}(k))>n-k\right\} \mathbf{R}_{k}(d u, d \tilde{u})\left[\rho_{k-1}-\rho_{k}\right] \\
& \quad \leq \frac{K}{1-\rho_{\infty}} \sum_{k=1}^{\frac{n}{2}} \gamma^{n-k} \int \mathbf{V}(u(k), \tilde{u}(k)) \mathbf{r}_{k}\left(u_{0}, \tilde{u}_{0}, d u_{[0, k)}, d \tilde{u}_{[0, k)}\right) .
\end{aligned}
$$


By the definition of $\mathbf{r}_{k}$ one sees that for any measurable set $A, \mathbf{P}_{k}\left(u_{0}, \tilde{u}_{0}, A\right) \geq$ $\mathbf{r}_{k}\left(u_{0}, \tilde{u}_{0}, A\right)$. Since $\mathbf{V}$ is positive, we have

$$
\begin{aligned}
\int \mathbf{V}(u(k), \tilde{u}(k)) \mathbf{r}_{k}\left(d u_{[0, k)}, d \tilde{u}_{[0, k)}\right) & \leq \int \mathbf{V}(u(k), \tilde{u}(k)) \mathbf{P}_{k}\left(d u_{[0, k)}, d \tilde{u}_{[0, k)}\right) \\
& =\mathbb{E}\left\{\mathbf{V}(u(k), \tilde{u}(k)) \mid(u(0), \tilde{u}(0))=\left(u_{0}, \tilde{u}_{0}\right)\right\} \\
& \leq K^{\prime \prime} \text { since }\left(u_{0}, \tilde{u}_{0}\right) \in \mathbf{C} .
\end{aligned}
$$

The uniform bound on the integral used to obtain the last estimate comes from a lemma controlling $\mathbf{V}$ completely analogous to Lemma 10.1 about the energy of the SNS. It can be found in many places. It is simply integrating up the Lyapunov estimate in time. See for instance Lemma 9.3 of [MSH02] or Lemma 11.3.9 of [MT93]. Continuing, one has

$$
\mathbb{P}\left\{\left|\mathbf{R}_{\zeta *}\right|>n ; \zeta \leq \frac{n}{2}\right\} \leq \frac{K^{\prime}}{1-\rho_{\infty}} \sum_{k=1}^{\frac{n}{2}} \gamma_{0}^{n-k} \leq \frac{K^{\prime}}{1-\rho_{\infty}} \gamma_{0}^{\frac{n}{2}}
$$

\subsection{Proof of Lemma 15.2}

Proof of Lemma 15.2: The details of a similar argument are on page 452 of [Mat02c]. We begin with the first statement. For any $M>0$ and $A \subset \mathbb{X}_{\ell}$, define

$$
\begin{aligned}
Q_{t}^{\eta}\left(u_{0}, A ; M\right) & =\mathbb{P}\left(\Pi_{\ell} u(t) \in A ; V(u(t)) \leq M \mid u(0)=u_{0}, \mathcal{F}_{[0, t]}^{\eta}\right) \\
& =\mathbb{E}\left\{\mathbf{1}_{A}\left(\Pi_{\ell} u(t)\right) \mathbf{1}_{V(u(t)) \leq M} \mid u(0)=u_{0}, \mathcal{F}_{[0, t]}^{\eta}\right\} .
\end{aligned}
$$

Since $\sup _{u_{0}: V\left(u_{0}\right) \leq M_{0}} \mathbb{E}\{V(u(t))\}<\infty$, for all $M$ sufficiently large one has

$$
\inf _{u_{0}: V\left(u_{0}\right) \leq M_{0}} \mathbb{P}\left\{V(u(t))<M^{\frac{1}{2}}\right\}>1-\delta / 10 .
$$

Hence there exist a $\Omega^{\prime \prime} \subset \Omega^{\prime}$ so $\mathbb{P}\left(\eta \in \Omega^{\prime \prime}\right)>\delta / 2$ and for all $\eta \in \Omega^{\prime \prime}$ and $u_{0}^{(i)} \in \mathbb{X}, i=$ 1,2 , with $V\left(u_{0}^{(i)}\right) \leq M_{0}$, one has $\left\|Q_{t}^{\eta}\left(u_{0}^{(1)}, \cdot ; M^{\frac{1}{2}}\right)-Q_{t}^{\eta}\left(u_{0}^{(2)}, \cdot ; M^{\frac{1}{2}}\right)\right\|_{T V}<1-\delta / 2$. Then

Now define $\Gamma^{\eta}\left(u_{0}, \tilde{u}_{0}, \cdot\right)=Q_{[0, \infty)}^{\eta}\left(u_{0}, \cdot ; u \in B(M)\right) \wedge Q_{[0, \infty)}^{\eta}\left(\tilde{u}_{0}, \cdot ; u \in B(M)\right)$.

$$
\rho_{\infty}\left(u_{0}, \tilde{u}_{0}\right) \geq \frac{\delta^{2}}{4} \inf _{\substack{u_{0}, \tilde{u}_{0} \in \mathbb{X}, \Pi_{\ell} u_{0}=\Pi_{\ell} \tilde{u}_{0} \\ V\left(u_{0}\right), V\left(\tilde{u}_{0}\right) \leq M^{\frac{1}{2}}}} \mathbb{E} \Gamma^{\eta}\left(u_{0}, \tilde{u}_{0}, \Pi_{\ell} \mathbb{X}_{[0, \infty)} \times \Pi_{\ell} \mathbb{X}_{[0, \infty)} \times \Pi_{\ell} \Omega_{[0, \infty)}\right)
$$

Since (28) holds in this setting, the exact same calculations as in the proof of the second half of Lemma 14.1 hold producing an estimate identical to Lemma 14.2 with $B_{n}^{\prime}$ replaced by $\{u \in B(M)\}$ and valid for $u_{0}, \tilde{u}_{0}$ with $V\left(u_{0}\right), V\left(\tilde{u}_{0}\right) \leq M^{\frac{1}{2}}$. Combining this estimate with Lemma B.1, we obtain for any $p>1$

$$
\inf _{\substack{u_{0}, \tilde{u}_{0} \in \mathbb{X}, \Pi_{\ell} u_{0}=\Pi_{\ell} \tilde{u}_{0} \\ V\left(u_{0}\right), V\left(\tilde{u}_{0}\right) \leq M^{\frac{1}{2}}}} \mathbb{E} \Gamma^{\eta}\left(u_{0}, \tilde{u}_{0}, \Pi_{\ell} \mathbb{X}_{[0, \infty)} \times \Pi_{\ell} \mathbb{X}_{[0, \infty)} \times \Pi_{\ell} \Omega_{[0, \infty)}\right) \geq\left[1-\frac{1}{p}\right] \frac{C(M)^{\frac{p}{p-1}}}{p^{\frac{1}{p-1}} D_{*}^{p}} .
$$


where $C(M)=\inf _{u_{0}: V\left(u_{0}\right) \leq M^{\frac{1}{2}}} \mathbb{E} Q_{[0, \infty)}^{\eta}\left(u_{0}, \Pi_{\ell} \mathbb{X}_{[0, \infty)} \times \Pi_{\ell} \mathbb{X}_{[0, \infty)} \times \Pi_{\ell} \Omega_{[0, \infty)} ; u \in B(M)\right)$ and $D_{*}$ is the constant defined analogously to (26).

Notice that

$$
\mathbb{E} Q_{[0, \infty)}^{\eta}\left(u_{0}, \Pi_{\ell} \mathbb{X}_{[0, \infty)} \times \Pi_{\ell} \mathbb{X}_{[0, \infty)} ; u \in B(M)\right)=\mathbb{P}\left\{u_{[0, \infty)} \in B(M) \mid u(0)=u_{0}\right\}
$$

Hence for $M$ sufficiently large, for all $u_{0}$ with $V\left(u_{0}\right) \leq M^{\frac{1}{2}}$ there exists a set $\Omega_{0}^{\prime \prime \prime} \subset$ $\Pi_{\ell} \Omega$ so that $\mathbb{P}\left(\eta \in \Omega_{0}^{\prime \prime \prime}\right)>1-\delta / 100$ and for all $\eta \in \Omega_{0}^{\prime \prime \prime} P_{[0, \infty)}^{\eta}\left(u_{0}, B(M)\right)>1-\delta / 100$. Hence $C(M) \geq(1-\delta / 100)^{2}$. This completes the first claim.

Now consider the second claim. Setting $\mathbb{Y}_{n}=\Pi_{\ell} \mathbb{X}_{[0, n)} \times \Pi_{\ell} \mathbb{X}_{[0, n)} \times \Pi_{\ell} \Omega_{[0, n)}$, notice that $\rho_{n-1}\left(u_{0}, \tilde{u}_{0}\right)-\rho_{n}\left(u_{0}, \tilde{u}_{0}\right)=\mathbf{r}_{n}\left(u_{0}, \tilde{u}_{0}, \mathbb{Y}_{n}\right)=\left[\mathbf{P}_{1} \mathbf{s}_{n-1}-\mathbf{s}_{n}\right]\left(u_{0}, \tilde{u}_{0}, \mathbb{Y}_{n}\right)$. From this we see that $\rho_{n-1}-\rho_{n}$ is the probability of drawing from $\mathbf{s}_{n-1}$ but not from $\mathbf{s}_{n}$. There are two ways this can happen. First the trajectory can leave the set $B(M)$ between time $n-1$ and $n$. This probability is exponentially small in $n$ by the construction of $B(M)$ and the estimate in (27). The second way is to draw from the part of distribution contained in $B(M)$ between time $n-1$ and $n$ but not in the common part of the two $Q^{\eta}$ distributions. Over $[0, n-1]$ trajectories $(u, \tilde{u}, \eta)$ are drawn from $\mathbf{s}_{n-1}$. Hence almost every trajectory has the properties that $\Pi_{\ell} u_{[1, n-1]}=\Pi_{\ell} \tilde{u}_{[1, n-1]}$ and both are in $\Pi_{[0, n-1]} B(M)$. The contractive property derived analogously to $(25)$ then implies, $|u(n-1)-\tilde{u}(n-1)|_{\mathbb{X}} \leq$ $M e^{M} e^{-\frac{1}{2} \gamma_{*}(n-1)}$. Let $\bar{B}_{[n-1, n]}\left(u_{[0, n-1)}, \tilde{u}_{[0, n-1)}, \eta_{[0, n-1)}\right)$ be the paths in $\Pi_{\ell} \mathbb{X}_{[n-1, n]} \times$ $\Pi_{\ell} \mathbb{X}_{[n-1, n]} \times \Pi_{\ell} \Omega_{[n-1, n]}$ so that when added to $\left(u_{[0, n-1]}, \tilde{u}_{[0, n-1]}, \eta_{[0, n-1]}\right)$ the resulting path $\left(u_{[0, n]}, \tilde{u}_{[0, n]}, \eta_{[0, n]}\right)$ is such that $u_{[0, n]}, \tilde{u}_{[0, n]} \in \Pi_{[0, n]} B(M)$. (As before the part of the trajectory in $\Pi_{h} \mathbb{X}_{[n-1, n]}$ has to be reconstructed with the aid of $\Phi$.) Hence $Q_{[0,1]}^{\eta}\left(u(n-1), \cdot ; \bar{B}_{[n-1, n]}\right)$ and $Q_{[0,1]}^{\eta}\left(\tilde{u}(n-1), \cdot ; \bar{B}_{[n-1, n]}\right)$ where $\bar{B}_{[n-1, n]}=$ $\bar{B}_{[n-1, n]}\left(u_{[0, n-1]}, \tilde{u}_{[0, n-1]}, \eta_{[0, n-1]}\right)$, are the two distributions which will be used to draw the next unit length step. Thus the term we need to control is

$$
\begin{array}{r}
\frac{1}{2} \mathbb{E}\left\|Q_{[0,1]}^{\eta}\left(u(n-1), \cdot ; \bar{B}_{[n-1, n]}\right)-Q_{[0,1]}^{\eta}\left(\tilde{u}(n-1), \cdot ; \bar{B}_{[n-1, n]}\right)\right\|_{T V} \\
\leq \mathbb{E}\left(\mathbb { E } \left\{\left[\frac{d Q_{[0,1]}^{\eta}(u(n-1), \cdot)}{d Q_{[0,1]}^{\eta}(\tilde{u}(n-1), \cdot)}-1\right]^{2} \mathbf{1}_{\left.\left.\bar{B}_{[n-1, n]} \mid \mathcal{F}^{\eta}\right\}\right)^{\frac{1}{2}}}\right.\right. \\
\leq\left(\exp \left(K e^{-\frac{1}{2} \gamma_{*} n}\right)-1\right)^{\frac{1}{2}}
\end{array}
$$

The main estimate comes from the last estimate of Lemma A.1 applied on the measure conditioned on a fixed $\eta$ path. The estimate $\exp \left(K e^{-\frac{1}{2} \gamma_{*} n}\right)$ is the estimate on the constant $D_{*}$ used in Lemma A.1. This estimate is a consequence of the contractive property noticed above use to estimate the difference term

$$
\exp \left(\int_{n-1}^{n} \frac{1}{\sigma_{\min }^{2}}\left|\Pi_{\ell} G(u(t))-\Pi_{\ell} G(\tilde{u}(t))\right|_{\mathbb{X}}^{2} d t\right)
$$

in a fashion analogous to (26). 


\section{Other Examples}

The general assumptions used in the previous example are general enough to cover a number of SPDEs of interest. A natural second example where all of our analysis applies is the stochastically forced Cahn-Allen/Ginsburg-Landau equation

$$
d u(x, t)=\left[\nu \Delta u+u-u^{3}\right] d t+d W(x, t)
$$

where $W(x, t)=\sum_{\mathcal{K}} e_{k}(x) \sigma_{k} \beta(t), \beta_{k}$ are independent standard Brownian motions, $\sigma_{k}$ are positive constants and $e_{k}$ are the elements of the real Fourier basis

$$
\{1, \sin (2 \pi x), \cos (2 \pi x), \sin (4 \pi x), \cos (4 \pi x), \cdots\} .
$$

See [BM03, EL02] for the verification of the assumptions. (Note that text assumes that $\mathbb{X}_{h}$ is not forced; however, the verification of the assumptions given there allows one to cover that case with the theorems provided in this text.) One uses the Lyapunov structure $V(u)=U(u)=|u|_{\mathbb{L}^{2}}^{2}+|\nabla u|_{\mathbb{L}^{2}}^{2}$. That case is also analyzed in [Hai02]. In that reference, the strong contractive nature is used to get an exponential mixing rate uniform in the initial data. This is because the time for the initial return center of the phase space does not depend on the initial state; this is not the case in the SNS equation. This holds because one can estimate the time $\tau_{C}$ uniformly in the initial data. Hence from (35), one sees that the mixing time can be estimated independent of the initial data. This is made explicitly in the theorems in [Mat02c]. Another noteworthy feature of the analysis in [Hai02] is that a change of measure is made in the low modes to steer all of the modes together only asymptotically. In contrast to the presentation given here where the $\ell$ variable is made to be equal for all moments of time after $t=1$ and the $h$ variable converges asymptotically. The method in [Hai02] appears to be simpler to construct while the method exposed here gives convergence in a slightly stronger topology.

In [EL02] other examples are given, all but the stochastic Kuramoto-Sivashinsky, fits directly in the framework given here. The stochastic Kuramoto-Sivashinsky equation requires localization ideas not based on a straight forward Lyapunov function. The details are explained fully in [EL02].

\section{True Hypoellipticity and the Cascade of Randomness}

It is reasonable to ask if the results given in Theorem 3 or Theorem 7 are sharp. Does ergodicity require forcing all of the modes below the scale specified by the balance between energy influx and dissipation? The assumption for the second part of Theorem 3 is an ellipticity assumption about the dynamics in the typically unstable directions. Equivalently viewed from the Memory/Gibbsian dynamics point of view, it means that the reduced system with memory (9) is elliptic.

While there is no complete proof, there are a number of results which seem to imply that much weaker conditions are sufficient. They all describe the dynamics in a hypoelliptic setting; the case where all of the typically unstable degrees of freedom are not forced directly. In this setting, ergodicity and mixing require that the nonlinearity transfer the randomness to other degrees of freedom. 
The first result given below proves the ergodicity of an arbitrary Galerkin approximation of (4) under very weak assumptions. Under similar assumptions, the second result says that the full PDE has a transition density whose finite dimensional marginals have a density with respect to Lebesgue measure.

A third result by [Rom02] proves the geometric ergodicity of the Galerkin projections of the three dimensional SNS equations. This was expected as the structure shares the needed structure with the two dimensional problem. What was extremely interesting and novel in that paper was the proof that the system was globally controllable. A fourth result found in [AS03] shows that the full two and three dimensional SNS equations are controllable in the sense that one can steer them so that any finite number of modes take specified values. This is very similar in spirit to Theorem 10 where only projections of the transition measure are shown to have a density. The techniques used to prove the control results in [Rom02] and [AS03] seem to use the same important observation. Namely that the off-diagonal nature of the nonlinearity leaves the system globally consolable even though its nonlinearity is even powered. We refer the reader to [Rom02] and [AS03] for the precise statement of the results.

As we will undertake direct calculations, it is simpler to work in a real basis of $L^{2}\left(\mathbb{T}^{2}\right)$. For this reason we switch our forcing to the form

$$
W(x, t)=\sum_{k \in \mathcal{K}^{\cos }} \sigma_{k}^{\cos } \cos (k \cdot x) b_{k}(t)+\sum_{k \in \mathcal{K}^{\sin }} \sigma_{k}^{\sin } \sin (k \cdot x) B_{k}(t)
$$

where $B_{k}$ and $b_{k}$ are independent real Brownian motions with variance one, $\sigma_{k}^{\text {cos }}$, $\sigma_{k}^{\text {sin }}$ are positive real constants, and $\mathcal{K}^{\text {cos }}, \mathcal{K}^{\text {sin }}$ are subsets of $\mathbb{Z}_{*}^{2} \stackrel{\text { def }}{=}\left\{j=\left(j_{1}, j_{2}\right) \in\right.$ $\left.\mathbb{Z}^{2}: j_{2} \geq 0,|j|>0\right\}$. We need only to consider $\mathbb{Z}_{*}^{2}$ as the reality of the vorticity allows one to restrict to wave number in the upper half plane and we have assumed the absence of a mean flow. (Note: In [EM01] the sums were restricted too much, however this does not effect any of the bracket calculations made and the results hold true.)

We now define two sequences of subsets of $\mathbb{Z}^{2}$ which capture how the randomness spreads from one degree of freedom to the next. Define $\mathcal{K}_{0}=\mathcal{Z}_{0}=\mathcal{K}^{\cos } \cap \mathcal{K}^{\text {sin }}$. Next define

$$
\begin{array}{r}
\mathcal{Z}_{n}=\mathcal{Z}_{n-1} \cup\left\{k \in \mathbb{Z}_{*}^{2}: k \in\{\ell+j, \ell-j, j-\ell\} \text { with } j \in \mathcal{Z}_{0}, \ell \in \mathcal{Z}_{n-1}\right. \\
\text { and } \left.\ell^{\perp} \cdot j \neq 0,|j| \neq|\ell|\right\}
\end{array}
$$

and fixing some positive integer $N$ define

$$
\begin{aligned}
& \mathcal{K}_{n}^{N}=\mathcal{K}_{n-1}^{N} \cup\left\{k \in \mathbb{Z}_{*}^{2}: k \in\{\ell+j, \ell-j, j-\ell\} \text { with } j, \ell \in \mathcal{K}_{n-1}^{N}\right. \\
&\text { and } \left.\ell^{\perp} \cdot j \neq 0,|j| \neq|\ell|,|\ell-j|<N,|\ell+j|<N\right\}
\end{aligned}
$$

and finally $\mathcal{Z}_{\infty}=\cup \mathcal{Z}_{n}$ and $\mathcal{K}_{\infty}^{N}=\cup \mathcal{K}_{n}^{N}$. The two sets track the cascade of randomness out to the unforced modes. The farther along the chain which a mode first enters the sequence of sets, the less the random variation will be felt in that coordinate. 
Theorem 9 below will state its assumptions in terms of $\mathcal{K}_{\infty}^{N}$ whereas Theorem 10 will use $\mathcal{Z}_{\infty}$. It is likely that for a given $\mathcal{K}^{\text {cos }}$ and $\mathcal{K}^{\text {sin }}$ that $\mathcal{Z}_{\infty}=\cup_{N} \mathcal{K}_{\infty}^{N}$ (one direction is clear) however proof is not immediately obvious. Furthermore, a sketch of Theorem 10, under the same assumptions as Theorem 9, is given in [MP03]. Hence, we do not think there is any real significant difference between the two sets.

The first result we state gives exponentially mixing for the order $N$ Galerkin approximation of (4) with forcing of the form (38) provided an algebraic condition on the wave numbers forced, given in terms of $\mathcal{K}_{\infty}^{N}$, is satisfied. By the Galerkin approximation of order $N$, we mean the finite system of coupled ODEs obtained by setting to zero, for all time, any Fourier mode with $|k| \geq N$. This approximation returns us to the setting of standard hypoelliptic SDE in $\mathbb{R}^{d}$. Using a weak version of Höromander's sum of squares theorem (cf. [KS84, Nor86, Be195]), it was shown that the diffusion has a smooth $C^{\infty}$ density. Then, using some standard Markov chain theory for a Harris chain with a Foster-Lyapunov function, one obtains exponential mixing.

Theorem 9. [EM01] Consider the order $N$ Galerkin approximation of the vorticity equation (4). Assume that $\mathcal{K}_{\infty}^{N}=\left\{k \in \mathbb{Z}_{*}^{2}:|k|<N\right\}$. Denoting the solution by $\omega^{N}$, one has the following mixing result.

If $\omega_{0}^{N}$ and $\tilde{\omega}_{0}^{N}$ are two initial conditions then for any $p \geq 1$ there exist positive constants $B=B(p)$ and $\gamma=\gamma(p)$ so that

$$
\begin{aligned}
\left\|P_{t}\left(\omega_{0}^{N}, \cdot\right)-P_{t}\left(\tilde{\omega}_{0}^{N}, \cdot\right)\right\|_{T V} \leq\left\|P_{t}\left(\omega_{0}^{N}, \cdot\right)-P_{t}\left(\tilde{\omega}_{0}^{N}, \cdot\right)\right\|_{V_{p}} \\
\\
\leq B\left[1+\left|\omega_{0}^{N}\right|^{2 p}+\left|\tilde{\omega}_{0}^{N}\right|^{2 p}\right] e^{-\gamma t}
\end{aligned}
$$

Here $\|\cdot\|_{T V}$ is the total variation norm on signed measures and $\|\cdot\|_{V(p)}$ is the weighted variational norm defined by

$$
\left\|P_{t}\left(\omega_{0}^{N}, \cdot\right)-P_{t}\left(\tilde{\omega}_{0}^{N}, \cdot\right)\right\|_{V_{p}} \stackrel{\text { def }}{=} \sup _{\phi \in \mathcal{V}_{p}} \mathbb{E} \phi\left(\omega^{N}(t)\right)-\mathbb{E} \phi\left(\tilde{\omega}^{N}(t)\right)
$$

with $\mathcal{V}_{p}=\left\{\right.$ measurable $\phi$ with $\left.|\phi(x)| \leq 1+|x|^{2 p}\right\}$. Taking $\tilde{\omega}_{0}^{N}$ distributed as the invariant measure, one obtains exponential convergence to the invariant measure and uniqueness of the invariant measure.

To make this theorem interesting, we need some examples of conditions on $\mathcal{K}^{\text {cos }}$ and $\mathcal{K}^{\text {sin }}$ so that it applies. The following Lemma gives simple conditions under which the previous and next theorems hold.

Lemma 17.1. [EMO1, MP03]

- If $\{(0,1),(1,1)\}$ or $\{(1,0),(1,1)\} \subset \mathcal{K}^{\cos } \cap \mathcal{K}^{\sin }$ then $\mathcal{Z}_{\infty}=\mathbb{Z}^{2}$ and $\mathcal{K}_{\infty}^{N}=\{k \in$ $\left.\mathbb{Z}_{*}^{2}:|k|<N\right\}$ for any $N$.

- Let $M, K \in \mathbb{N}$ with $M, K>2$ and $|M-K|>2$. Then if

$$
\{(M+1,0),(M, 0),(0, K+1),(0, K)\} \subset \mathcal{Z}_{0}
$$

then $\mathcal{Z}_{\infty}=\mathbb{Z}^{2}$. If in addition $M, K<N-1$ then $\mathcal{K}_{\infty}^{N}=\left\{k \in \mathbb{Z}_{*}^{2}:|k|<N\right\}$. 
This gives only two examples of types of forcing which are sufficiently distributed to ensure ergodicity. Many others choices are possible. The author thanks A. Majda and P. Constantin for stimulating conversations which pushed him to verify the second part of Lemma 17.1. It provides an example of forcing which allows one to observe both the energy and enstrophy cascade which are present in two dimensional fluid systems. Of course, the most interesting question would be to make some qualitative statement connecting this cascade of probability with the dynamics.

A theorem similar to Theorem 9, but for the three dimensional Galerkin approximation, is proven in [Rom02]. There he proves even more; he shows that the system is actually globally controllable. This very interesting fact hinges on the observation that because the nonlinearity is off-diagonal in Fourier space; and hence, the system has the good properties of systems with odd powered polynomials nonlinearities (see [Jur97]).

Theorem 9 gives a strong indication that a similar theorem holds for the full PDE; however, a proof currently alludes the community. The following theorem shows that at least one of the needed ingredients persists for the full infinite dimensional vorticity equation.

Defining

$$
S_{\infty}=\operatorname{Span}\left(\left\{\sin (k \cdot x): k \in \mathcal{Z}_{\infty} \cup \mathcal{Z}_{0}^{\cos }\right\} \cup\left\{\cos (k \cdot x): k \in \mathcal{Z}_{\infty} \cup \mathcal{Z}_{0}^{\sin }\right\}\right)
$$

we have the following density result for the finite dimensional marginals of equation (4).

Theorem 10. [MP03] For any $t>0$ and any finite dimensional subspace $S$ of $S_{\infty}$, the law of the orthogonal projection $\Pi_{S} \omega(t, \cdot)$ of $\omega(t, \cdot)$ onto $S$ is absolutely continuous with respect to the Lebesgue measure on $S$.

This of course is not enough to prove ergodicity. It addresses only the first part of Assumption 2.

\section{Open Questions}

A number of open questions have been mentioned in the text. Here we collect them and add a few more.

1. Extend the ergodic results to the case when all of the determining modes are not forced. The results on the ergodicity of the Galerkin approximation suggest strongly that full PDE is ergodic under weaker assumptions than Theorem 3. Theorem 9 gives and indication what the proper assumptions should be. The results on the existence of densities for the projection of transition densities and the controllability of a finite number of variables gives strong evidence that nothing surprising happens in the full PDE.

2. Prove (or disprove) that even when the forcing has spatial Fourier modes which decay super-exponentially, the solution still decays only exponentially in $|k|$. Prove (or disprove) that this decay rate does not fluctuate with time in the stationary state. 
3. Related to the previous: "What is the natural topology of the transition density of the Markov process defined by the SNS ?"

4. Extend Theorem 3 to the full space. The case of bounded domains in the same as the periodic case. However the full space requires some additional ideas, if not completely different ones.

5. Understand better the $\nu \rightarrow 0$ limit. In a recent preprint [Kuk03] explores this limit for one choice of forcing. However, the choice of scaling produces a deterministic limit which is the less interesting case and does not correspond to the traditional view of turbulence. In all cases, there remain many interesting question concerning the structure of the limiting solutions and the limit when other types of forcing are used.

6. Make progress in the three dimensional problem. Unless a breakthrough is made in the deterministic three dimensional problem, this would likely require other methods. The methods used here proceed in a pathwise manner in the high $k$ and, hence, can do no better than the deterministic theory. In particular, the estimates used to get contraction of the high $k$ are similar to those used to prove uniqueness of solutions. Recently Da Prato and Debussche have show that by a selection principle one can build a stochastic process associated to the 3D problem and that this process under certain conditions has a unique invariant measure. Unfortunately the conditions on the forcing require it to have algebraic decay in $|k|$.

\section{Acknowledgments}

I am indebted to my collaborators Yuri Bakhtin, Weinan E, Ya. Sinai, Toufic Suidan, Andrew Stuart for both their hand in exploring the questions described in this note and there advice during its writing. I also thank Persi Diaconis, Amir Dembo, and George Papanicolaou for useful discussions when I first worked to understand the remaining needed estimates during my early days at Stanford. I also thank Sandra McBride and BJM for reading sections. I thank the organizers of Forges-les-Eaux for the invitation to talk and the opportunity to publish this expanded version of my lecture. The also author thanks the Institute for Advanced Study for it hospitality during the year 2002-2003 when the majority of this text was written and the NSF for its support through Grant DMS-9971087.

\section{A. Comparison of Measures on Path Space}

Suppose that we have stochastic processes $X^{(i)}(t), i=1,2$ on the path space $C([0, T], \mathbb{X})$ where $\mathbb{X}$ is some Hilbert space and $T \in(0, \infty]$. Furthermore, assume that $X^{(i)}$ satisfies the equation

$$
\begin{aligned}
d X^{(i)}(t) & =f_{i}\left(t, X_{[0, t]}^{(i)}\right) d t+g d W(t), t \in[0, T] \\
X^{(i)}(0) & =x_{0} .
\end{aligned}
$$


Here, for fixed $t$ the functions $f_{1}$ and $f_{2}$ map the space $C_{[0, t]}=C([0, t], \mathbb{X})$ to $\mathbb{X}$. By $X_{[0, t]}$ we mean the segment of the trajectory on $[0, t] . W(t)$ is a cylindrical Brownian motion over a Hilbert space $\mathbb{Y}$ and $g$ is an invertible Hilbert-Schmidt operator from $\mathbb{Y} \rightarrow \mathbb{X}$. For any $\mathcal{B} \subset C_{[0, T]}$, define measures $P_{[0, T]}^{(i)}(\cdot ; \mathcal{B})$ on the path space as:

$$
P_{[0, T]}^{(i)}(A ; \mathcal{B})=P\left\{X_{[0, T]}^{(i)} \in A \cap \mathcal{B}\right\} \text {, for } A \subset C_{[0, T]}
$$

Define also $D(t, \cdot)=f_{1}(t, \cdot)-f_{2}(t, \cdot)$.

In this setting, we have the following result which is a variation on Lemma B.1 from [Mat02c] and follows quickly from Girsanov's Theorem. Similar versions of this lemma can be found in [MS03] and [BM03].

Lemma A.1. Assume there exists a constant $D_{*} \in(0, \infty)$ such that

$$
\exp \left\{\frac{1}{2} \int_{0}^{T}\left|g^{-1} D\left(t, X_{[0, t]}^{(i)}\right)\right|_{\mathbb{Y}}^{2} d t\right\} \mathbf{1}_{\mathcal{B}}\left(X_{[0, t]}^{(i)}\right)<D_{*}
$$

almost surely for $i=1,2$. Then the measures $P_{[0, T]}^{(1)}(\cdot ; \mathcal{B})$ and $P_{[0, T]}^{(2)}(\cdot ; \mathcal{B})$ are equivalent. In addition for any $p>0$

$$
\mathbb{E}\left[\frac{d P_{[0, T]}^{(1)}(\cdot ; \mathcal{B})}{d P_{[0, T]}^{(2)}(\cdot ; \mathcal{B})}\right]^{p} \leq D_{*}^{p(p-1)}
$$

And lastly

$$
\frac{1}{2}\left\|P_{[0, T]}^{(1)}(\cdot ; \mathcal{B})-P_{[0, T]}^{(2)}(\cdot ; \mathcal{B})\right\|_{T V} \leq\left(\mathbb{E}\left[\frac{d P_{[0, T]}^{(1)}(\cdot)}{d P_{[0, T]}^{(2)}(\cdot)}-1\right]^{2} \mathbf{1}_{\mathcal{B}}\right)^{\frac{1}{2}} \leq\left(D_{*}^{2}-1\right)^{\frac{1}{2}}
$$

PRoOF: Define the auxiliary SDEs

$$
d Y^{(i)}(t)=f_{i}\left(t, Y_{[0, t]}^{(i)}\right) \mathbf{1}_{\mathcal{B}(t)}\left(Y_{[0, t]}^{(i)}\right) d t+g d W(t)
$$

where $\mathcal{B}(t)=\left\{x \in C_{[0, t]}: \exists \bar{x} \in \mathcal{B}\right.$ such that $x(s)=\bar{x}(s)$ for $\left.s \in[0, t]\right\}$. Solutions $Y^{(i)}(t)$ to these equation can be constructed as

$$
Y^{(i)}(t)=X_{i}(t) \mathbf{1}_{\{t \leq \tau\}}+\left[g W(t)-g W(\tau)+X^{(i)}(\tau)\right] \mathbf{1}_{\{t>\tau\}} .
$$

Here $\tau=\inf \left\{s>0: X_{[0, s]}^{(i)} \notin \mathcal{B}(s)\right\}$.

Denote $D_{\mathcal{B}}(t, x)=\left[f_{1}(t, x)-f_{2}(t, x)\right] \mathbf{1}_{\mathcal{B}(t)}(x)$. The assumption on $D$ in (40) and the definition of $\mathcal{B}(t)$ imply that

$$
\exp \left\{\frac{1}{2} \int_{0}^{T}\left|g^{-1} D_{\mathcal{B}}\left(t, X_{[0, t]}\right)\right|_{\mathbb{Y}}^{2} d t\right\}<D_{*} \quad \text { a.s. }
$$

under both measures $P_{Y[0, t]}^{(i)}$ defining solutions to auxiliary equation with $i=1$ and $i=2$. Hence, Novikov's condition is satisfied for the difference of the drifts of 
the auxiliary equations and the Girsanov's theorem implies that $\frac{d P_{Y[0, t]}^{(1)}}{d P_{Y[0, t]}^{(2)}}(x)=\mathcal{E}(x)$ where the Radon-Nikodym derivative evaluated at a trajectory $x$ is defined by the stochastic exponent:

$$
\mathbf{E}(x)=\exp \left\{\int_{0}^{T}\left\langle g^{-1} D_{\mathcal{B}}\left(s, x_{[0, s]}\right), d W(s)\right\rangle_{\mathbb{Y}}-\frac{1}{2} \int_{0}^{T}\left|g^{-1} D_{\mathcal{B}}\left(s, x_{[0, s]}\right)\right|_{\mathbb{Y}}^{2} d s\right\} .
$$

Note that restrictions of measures $P_{Y_{[0, t]}^{(i)}}^{(i)}$ on the set $\mathcal{B}$ coincide with $P_{[0, t]}^{(i)}(\cdot ; \mathcal{B})$. This proves that $P_{[0, t]}^{(1)}(\cdot, \mathcal{B})$ is absolutely continuous with respect to $P_{[0, t]}^{(2)}(\cdot ; \mathcal{B})$. The reverse relation follows by symmetry and the proof of equivalence is complete.

To prove the second estimate, notice that

$$
\begin{aligned}
(\mathbf{E})^{p} & =\exp \left\{p \int_{0}^{T}\left\langle g^{-1} D_{\mathcal{B}}\left(s, x_{[0, s]}\right), d W(s)\right\rangle_{\mathbb{Y}}-p \frac{1}{2} \int_{0}^{T}\left|g^{-1} D_{\mathcal{B}}\left(s, x_{[0, s]}\right)\right|_{\mathbb{Y}}^{2} d s\right\} \\
& =\mathbf{E}_{p} \exp \left(\frac{p^{2}-p}{2} \int_{0}^{T}\left|g^{-1} D_{\mathcal{B}}\left(s, x_{[0, s]}\right)\right|_{\mathbb{Y}}^{2} d s\right) \leq \mathbf{E}_{p} D_{*}^{p(p-1)}
\end{aligned}
$$

where $\mathbf{E}_{p}$ is the martingale defined by

$$
\mathbf{E}_{p}=\exp \left\{p \int_{0}^{T}\left\langle g^{-1} D_{\mathcal{B}}\left(s, x_{[0, s]}\right), d W(s)\right\rangle_{\mathbb{Y}}-\frac{p^{2}}{2} \int_{0}^{T}\left|g^{-1} D_{\mathcal{B}}\left(s, x_{[0, s]}\right)\right|_{\mathbb{Y}}^{2} d s\right\} .
$$

Hence, $\mathbb{E} \mathbf{E}_{p}=1$ and in light of the estimate on $\mathbf{E}^{p}$, the proof is complete. To see the last estimate, use the Cauchy-Schwartz inequality to obtain the first inequality. The expand the square and use the fact that the Radon-Nikodym derivative is a martingale with expectation one to obtain the bound $\left(\mathbb{E}\left(\frac{d P^{(1)}}{d P^{(2)}}\right)^{2}-1\right)^{\frac{1}{2}}$. Applying the previous estimate to the square gives the result.

\section{B. Coupling Estimates}

For any two probability measure $\mu_{1}$ and $\mu_{2}$ on a space $\mathbb{X}$, we can always write them relative to a common measure $\nu$ so that $d \mu_{i}=\psi_{i} d \nu$. Then we define the measures $\left(\mu_{1} \wedge \mu_{2}\right)(\cdot),\left(\mu_{1}-\mu_{2}\right)^{+}(\cdot)$, and $\left(\mu_{2}-\mu_{1}\right)^{+}(\cdot)$ respectively by the densities $\left(\psi_{1} \wedge \psi_{2}\right) d \nu,\left(\psi_{1}-\psi_{2}\right)^{+} d \nu,\left(\psi_{2}-\psi_{1}\right)^{+} d \nu$ where $a \wedge b=\min (a, b)$ and $(a)^{+}$ is $a$ if $a$ is positive and zero otherwise. Notice that $\mu_{1}=\left(\mu_{1} \wedge \mu_{2}\right)+\left(\mu_{1}-\mu_{2}\right)^{+}$. Also observe that if $\|\cdot\|_{T V}=\int\left|\psi_{1}-\psi_{2}\right| d \nu$ is the total variation norm then $\frac{1}{2}\left\|\mu_{1}-\mu_{2}\right\|_{T V}=1-\left(\mu_{1} \wedge \mu_{2}\right)(\mathbb{X})=\left(\mu_{1}-\mu_{2}\right)^{+}(\mathbb{X})=\left(\mu_{2}-\mu_{1}\right)^{+}(\mathbb{X})$. The proof of the following lemma can be found in the appendix of [Mat02c].

Lemma B.1. Let $\mu_{1}$ and $\mu_{2}$ be two measures on a space $\mathbb{X}$ with $\mu_{i}(\mathbb{X}) \leq 1$. Assume that $\mu_{1}$ is equivalent to $\mu_{2}$ and that there exists a constant $C^{\prime}>0$ and $p>1$ so that

$$
\int_{\mathbb{X}}\left[\frac{d \mu_{1}}{d \mu_{2}}(x)\right]^{p+1} d \mu_{2}(x)=\int_{\mathbb{X}}\left[\frac{d \mu_{1}}{d \mu_{2}}(x)\right]^{p} d \mu_{1}(x)<C^{\prime}
$$


then

$$
\int_{\mathbb{X}}\left|1 \wedge \frac{d \mu_{1}}{d \mu_{2}}(x)\right| d \mu_{2}(x) \geq\left[1-\frac{1}{p}\right]\left(\frac{\mu_{1}(\mathbb{X})^{p}}{p C^{\prime}}\right)^{\frac{1}{p-1}}
$$

Notice that this lower bound is strictly positive if $\mu_{1}(\mathbb{X})>0$ (or equivalently $\mu_{2}(\mathbb{X})>$ $0)$.

\section{References}

[Arn98] Ludwig Arnold. Random dynamical systems. Springer-Verlag, Berlin, 1998.

[AS03] Andrei Acrachev and Andrey Sarychev. Navier-stokes equation controlled by degenerate forcing: Controllabillity in finite-dimentional projections. Preprint, 2003.

[Bak02] Yu. Yu. Bakhtin. Existence and uniqueness of stationary solution of stochastic differential equation with memory. Theory Probab. Appl, 47(4):764-769, 2002.

[Bax91] Peter H. Baxendale. Statistical equilibrium and two-point motion for a stochastic flow of diffeomorphisms. In Spatial stochastic processes, volume 19 of Progress in Probability, pages 189-218. Birkhäuser Boston, Boston, MA, 1991.

[Bel95] Denis R. Bell. Degenerate stochastic differential equations and hypoellipticity. Longman, Harlow, 1995.

[BKL00] J. Bricmont, A. Kupiainen, and R. Lefevere. Probabilistic estimates for the two-dimensional stochastic Navier-Stokes equations. J. Statist. Phys., 100(3-4):743-756, 2000.

[BKL01] J. Bricmont, A. Kupiainen, and R. Lefevere. Ergodicity of the 2D NavierStokes equations with random forcing. Comm. Math. Phys., 224(1):6581, 2001. Dedicated to Joel L. Lebowitz.

[BKL02] J. Bricmont, A. Kupiainen, and R. Lefevere. Exponential mixing of the 2D stochastic Navier-Stokes dynamics. Comm. Math. Phys., 230(1):87$132,2002$.

[BM03] Yuri Bakhtin and Jonathan C. Mattingly. Stationary solutions of stochastic differential equation with memory and stochastic partial differential equations. Preprint, 2003.

[CDF97] Hans Crauel, Arnaud Debussche, and Franco Flandoli. Random attractors. J. Dynam. Differential Equations, 9(2):307-341, 1997. 
[Cer99] Sandra Cerrai. Ergodicity for stochastic reaction-diffusion systems with polynomial coefficients. Stochastics Stochastics Rep., 67(1-2):17-51, 1999.

[CF88] Peter Constantin and Ciprian Foiaș. Navier-Stokes Equations. University of Chicago Press, Chicago, 1988.

[CFNT89] P. Constantin, C. Foias, B. Nicolaenko, and R. Temam. Integral manifolds and inertial manifolds for dissipative partial differential equations, volume 70 of Applied Mathematical Sciences. Springer-Verlag, New YorkBerlin, 1989.

[CFS82] I. P. Cornfeld, S. V. Fomin, and Ya. G. Sinaŭ. Ergodic theory, volume 245 of Grundlehren der Mathematischen Wissenschaften. Springer-Verlag, New York-Berlin, 1982.

[CK97] Pao-Liu Chow and Rafail Z. Khasminskii. Stationary solutions of nonlinear stochastic evolution equations. Stochastic Anal. Appl., 15(5):671699, 1997.

[DG95] Charles R. Doering and J. D. Gibbon. Applied analysis of the NavierStokes equations. Cambridge Texts in Applied Mathematics. Cambridge University Press, Cambridge, 1995.

[DLJ88] R. W. R. Darling and Yves Le Jan. The statistical equilibrium of an isotropic stochastic flow with negative Lyapounov exponents is trivial. In Séminaire de Probabilités, XXII, volume 1321 of Lecture Notes in Math., pages 175-185. Springer, Berlin, 1988.

[DPZ92] Giuseppe Da Prato and Jerzy Zabczyk. Stochastic Equations in Infinite Dimensions. Cambridge, 1992.

[DPZ96] Giuseppe Da Prato and Jerzy Zabczyk. Ergodicity for Infinite Dimensional Systems. Cambridge, 1996.

[DPZ02] Giuseppe Da Prato and Jerzy Zabczyk. Second order partial differential equations in Hilbert spaces, volume 293 of London Mathematical Society Lecture Note Series. Cambridge University Press, Cambridge, 2002.

[DT95] Charles R. Doering and Edriss S. Titi. Exponential decay rate of the power spectrum for solutions of the Navier-Stokes equations. Phys. Fluids, 7(6):1384-1390, 1995.

[Dud76] R. M. Dudley. Probabilities and metrics. Matematisk Institut, Aarhus Universitet, Aarhus, 1976. Convergence of laws on metric spaces, with a view to statistical testing, Lecture Notes Series, No. 45.

[EFNT94] A. Eden, C. Foias, B Nicolaenko, and R. Temam. Exponential Attractors for dissipative Evolution equations. Research in Applied Mathematics. John Wiley and Sons and Masson, 1994. 
[EH01] J.-P. Eckmann and M. Hairer. Uniqueness of the invariant measure for a stochastic PDE driven by degenerate noise. Comm. Math. Phys., 219(3):523-565, 2001.

[EKMS00] Weinan E, K. Khanin, A. Mazel, and Ya. Sinai. Invariant measures for Burgers equation with stochastic forcing. Ann. of Math. (2), 151(3):877960, 2000.

[EL02] Weinan E and Di Liu. Gibbsian dynamics and invariant measures for stochastic dissipative PDEs. J. Statist. Phys., 108(5/6):1125-1156, 2002.

[EM01] Weinan E and Jonathan C. Mattingly. Ergodicity for the Navier-Stokes equation with degenerate random forcing: finite-dimensional approximation. Comm. Pure Appl. Math., 54(11):1386-1402, 2001.

[EMS01] Weinan E, J. C. Mattingly, and Ya G. Sinai. Gibbsian dynamics and ergodicity for the stochastic forced navier-stokes equation. Comm. Math. Phys., 224(1), 2001.

[EVE00] Weinan E and Eric Vanden Eijnden. Generalized flows, intrinsic stochasticity, and turbulent transport. Proc. Natl. Acad. Sci. USA, 97(15):82008205 (electronic), 2000.

[Fer97] Benedetta Ferrario. Ergodic results for stochastic Navier-Stokes equation. Stochastics and Stochastics Reports, 60(3-4):271-288, 1997.

[FG98] F. Flandoli and F. Gozzi. Kolmogorov equation associated to a stochastic Navier-Stokes equation. J. Funct. Anal., 160(1):312-336, 1998.

[Fla94] Franco Flandoli. Dissipativity and invariant measures for stochastic Navier-Stokes equations. NoDEA, 1:403-426, 1994.

[FM95] Franco Flandoli and B. Maslowski. Ergodicity of the 2-D Navier-Stokes equation under random perturbations. Comm. in Math. Phys., 171:119$141,1995$.

[FP67] C. Foiaș and G. Prodi. Sur le comportement global des solutions nonstationnaires des équations de Navier-Stokes en dimension 2. Rend. Sem. Mat. Univ. Padova, 39:1-34, 1967.

[FST88] Ciprian Foias, George R. Sell, and Roger Temam. Inertial manifolds for nonlinear evolutionary equations. J. Differential Equations, 73(2):309353, 1988.

[FT89] C. Foias and R. Temam. Gevrey class regularity for the solutions of the Navier-Stokes equations. J. Funct. Anal., 87(2):359-369, 1989.

[Hai02] M. Hairer. Exponential mixing properties of stochastic PDEs through asymptotic coupling. Probab. Theory Related Fields, 124(3):345-380, 2002 . 
[Jur97] Velimir Jurdjevic. Geometric control theory, volume 52 of Cambridge Studies in Advanced Mathematics. Cambridge University Press, Cambridge, 1997.

[Kif86] Yuri Kifer. Ergodic theory of random transformations. Birkhäuser Boston Inc., Boston, MA, 1986.

[KPS02] Sergei Kuksin, Andrey Piatnitski, and Armen Shirikyan. A coupling approach to randomly forced nonlinear PDEs. II. Comm. Math. Phys., 230(1):81-85, 2002.

[KS00] Sergei Kuksin and Armen Shirikyan. Stochastic dissipative PDEs and Gibbs measures. Comm. Math. Phys., 213(2):291-330, 2000.

[KS02] Sergei Kuksin and Armen Shirikyan. Coupling approach to white-forced nonlinear PDEs. J. Math. Pures Appl. (9), 81(6):567-602, 2002.

[Kuk03] Sergei Kuksin. Eulerian limit for 2d statistical hydrodynamics. Preprint, 2003.

[KS84] Shigeo Kusuoka and Daniel Stroock. Applications of the Malliavin calculus. I. In Stochastic analysis (Katata/Kyoto, 1982), pages 271-306. North-Holland, Amsterdam, 1984.

[LJ87] Y. Le Jan. Équilibre statistique pour les produits de difféomorphismes aléatoires indépendants. Ann. Inst. H. Poincaré Probab. Statist., 23(1):111-120, 1987.

[LO97] C. David Levermore and Marcel Oliver. Analyticity of solutions for a generalized Euler equation. J. Differential Equations, 133(2):321-339, 1997.

[Mat98] Jonathan C. Mattingly. The Stochastically forced Navier-Stokes equations: energy estimates and phase space contraction. PhD thesis, Princeton University, 1998.

[Mat99] Jonathan C. Mattingly. Ergodicity of 2D Navier-Stokes equations with random forcing and large viscosity. Comm. Math. Phys., 206(2):273-288, 1999.

[Mat02a] Jonathan C. Mattingly. Contractivity and ergodicity of the random map $x \mapsto|x-\theta|$. Theory of Probability and its Applications, 47(2):388-397, 2002 .

[Mat02b] Jonathan C. Mattingly. The dissipative scale of the stochastics NavierStokes equation: regularization and analyticity. J. Statist. Phys., 108(56):1157-1179, 2002.

[Mat02c] Jonathan C. Mattingly. Exponential convergence for the stochastically forced Navier-Stokes equations and other partially dissipative dynamics. Comm. Math. Phys., 230(3):421-462, 2002. 
[MP03] Jonathan C. Mattingly and Étienne Pardoux. Malliavin calculus and the randomly forced Navier Stokes equation. Preprint, 2003.

[MR] R. Mikulevicius and B. L. Rozovskii. Stochastic navier-stokes equations for turbulent flows. Preprint.

[MS99] J. C. Mattingly and Ya. G. Sinai. An elementary proof of the existence and uniqueness theorem for the Navier-Stokes equations. Commun. Contemp. Math., 1(4):497-516, 1999.

[MS03] Jonathan C. Mattingly and Toufic M. Suidan. The small scales of the stochastic navier stokes equations under rough forcing. Preprint, 2003.

[MSH02] J. C. Mattingly, A.M. Stuart, and D. J. Higham. Ergodicity for SDEs and approximations: Locally lipschitz vector fields and degenerate noise. Stochastic Process. Appl. 101, no. 2, 185-232, 2002.

[MT93] S. P. Meyn and R. L. Tweedie. Markov Chains and Stochastic Stability. Springer-Verlag, 1993.

[MY02] Nader Masmoudi and Lai-Sang Young. Ergodic theory of infinite dimensional systems with applications to dissipative parabolic PDEs. Comm. Math. Phys., 227(3):461-481, 2002.

[Nor86] James Norris. Simplified Malliavin calculus. In Séminaire de Probabilités, XX, 1984/85, pages 101-130. Springer, Berlin, 1986.

[Oks92] Bernt Oksendal. Stochastic Differential Equations. Springer-Verlag, 3nd edition, 1992.

[OT00] Marcel Oliver and Edriss S. Titi. Remark on the rate of decay of higher order derivatives for solutions to the Navier-Stokes equations in $\mathbf{r}^{n}$. J. Funct. Anal., 172(1):1-18, 2000.

[Rom02] Marco Romito. Ergodicity of the finite dimensional approximation of the 3d navier-stokes equations forced by a degenerate. Peprint, 2002.

[RY94] Daniel Revuz and Marc Yor. Continuous martingales and Brownian motion, volume 293 of Grundlehren der Mathematischen Wissenschaften. Springer-Verlag, Berlin, second edition, 1994.

[Sch97] Björn Schmalfuss. Qualitative properties for the stochastic Navier-Stokes equation. Nonlinear Anal., 28(9):1545-1563, 1997.

[Shi02] Armin Shirikyan. A version of the law of large number and applications. In Probabilistic Methods in Fluids. World Scientific, 2002.

[Sin94] Ya. G. Sină. Topics in ergodic theory, volume 44 of Princeton Mathematical Series. Princeton University Press, Princeton, NJ, 1994. 
[Tem95] Roger Temam. Navier-Stokes equations and nonlinear functional analysis, volume 66 of CBMS-NSF Regional Conference Series in Applied Mathematics. Society for Industrial and Applied Mathematics (SIAM), Philadelphia, PA, second edition, 1995.

[VF88] M.J. Vishik and A.V. Fursikov. Mathematical Problems of Statistical Hydrodynamics. Kluwer Academic Publishers, 1988. Updated version of Russian original of same name.

School of Mathematics, Institute For Advanced Study, PRINCETON NJ, USA

and Department of Mathematics, Duke University, DURHAM NC, USA

jonm@math . duke. edu

www.math.duke.edu $/ \sim$ jonm 